\title{
The structure of pseudo-holomorphic subvarieties for a degenerate almost complex structure and symplectic form on $S^{1} \times B^{3}$
}

\author{
Clifford Henry Taubes \\ Department of Mathematics \\ Harvard University \\ Cambridge, MA 02138, USA
}

Email: chtaubes@abel.math.harvard.edu

\begin{abstract}
A self-dual harmonic 2-form on a 4-dimensional Riemannian manifold is symplectic where it does not vanish. Furthermore, away from the form's zero set, the metric and the 2-form give a compatible almost complex structure and thus pseudo-holomorphic subvarieties. Such a subvariety is said to have finite energy when the integral over the variety of the given self-dual 2-form is finite. This article proves a regularity theorem for such finite energy subvarieties when the metric is particularly simple near the form's zero set. To be more precise, this article's main result asserts the following: Assume that the zero set of the form is non-degenerate and that the metric near the zero set has a certain canonical form. Then, except possibly for a finite set of points on the zero set, each point on the zero set has a ball neighborhood which intersects the subvariety as a finite set of components, and the closure of each component is a real analytically embedded half disk whose boundary coincides with the zero set of the form.
\end{abstract}

\section{AMS Classification numbers Primary: 53C07}

Secondary: $52 \mathrm{C} 15$

Keywords: Four-manifold invariants, symplectic geometry

Proposed: Robion Kirby

Seconded: Gang Tian, Tomasz Mrowka
Received: 2 February 1998

Revised: 20 November 1998 


\section{Introduction}

The purpose of this paper is to prove a regularity theorem for pseudo-holomorphic curves on the 4 -manifold $X=S^{1} \times B^{3}$ with a degenerate symplectic structure. Here, $S^{1}$ is the circle and $B^{3}$ is the standard 3-ball in $\mathbb{R}^{3}$. Let $t$ be the standard coordinate on the circle, and let $(x, y, z)$ be Euclidean coordinates on $\mathbb{R}^{3}$. Consider now the 2 -form:

$$
\omega=d t \wedge(x d x+y d y-2 z d z)+x d y \wedge d z-y d x \wedge d z-2 z d x \wedge d y
$$

One can check that this form is closed; moreover, $\omega$ is self-dual with respect to the obvious product flat metric on $S^{1} \times B^{3}$. Thus, $\omega$ is non-degenerate where $\omega$ is non-zero. Indeed,

$$
\omega \wedge \omega=2 \cdot\left(x^{2}+y^{2}+4 \cdot z^{2}\right) \cdot d t \wedge d x \wedge d y \wedge d z
$$

and so $\omega$ vanishes only on the circle $Z=S^{1} \times\{0\}$.

On the complement of the circle $Z$, the form $\omega$ is non-degenerate and so symplectic. On this complement, $\omega$ and the flat, product metric define an almost complex structure, $J$, which is given as follows: First, introduce

$$
g=\left(x^{2}+y^{2}+4 \cdot z^{2}\right)^{\frac{1}{2}}
$$

which is the norm of $\omega$.

Then, set:

- $J \cdot \partial_{t}=-g^{-1}\left(x \cdot \partial_{x}+y \cdot \partial_{y}-2 \cdot z \cdot \partial_{z}\right)$

- $J \cdot \partial_{x}=g^{-1}\left(x \cdot \partial_{t}+y \cdot \partial_{z}+2 \cdot z \cdot \partial_{y}\right)$

- $J \cdot \partial_{y}=g^{-1}\left(y \cdot \partial_{t}-x \cdot \partial_{z}-2 \cdot z \cdot \partial_{x}\right)$

- $J \cdot \partial_{z}=g^{-1}\left(-2 \cdot z \cdot \partial_{t}+x \cdot \partial_{y}-y \cdot \partial_{x}\right)$.

By the way, note that $J$ is $\omega$-compatible as $\sqrt{2} \omega(\cdot, J(\cdot)) /|\omega|$ gives back the product, flat metric. Note as well that $J$ is singular on $Z$.

This example of the self-dual, closed form $\omega$ on the 4 -manifold $S^{1} \times B^{3}$ serves as a model for singular symplectic forms defined by closed, self-dual forms on general 4-manifolds. The relevance of the $\left(S^{1} \times B^{3}, \omega\right)$ model in this more general context is remarked upon further in subsection 1.c, below.

Now consider:

Definition 1.1 Let $Y$ be a smooth 4 -manifold (in this case, $S^{1} \times\left(B^{3}-\{0\}\right)$ with symplectic form $\omega$ and compatible almost complex structure $J$. A subset $C \subset Y$ will be called a pseudo-holomorphic subvariety if the following is true: 
- $C$ is closed.

- There exists a $C^{\infty}$ complex curve $C_{0}$ together with a proper, $J$-pseudoholomorphic map $f: C_{0} \rightarrow Y$ whose image is $C$ and which is an embedding on the complement of a countable set of points in $C_{0}$. The complex curve $C_{0}$ will be called the smooth model for $C$.

Call $C$ a pseudo-holomorphic submanifold when $C$ is non-singular.

The next definition introduces the notion of finite energy for a pseudo-holomorphic subvariety.

Definition 1.2 Let $C$ be a pseudo-holomorphic subvariety as in Definition 1.1. Say that $C$ has finite energy when the integral over $C$ of $\omega$ is finite. (This is equivalent to the assertion that the integral over the smooth model $C_{0}$ of $f^{*} \omega$ is finite.)

(Note that $\left.\omega\right|_{T C}>0$ so that there is no question here with conditional convergence of the integral.)

The main theorem in this article (Theorem 1.8, below) describes the nature of a finite energy, pseudo-holomophic subvariety near $Z$. However, part of the story is simply this:

Fix a finite energy, pseudo-holomorphic subvariety $C$. Then, all but finitely many points in $Z$ have a neighborhood in $S^{1} \times B^{3}$ in which $C$ has finitely many components. Moreover, each of these components is a smoothly embedded halfdisk (that is, the image of $\{w \in \mathbb{C}:|w|<1$ and $\operatorname{im}(w) \geq 0\}$ ). Furthermore, the boundary line (where $\operatorname{im}(w)=0$ ) of each such half-disk lies on $Z$.

\section{1.a Examples}

Before turning to the precise statement of the main theorem, some examples are in order.

The first examples below are the simplest cases of finite energy, pseudo-holomorphic submanifolds whose closure in $S^{1} \times B^{3}$ is a submanifold with boundary where the latter coincides with $Z$.

Example 1.3 Fix a point $\nu$ on the boundary of unit disk in the $(x, y)$ plane (where $z=0$ ) in $\mathbb{R}^{3}$. Let $\lambda_{v}$ denote the complement of the origin in the line segment between the origin in $\mathbb{R}^{3}$ and $\nu$. Then

$$
C=S^{1} \times \lambda_{v}
$$




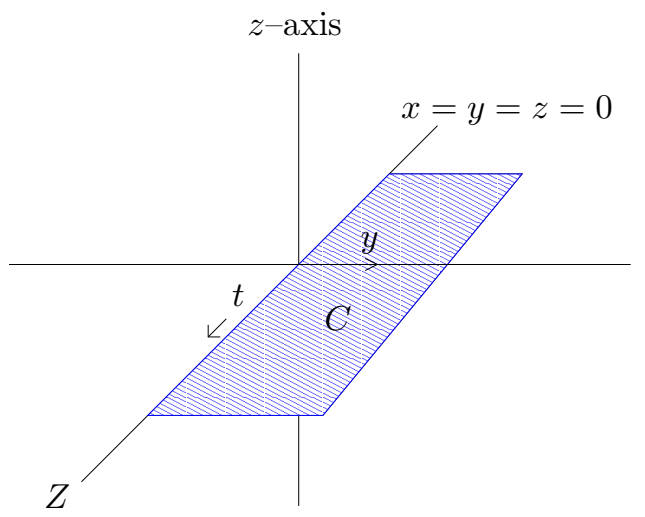

Figure 1

is a smooth, pseudo-holomorphic subvariety. See Figure 1.

Example 1.4 Let $\nu=(0,0, \pm 1) \in \mathbb{R}^{3}$ and define $\lambda_{\nu}$ as before, and then define $C$ as in (1.5). See Figure 2.

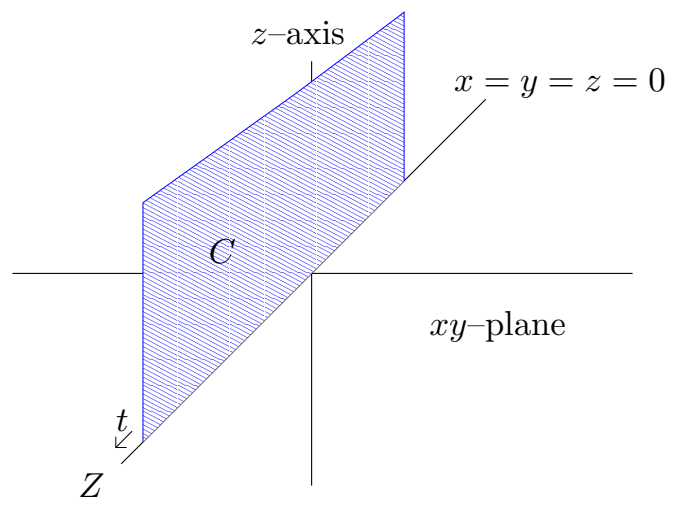

Figure 2

Note that in both of these examples, $C$ is parametrized in the obvious way by points $(t, s) \in S^{1} \times(0,1]$, and the pull-back to $C$ of the form $\omega$ is $d t \wedge s \cdot d s$ when $\eta$ lies in the $(x, y)$ plane, and the pull-back is $2 \cdot d t \wedge s \cdot d s$ when $\eta$ is on the $z$-axis. Thus, in either case, $C$ has finite energy. Indeed, the integral of $\omega$ over $C$ 's intersection with the product of $S^{1}$ and the ball of radius $r$ and center 0 in $\mathbb{R}^{3}$ is equal to $r^{2} / 2$ in the first case, and $r^{2}$ in the second. (The similarities between the cases in Example 1.3 and those from Example 1.4 are superficial, as can be seen in Theorem 1.8 below.) 
The next examples are cones. Each has closure which intersects $Z$ in a single point. For these examples, let $(\rho, \phi)$ denote the standard polar coordinates in the $(x, y)$ plane so that $x=\rho \cdot \cos \phi$ and $y=\rho \cdot \sin \phi$.

Example 1.5 Fix an origin $t_{0} \in S^{1}$. Let $c \in\left(0,2 / 3^{3 / 2}\right]$ be a constant and let $u(\cdot)$ be a positive function on $\mathbb{R}$ which satisfies the differential equation

$$
u^{\prime \prime}+\frac{4}{9} u-\frac{2}{9} c u^{-2}=0
$$

with the normalization $\frac{9}{4}\left(u^{\prime}\right)^{2}+u^{2}+c / u=1$. (Here, ' denotes differentiation.) Note that all such functions are periodic and there exist solutions with period in $2 \pi \mathbb{Q}$. Let $u$ be one of the latter and write its period as $2 \pi a / b$ where $a$ and $b$ are positive integers which are relatively prime. Introduce the pair of pseudoholomorphic submanifolds $\Sigma_{ \pm} \subset S^{1} \times B^{3}$ by using $s \in(0, \infty)$ and $\tau \in[0,2 \pi b]$ as coordinates and using the parametrization rule:

- $t=t_{0} \pm s \frac{3}{2} u^{\prime}(\tau)$

- $z= \pm s u(\tau)$

- $\rho=s c^{\frac{1}{2}}|u(\tau)|^{-\frac{1}{2}}$

- $\phi=\tau$

In the extreme case where $c=2 / 3^{3 / 2}$, the solution to (1.6) is $u \equiv 1 / 3^{1 / 2}$. The corresponding submanifold from (1.7) lies in the time slice $t=t_{0}$ and coincides with either the $z>0$ (for $\Sigma_{+}$) or the $z<0$ (for $\Sigma_{-}$) half of the cone $x^{2}+y^{2}-2 \cdot z^{2}=0$ in $B^{3}$. See Figure 3 .

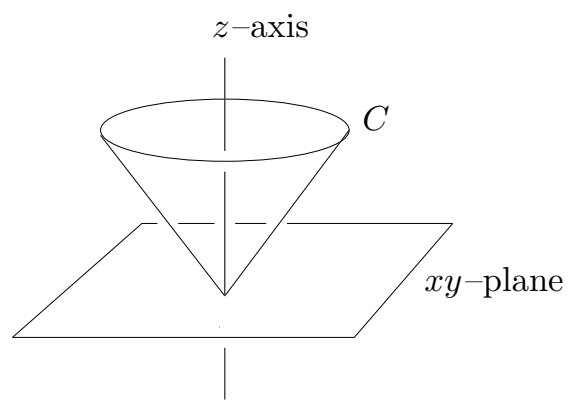

Figure 3

(Note that the cones in (1.7) are embedded because of the assumption that $a$ and $b$ are relatively prime.) The fact that $\Sigma_{ \pm}$in (1.7) is pseudo-holomorphic can be checked directly, or the reader can refer to an argument which is given in the proof of Proposition 3.2 in Section 3. The other assertions about $\Sigma_{ \pm}$ 

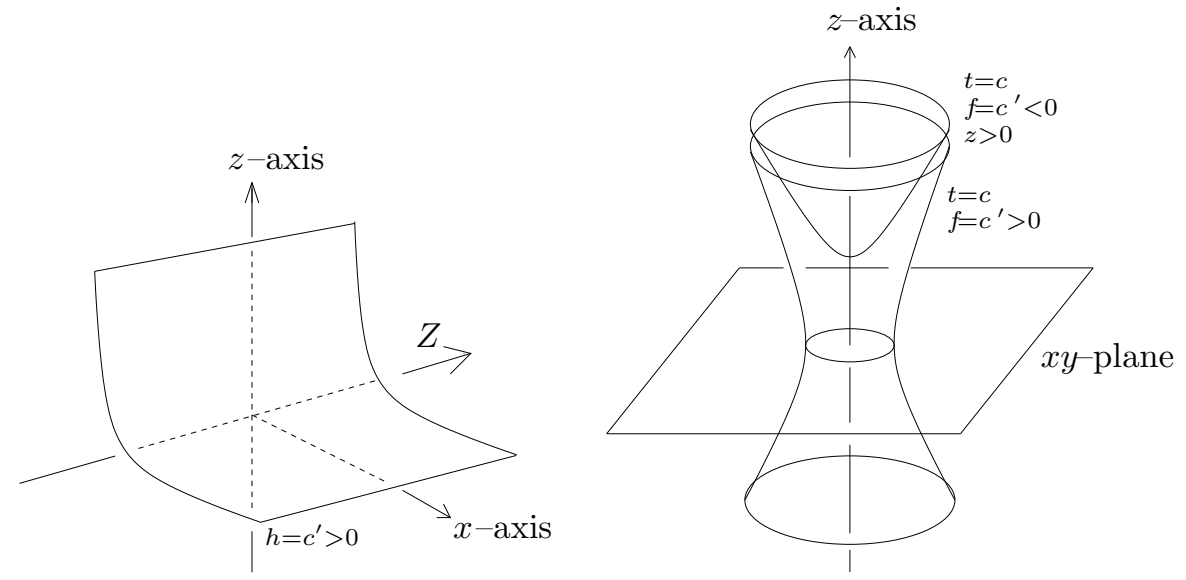

Figure 4

and the assertions about the solutions to (1.6) are proved as a part of Lemma 1.9 in subection 1.d, below.

These last examples also have finite energy. Indeed, the ball $B_{r} \subset S^{1} \times B^{3}$ of radius $r$ about the point $\left(t_{0}, 0\right)$ contains the intersection of $\Sigma_{ \pm}$with a neighborhood of $Z$; meanwhile, the fact that $\Sigma_{ \pm}$is a cone while $\omega$ has homogeneous dependence on the coordinates implies that the integral of $\omega$ over $\Sigma_{ \pm} \cap B_{r}$ is equal to $r^{3} \delta_{c}$. Here, $\delta_{c}$ is a constant which depends on $c$. Note however that there is a $c$-independent constant $\zeta \geq 1$ with the property that $\delta_{c} \geq \zeta^{-1}$. (This last assertion is restated as Lemma 1.10 in subsection 1.d.)

For the next example, introduce the following pair of functions on $B^{3}$ :

- $f=2^{-1} \cdot\left(x^{2}+y^{2}-2 \cdot z^{2}\right)$

- $h=z \cdot\left(x^{2}+y^{2}\right)$

The next example includes smooth pseudo-holomorphic subvarieties which miss an entire neighborhood of $Z$.

Example 1.6 Let $c$ and $c^{\prime}$ be constants and let $C$ denote the submanifold in $S^{1} \times(B-0)$ which is described by one of the following. See Figure 4 .

- $\phi=c$ and $h=c^{\prime}$

- $t=c$ and $f=c^{\prime}>0$

- $t=c$ and $f=c^{\prime} \leq 0$ and $\pm z>0$

Geometry $\&$ Topology, Volume 2 (1998) 
Note that the submanifolds in the first line of (1.9) whose closure in $X$ intersect $Z$ are precisely the $C$ 's from Examples 1.3 and 1.4. Likewise, those in the second line of (1.9) whose closure in $X$ intersect $Z$ are precisely the $C$ 's from the extreme case $c=2 / 3^{3 / 2}$ in Example 1.5.

By the way, as the constants $c$ and $c^{\prime}$ vary in (1.9) and Figure 4, the pseudoholomorphic subvarieties provide a pair of mutually orthogonal, 2-dimensional foliations of $S^{1} \times\left(B^{3}-0\right)$ by finite energy, pseudoholomorphic subvarieties. Indeed, the existence of such a pair of foliations is related to a very useful fact: the form $\omega$ and the flat metric are particularly nice in the $(t, f, h, \phi)$ coordinates. Indeed,

$$
\omega=d t \wedge d f+d \phi \wedge d h
$$

while the flat metric is

$$
d t^{2}+g^{-2} \cdot d f^{2}+g^{-2} \cdot \rho^{-2} \cdot d h^{2}+\rho^{2} \cdot d \phi^{2} .
$$

(The fact that (1.9) are pseudo-holomorphic follows immediately from (1.10) and (1.11).)

The final example, below, gives additional cases of a pseudo-holomorphic subvariety whose closure in some neighborhood of each point in $Z$ is a union of embedded half-disks. Note that these examples are connected, but even so, some do not have submanifold with boundary closures in any open set which contains all of $Z$.

Example 1.7 Let $q>0$ and $p$ be relatively prime integers and let $\alpha \in S^{1}$. Let $C$ denote the image of the map from $S^{1} \times(0,1]$ into $S^{1} \times(B-0)$ which sends $(\phi, s)$ to

$$
(q \cdot \phi, s \cdot \cos (p \cdot \phi+\alpha), s \cdot \sin (p \cdot \phi+\alpha), z(s)) .
$$

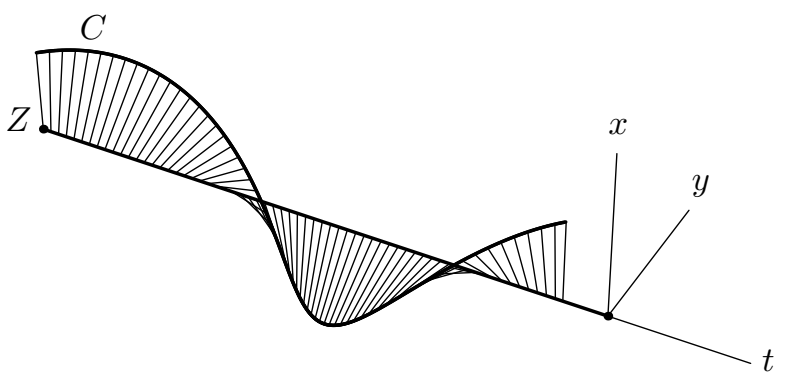

Figure 5

See Figure 5 . Here, $z(\cdot)$ is the smooth function on $(0,1]$ which obeys: 
- $\quad(q+2 p \cdot z) \cdot s \cdot z^{\prime}+2 q \cdot z=p \cdot s^{2}$

- $\quad z(s)=\frac{p}{4 q} \cdot s^{2}+\mathcal{O}\left(s^{4}\right)$

Note that in the case $p=0$, this $C$ is the same as in Example 1.3.

\section{1.b The main theorem}

The following theorem summarizes the principle results of this article:

Theorem 1.8 Let $C \subset S^{1} \times\left(B^{3}=\{0\}\right)$ be a finite energy, pseudo-holomorphic subvariety. Then $C$ has finite area and each point in its closure in $S^{1} \times B^{3}$ has well defined tangent cones up to the rotation $\phi \rightarrow \phi+$ constant. Furthermore, there is a finite set $Z_{s} \subset Z=S^{1} \times\{0\}$ and a positive integer $N$ with the following significance: Let $\tau \in Z-Z_{s}$. Then $\tau$ centers an open ball in $S^{1} \times B^{3}$ whose intersection with $C$ is a disjoint union of $N$ embedded components. Furthermore, the closure of each such component is the image of the standard half disk via a real analytic embedding which sends the straight edge to $Z$ and the interior to the complement of $Z$. Finally, this embedding is described by one of the following two possibilities:

- The image is the half disk $\left\{(t, x, y, z):(t-\tau)^{2}+z^{2} \leq \varepsilon^{2}, x=y=0\right.$ and either $z \geq 0$ or $z \leq 0\}$. This is a half disk in the $x=y=0$ plane and a part of the submanifold in Example 1.4.

- The image is that of the half disk $\left\{(t, \rho) \in \mathbb{R}^{2}:(t-\tau)^{2}+\rho^{2} \leq \varepsilon^{2}\right.$ and $\left.\rho \geq 0\right\}$. The embedding of this half disk is defined by functions $(\phi=\phi(t, \rho), z=z(t, \rho)\}$ via the parametrization $(x=\rho \cos \phi, y=\rho \sin \phi, z)$. Here, $(\rho, z)$ are real analytic functions of $(t, \rho)$ which obey $\phi(t, \rho)=\phi_{0}(t)+\mathcal{O}\left(\rho^{2}\right)$ and $z(t, \rho)=$ $4^{-1} \phi_{0}^{\prime}(t) \cdot \rho^{2}+\mathcal{O}\left(\rho^{4}\right)$ near $\rho=0$. In particular, this portion of $C$ is, locally, a perturbation of an Example 1.3 model. (See also Example 1.7.)

The set $Z_{s} \subset Z$ will be called the singular set. The behavior of $C$ near $Z_{s}$ can also be described in some detail. The simplest sort of singularity is given in Example 1.5, and multi-sheeted versions of Example 1.5 can also occur. However, there is also a second, less concentrated type of singularity which can be viewed as a perturbation of the $h=$ constant $(\neq 0)$ and $\phi=$ constant example from Example 1.6. In the latter, $\phi$ and $h$ become (slowly varying) functions of $t$ and $f$ where $h$ vanishes at a point where $f=0$ and $t=\tau \in Z_{s}$. Some of the structure of $C$ near $Z_{s}$ is elucidated in Proposition 4.1, below. In any event, the detailed structure of $C$ near $Z_{s}$ and the question of eliminating $Z_{s}$ by perturbations will be the subject of a planned sequel to this article. 
As a parenthetical remark, note that Hofer, Wysocki and Zehnder in [6], [7] have studied (using different techniques) pseudo-holomorphic curves on $M^{3} \times \mathbb{R}$ in the case when the symplectic form arises from a contact structure on $M^{3}$. See also [8] for a more recent application of this approach.

The rest of this article is more or less centered around the proof of Theorem 1.8, although various constructions are presented with regard to pseudoholomorphic subvarieties in $S^{1} \times\left(B^{3}-\{0\}\right)$ which may have some broader utility. Aside from subsections 1.c and 1.d, below, there are Sections 2-9 plus a lengthy appendix. With regard to the proof of Theorem 1.8, the reader should note that the assertions of the theorem are either reasserted as parts of propositions below or are their direct corollaries. In particular, the assertion that $C$ has finite area is part of Proposition 3.2. The fact that the tangent cones are unique up to rotation follows from Proposition 4.1. The assertion that there are at most finitely many singular points on $Z$ follows from Propositions 5.1 and 9.1. Finally, the description of $C$ near a regular point in $Z$ comes from Proposition 7.1.

In any event, here is a table of contents for this article:

Section 1 Introduction: Definitions, examples and the main theorem

Section 2 Finite energy and finite local energy: The derivation of a key monotonicity formula for the local energy in a ball centered on $Z$

Section 3 Limits: The ultra-local structure near $Z$ as studied via successive dilations and their limits

Section 4 The multiplicity of the limit: A uniqueness theorem for dilation limits

Section 5 The integers $n_{ \pm}$: A proof that there are only finitely many singularities like Example 1.5

Section 6 The semi-continuity of $p$ and $q$ as functions on $Z$ : How local sheet number can change

Section 7 Regular points: The discussion of the first bullet in Theorem 1.8

Section 8 A proof that $C_{\rho}$ is smooth: The discussion of the second bullet in Theorem 1.8

Section 9 The number of singular points: The proof that there are only finitely many

Appendix: Proofs of some assertions about multi-sheeted examples 
Before proceeding to the subsequent subsections of Section 1 or to Section 2, please be advised of three conventions that are used in the subsequent sections of this article. First, many equations involve constants whose precise value is not terribly relevant to the discussion. Such constants are typically denoted below by $\zeta$. Thus, the value of $\zeta$ will change from appearance to appearance.

The second convention involves metrics on a pseudo-holomorphic subvariety $C$ : The convention here is that the metric at the smooth points of $C$ is that which is induced by $C$ 's embedding into $S^{1} \times B^{3}$.

The third convention concerns the notation for the restriction to $C$ of a differential form from $S^{1} \times B^{3}$ : No special notation will be used. That is, the restricted form and the original form will not be notationally distinguished. The context will usually make things clear.

With regard to the previous two conventions, the reader should take special care to note that the norm on $C$ of a restricted form may differ from its norm in $S^{1} \times B^{3}$ although both norms will be written alike. (Note that the former norm is never greater than the latter.)

\section{1.c A comment about a Riemannian generalizaton}

This subsection constitutes a digression of sorts in order to provide the promised brief remarks concerning the relevance of Theorem 1.8 in the wider context of degenerate symplectic forms on 4-manifolds which come from closed, self-dual 2 -forms. (See also [18].)

To start the digression, remark that if $X$ is a compact, oriented, Riemannian 4-manifold, then there are non-trivial, harmonic self-dual 2-forms if the intersection form on $H_{2}(X ; \mathbb{Z})$ is not negative definite. (Celebrated theorems of Donaldson [2] and Freedman [4] assert that a smooth, simply connected 4manifolds with negative definite intersection form is homeomorphic to the multiple connect sum of $S^{4}$ with some number of $\mathbb{C P}^{2}$ 's having the non-complex orientation.)

With the preceding understood, let $X$ be a compact, oriented, 4-manifold whose intersection form is not negative definite and let $\omega_{X}$ be a non-trivial, closed and self-dual 2-form on $X$. As $\omega_{X} \wedge \omega_{X}=\left|\omega_{X}\right|^{2} d$ vol, this form is symplectic where it does not vanish. For some time, a number of geometers have been aware (see, eg [19] and [3]) of the fact that when the metric on $\mathrm{X}$ is suitably generic, then there is a closed, self-dual 2 -form $\omega_{X}$ whose zero set is cut out transversely as a section of the self-dual 3-plane subbundle of $\Lambda^{2} T^{*} X$. 
(See, eg [11] or [9] for a published proof of this fact.) When such is the case, then $\omega_{X}^{-1}(0)$ is a union of embedded circles in $X$. Furthermore, a tubular neighborhood of such a circle can be parametrized as $S^{1} \times B^{3}$ with coordinates $t \in S^{1}=\mathbb{R} / \mathbb{Z}$ and $\left(x_{1}, x_{2}, x_{3}\right) \in B^{3}$ so that $\omega_{X}$ has the form

$$
\omega_{x}=d t \wedge A_{i j} x^{i} d x^{j}+A_{i j} x^{i} \varepsilon^{j k m} d x^{k} \wedge d x^{m}+\mathcal{O}\left(|x|^{2}\right)
$$

where $\left\{A_{i j}=A_{i j}(t)\right\}_{i, j=1, \ldots, 3}$ are the components of a $t$-dependent, symmetric, trace zero and non-degenerate $3 \times 3$ matrix. (In (1.14), repeated indices are summed. Also, $\left\{\varepsilon^{j k m}\right\}$ are the components of the completely anti-symmetric, $3 \times 3$ matrix with $\varepsilon^{123}=1$.) Note that by changing the orientation of $S^{1}$ if necessary, one can assume that $A_{i j}(t)$ has, at each $t$, precisely one negative eigenvalue and two positive eigenvalues.

After passing to a double cover of $S^{1} \times B^{3}$ (if necessary), one can find a further change of coordinates (of the form $x_{j} \rightarrow B_{j k} x_{k}$, where $\left(B_{j k}\right)$ is a $t$-dependent, invertible matrix) so that in these new coordinates, $\omega_{X}=\omega+\mathcal{O}\left(\Sigma_{j}\left|x_{j}\right|^{2}\right)$, where $\omega$ is as presented in (1.2). Thus, up to a diffeomorphism (possibly on the double cover of $S^{1} \times B^{3}$ ) the form $\omega_{X}$ is given by (1.2) to leading order in the distance to $\omega_{X}^{-1}(0)$. (Note that this double cover is required when the negative eigenspace of the matrix $\left(A_{i j}\right)$ in (1.14) defines the unoriented line bundle over $S^{1}$. As pointed out by Luttinger [12], an example for the latter case occurs when $\omega_{X}$ is the self-dual form on $\mathbb{R}^{4}=\mathbb{C}^{2}$ for the standard Euclidian metric whose expression in complex coordinates $\left(w_{1}, w_{2}\right)$ is

$$
\begin{aligned}
& i\left(1-\left|w_{1}\right|^{2}+\left|w_{2}\right|^{2}\right)\left(d w_{1} \wedge d \bar{w}_{1}+d w_{2} \wedge d \bar{w}_{2}\right) \\
& +i\left(R^{-1} w_{2}+\bar{w}_{1} \bar{w}_{2}\right) d w_{1} \wedge d w_{2} \\
& -i\left(R^{-1} \bar{w}_{2}+w_{1} w_{2}\right) d \bar{w}_{1} \wedge d \bar{w}_{2} .
\end{aligned}
$$

Here, take $R>>1$. The zero set of this form consists of the unit circle in the plane where $w_{2}=0$ and another circle where $\left|w_{1}\right|=R$. The negative eigenspace of the matrix $\left(A_{i j}\right)$ for the former component is unoriented.)

Moser's trick can be used (the details are written down in [10]) to make an additional coordinate change so that $\omega=\omega_{X}$ near where $\omega_{X}=0$ (perhaps on the double cover of $S^{1} \times B^{3}$ ). However, the latter change of coordinates may not be $\mathbb{C}^{\infty}$.

Alternately, $\omega_{X}$ can be homotoped through forms with fixed, non-degenerate vanishing set which are symplectic on the complement of this fixed vanishing set, and so that the end result is a closed 2-form which agrees with (1.2)'s form $\omega$ (up to a diffeomorphism on $S^{1} \times B^{3}$ or its double cover) near where $\omega_{X}=0$. Note that this homotopy can be made so as to have non-trivial support in any 
given neighborhood of fixed vanishing set. Furthermore, one can prove directly that the form at the end of the homotopy is self-dual with respect to a smooth metric, $g_{X}$, on $X$ which, on the given $S^{1} \times B^{3}$ or its double cover, is the standard metric near $S^{1} \times\{0\}$. (See also [10] for this last point.)

A form $\omega_{X}$ as just described has a compatible almost complex structure, $J_{X}$, whose restriction to the given $S^{1} \times B^{3}$ (or to its double cover) is equal to $J$ from (1.4) in the coordinates where the form is given by (1.2). Indeed, take the metric $g_{X}$ from the preceding paragraph and set $J_{X}=\sqrt{2} g_{X}^{-1} \omega_{X} /\left|\omega_{X}\right|$.

Given any $\omega_{X}$-compatible almost complex structure $J_{X}$ for $Y=X-\omega_{X}^{-1}(0)$, the notion of a finite energy, pseudo-holomorphic subvariety is defined as in Definitions 1.1 and 1.2 using $\omega=\omega_{X}$ and $J=J_{X}$.

By restriction to a neighborhood of $\omega_{X}^{-1}(0)$, Theorem 1.8 describes such finite energy, pseudo-holomorphic subvarieties in the case when there are coordinates $\left(t, x_{1}, x_{2}, x_{3}\right)$ for each component of a tubular neighborhood (or its double cover) of $\omega_{X}^{-1}(0)$ with the property that both $\omega_{X}=\omega$ from (1.2) and $J_{X}=J$ from (1.4). Theorem 1.8 says nothing directly in the case where $\omega_{X}$ and $J_{X}$ is not as strongly constrained near $\omega_{X}^{-1}(0)$.

The study of finite energy, pseudo-holomorphic subvarieties in the more general case will be deferred to a (planned) sequel to this article. However, note that [22] proves that pseudoholomorphic subvarieties exist in $X-Z$ when $X$ has a nonzero Seiberg-Witten invariant. Also [18] and [23] contain various speculative remarks about the significance of the pseudoholomorphic varieties studied here in the context of 4-manifold differential topology.

\section{1.d More about Example 1.5}

The purpose of this subsection is to prove various assertions in the discussion surrounding the solutions to (1.6) and the corresponding pseudo-holomorphic submanifolds. These assertions are restated as Lemmas 1.9 and 1.10. This subsection can be skipped at first reading.

Lemma 1.9 Let $c \in\left(0,2 / 3^{3 / 2}\right]$. Then, up to the action of translation on $\mathbb{R}$, there is a unique solution, $u$, to (1.6) which obeys $\frac{9}{4}\left(u^{\prime}\right)^{2}+u^{2}+c / u=1$ and is somewhere positive. Furthermore, $u$ is a periodic function of $t$ and there exist $c \neq 2 / 3^{3 / 2}$ for which the period is a rational multiple of $\pi$. Indeed, the assignment to $c$ of the period of the associated solution $u$ defines a non-constant, analytic function on $\left(0,2 / 3^{3 / 2}\right)$. 
Lemma 1.10 There is a constant $\zeta \geq 1$ with the following significance: Suppose that $c \in\left(0,2 / 3^{3 / 2}\right]$ is such that the corresponding solution to (1.6) has rational periods. Let $\Sigma_{ \pm}$denote the corresponding submanifold as described in (1.7). Let $r>0$ and let $B_{r} \subset S^{1} \times B^{3}$ denote the ball with center $\left(t_{0}, 0\right)$ and radius $r$. Then the integral of $\omega$ over $\Sigma_{ \pm} \cap B_{r}$ has the form $r^{3} \delta_{c}$ where $\delta_{c} \geq \zeta^{-1}$.

The remainder of this subsection is occupied with the proofs of these two lemmas.

Proof of Lemma 1.9 To find a solution to (1.6), consider first the same equation where $c=1$. Write the latter as the dynamical system:

- $u^{\prime}=p$

- $p^{\prime}=-\frac{4}{9} u+\frac{2}{9} u^{-2}$

Standard existence and uniqueness theorems for integrating vector fields on the plane imply that each point in the $(u, p)$ plane where $u>0$ sits on a unique trajectory of (1.16). Moreover, the function $\frac{4}{9} p^{2}+u^{2}+1 / u$ is constant on each trajectory. (Just differentiate and use (1.16).) This constant is necessarily positive on a trajectory where $u>0$. Then, the constancy of $\frac{4}{9} p^{2}+u^{2}+1 / u$ along trajectories implies that each trajectory is compact and thus diffeomorphic to a circle in the $u>0$ portion of the $(u, p)$ plane. In terms of (1.6), the preceding implies that the $\mathrm{c}=1$ version of (1.6) has a unique $u>0$ solution with given initial conditions $\left(u(0)=u_{0}>0, u^{\prime}(0)=p_{0}\right)$. Moreover, this solution is periodic.

Now, suppose that $(u, p)$ obey (1.16) and $\frac{4}{9} p^{2}+u^{2}+1 / u=e^{2}$ for some $e>0$. (By the way, note that $u^{2}+1 / u \geq 3 / 2^{2 / 3}$ and so $e \geq 3^{1 / 2} / 2^{1 / 3}$ with equality if and only if $u=1 / 2^{1 / 3}$.) Then $\underline{u} \equiv u / e$ obeys (1.6) with the normalization $\frac{4}{9} \underline{u}^{\prime 2}+\underline{u}^{2}+c / \underline{u}=1$ with $c=e^{-3}$. (The preceding lower bound on $e$ implies the upper bound $c \leq 2 / 3^{3 / 2}$.) Conversely, if $\underline{u}$ obeys (1.6) with the normalization, $\frac{4}{9} \underline{u}^{\prime 2}+\underline{u}^{2}+c / \underline{u}=1$ then $u=e \underline{u}$ obeys (1.16) for $c=1$ and with the normalization $\frac{4}{9} p^{2}+u^{2}+1 / u=c^{-2 / 3}$. With this last fact understood, the existence and uniqueness theorems for (1.16) directly imply the asserted existence and uniqueness for solutions to (1.6).

Now consider the assertion that there exists solutions with periods which are rational multiples of $\pi$. Since the period depends continuously on the parameter $c$, it is sufficient to prove that the period is not constant. The proof of this fact below will also establish the final assertion of the lemma, namely that the period defines a non-constant, analytic function on $\left(0,2 / 3^{3 / 2}\right)$. 
To begin, note that the period equals twice the time it takes for $u$ to travel from its minimum value to its maximum value, and the latter time is equal to

$$
\frac{3}{2} \int_{u_{\min }}^{u_{\max }}\left(1-x^{2}-c / x\right)^{-\frac{1}{2}} d x .
$$

Thus, to estimate the period, estimates for the integral in (1.17) are required. For this purpose, note that $u_{\min }<u_{\max }$ are the two positive roots of the polynomial $x^{3}-x+c$. (For $c \in\left(0,2 / 3^{3 / 2}\right]$, this polynomial has two positive roots and one negative root.) To find these roots, note that when $c=2 / 3^{3 / 2}$, the polynomial has a double root at $1 / 3^{1 / 2}$ and a single root at $-2 / 3^{1 / 2}$. In particular, the roots have the form $\lambda \pm \delta,-2 \lambda$, where $\lambda, \delta>0$ and $\delta<\lambda$. Moreover, when $c$ is close to $2 / 3^{3 / 2}$, then $\lambda$ is close to $1 / 3^{1 / 2}$ and $\delta$ is close to zero. With the preceding understood, one can rewrite (1.17) by changing variables to $y=(x-\lambda) / \delta$. The resulting integral is

$$
3^{\frac{1}{2}} / 2 \int_{[-1,1]}\left(1-y^{2}\right)^{-\frac{1}{2}}(1+\delta y / \lambda)^{\frac{1}{2}}(1+\delta y /(3 \lambda))^{-\frac{1}{2}} d y .
$$

Note that as $0<\delta / \lambda<1$, this integral is an analytic function of the ratio $\delta / \lambda$. This combination is an analytic function of the roots of the polynomial $x^{3}-x+c$, and thus an analytic function of $c$ except at values of $c$ where the roots are not distinct. The latter does not occur in the interval $\left(0,2 / 3^{3 / 2}\right)$.

Thus, it remains only to prove that (1.18) is not a constant function of c. To argue this point, consider this last integral as a function of $\delta / \lambda$. As such, it can be written as a power series $\delta / \lambda$ when $|\delta / \lambda|<<1$. In particular, to order $(\delta / \lambda)^{2}$, the integral in (1.18) is equal to

$$
3^{\frac{1}{2}} / 2 \int_{[-1,1]}\left(1-y^{2}\right)^{-\frac{1}{2}}\left[1-\delta^{2} y^{2} /\left(6 \lambda^{2}\right)\right] d y .
$$

This last integral can be evaluated using the trigonometric substitution $y=$ $\cos (\theta)$ and yields

$$
3^{\frac{1}{2}} / 2 \pi\left[1-\delta^{2} /\left(12 \lambda^{2}\right)\right] .
$$

Note that (1.20) already implies that the period is not a constant function of $c$ since $\delta^{2} / \lambda^{2}$ is not a constant function of $c$. (Note that $\delta / \lambda=0$ at $c=2 / 3^{3 / 2}$ but not otherwise.)

Although this last observation completes the proof of Lemma 1.9, a digression follows (for the sake of completeness) which uses (1.20) to estimate the period as a function of $\mathrm{c}$ which is accurate to order $\left(2 / 3^{3 / 2}-c\right)^{3 / 2}$. The digression starts with a determination of the constants $\delta$ and $\lambda$ in terms of $\alpha$. For this purpose, it is necessary to find a dependence of the roots of polynomial $x^{3}-x+2 / 3^{3 / 2}-\alpha$. 
For small $\alpha$, it turns out that these roots can be expanded as a power series $\alpha^{1 / 2}$. In particular, for small $\alpha$, the roots are

$$
1 / 3^{\frac{1}{2}} \pm 3^{-\frac{1}{4}} \alpha^{\frac{1}{2}}-\alpha / 6+\mathcal{O}\left(\alpha^{\frac{3}{2}}\right) \text { and }-2 / 3^{\frac{1}{2}}+\alpha / 3+\mathcal{O}\left(\alpha^{2}\right) .
$$

(This last assertion can be checked by substitution.) Note that this is an expansion in $\sqrt{\alpha}$ rather than $\alpha$ because the expansion is being done around the value $c=2 / 3^{3 / 2}$ where the polynomial $x^{3}-x+c$ has a double root. In any event, these last expressions identify $\lambda=1 / 3^{1 / 2}-\alpha / 6$ and $\delta=3^{-1 / 4} \alpha^{1 / 2}$. Then, with these last identifications and (1.20) understood, the period for small $\alpha$ is seen to be

$$
3^{\frac{1}{2}} \pi[1-\alpha /(4 \sqrt{3})]+\mathcal{O}\left(\alpha^{\frac{3}{2}}\right) .
$$

Proof of Lemma 1.10 As remarked previously, because $\omega$ is a homogeneous function of the coordinates $(t, x, y, z)$ and because $\Sigma_{ \pm}$is a cone, it follows that the integral of $\omega$ over $\Sigma_{ \pm} \cap B_{r}$ is finite and has the form $r^{3} \delta_{c}$ where $\delta_{c}$ is independent of $r$. Thus, it remains only to bound $\delta_{c}$ from below with a positive, $c$-independent constant. For this purpose, consider the case where $c>0$. (The $c<0$ case is treated almost identically.) Now write $\omega$ as in (1.10). Thus, the integral of $\omega$ over $\Sigma_{ \pm} \cap B_{1}$ is a sum of two integrals, the first being that of $d t \wedge d f$ and the second that of $d \phi \wedge d h$.

The integral of the latter is equal to $2 \pi b c$ as the following argument shows: First, the second and third points of (1.7) assert that $h=c s^{3}$ on $\Sigma_{+}$. Thus, $d h=c s^{2} d s$ on $\Sigma_{+}$. With this understood, Stoke's theorem writes the integral of $d \phi \wedge d h$ over $\Sigma_{+} \cap B_{1}$ as $c$ times the integral of $\phi^{\prime} d \tau$ over $[0,2 \pi b]$. (This last expression is the pull-back via the $\tau$ parametrization of the restriction of $d \phi$ to $\left.\Sigma_{+} \cap B_{1}\right)$. The latter integral is $2 \pi b$, a fact which follows from the fourth point in (1.7).

Given the preceding paragraph, a positive lower bound for $\delta_{c}$ follows from the following claim: There exists a constant $\xi \geq 1$ with the property that $\xi^{-1}$ bounds the integral of $d t \wedge d f$ over $\Sigma_{+} \cap B_{1}$ when $c<\xi^{-1}$.

To prove this claim, use (1.7) and Stoke's theorem to write the integral of $d t \wedge d f$ over $\Sigma_{+} \cap B_{1}$ as the integral over the interval $[0,2 \pi b]$ of the function $(3 / 4)\left(2 u^{2}-\right.$ $c / u) u^{\prime \prime}$. (This last expression is the pull-back via the t parametrization of the restriction of $-f d t$ to $\Sigma_{+} \cap B_{1}$.) Next, use (1.6) to write this last integral as

$$
\int_{[0,2 \pi b]} 3^{-1} u^{-1}\left(2 u^{2}-c / u\right)^{2} d \tau
$$

To proceed further, consider the maximum value for $u$ on $[0,2 \pi b]$. Since $u^{\prime}=0$ at this point, it follows from the normalization $\frac{9}{4}\left(u^{\prime}\right)^{2}+u^{2}+c / u=1$ that 
$u^{2}+c / u=1$ at maximum. Moreover, since $u^{\prime \prime} \leq 0$, one can conclude from (1.6) that $u^{2} \geq c / 2 u$ at maximum. Thus, $u^{2} \geq 1 / 3$ at its maximum. Now, let $\tau_{0}$ be a point at which $u$ achieves this maximum. Since the aforementioned normalization condition implies that $\left|u^{\prime}\right| \leq 2 / 3$, it follows that $u \geq 1 /(2 \sqrt{3})$ at all $t$ with $\left|\tau-\tau_{0}\right| \leq \sqrt{3 / 4}$. Since $u \leq 1$ (because of the normalization condition), these last points imply that the integral in (1.23) is no smaller than

$$
(144 \sqrt{3})^{-1}(1-12 \sqrt{3} c)^{2}
$$

when $c<\sqrt{3 / 12}$. Thus, when $c<\sqrt{3 / 24}$, the integral of $d t \wedge d f$ over $\Sigma_{+} \cap B_{1}$ is no smaller than $(576 \sqrt{3})^{-1}$.

\section{$2 \quad$ Finite energy and finite local energy}

The key step in analyzing the behavior near $Z$ of a finite energy, pseudoholomorphic subvariety $C$ is the local estimate given in Proposition 2.1, below, for the amount of "energy" which can accumulate in a small ball about any point in $Z$.

To start, fix a point, $t_{0}$, in $Z$ which can be taken to be the origin in $S^{1}=\mathbb{R} / \mathbb{Z}$. Let $\chi$ be a fixed "bump" function on $[0, \infty)$; that is, $\chi$ is a function which is non-increasing, equals 1 on $[0,1)$ and equals 0 on $[2, \infty)$. Given $s>0$, promote $\chi$ to a function $\chi_{s}$ on $X$ by the rule

$$
\chi_{s}=\chi(r / s)
$$

where $r=\left(t^{2}+\rho^{2}+z^{2}\right)^{1 / 2}$.

Now, for each $s>0$ introduce the "local" energy

$$
\sigma(s)=\int_{c} \chi_{s} \omega .
$$

This section proves the following estimate for $\sigma(s)$ :

Proposition 2.1 There exists $\zeta>1$ such that for any point $t_{0} \in Z$, the function $\sigma$ is differentiable on $\left(0, \zeta^{-1}\right)$. Furthermore, $\sigma$ obeys the inequalities:

(1) $\sigma(s) \leq z \cdot s^{3}$

(2) The area of $C \cap B(s)$ is bounded by $\zeta \cdot s^{2}$.

(3) $\frac{d}{d s} \sigma(s) \geq \frac{3}{s} \sigma(s)$ 
Here is an immediate corollary, a bound for the energy and area of the intersection of $C$ with a tubular neighborhood of $Z$ :

Proposition 2.2 Let $C$ be a finite energy, pseudo-holomorphic submanifold. Then there exists $\zeta$ with the following significance: Fix $s>0$ and let $C(s)$ denote the intersection of $C$ with the subset of $X$ where $\left(\rho^{2}+z^{2}\right)^{1 / 2}<s$. Then:

(a) $\int_{C(s)} \omega \leq \zeta \cdot s^{2}$

(b) The area of $C(s)$ is bounded by $\zeta \cdot s$.

The remainder of this section is occupied with the proof of Proposition 2.1.

Proof of Proposition 2.1 Consider first the issue of the differentiability of the function $\sigma$. In this regard, it proves useful to introduce a second bump function: Given $\varepsilon>0$, define the function $\eta_{\varepsilon}$ on $X$ by the rule $\eta_{\varepsilon}=(1-$ $\left.\chi\left(\left(\rho^{2}+z^{2}\right)^{1 / 2} / \varepsilon\right)\right)$. Note, in particular, that the support of $\eta_{\varepsilon}$ has closure which is disjoint from $Z$.

Next, note that for $s$ fixed, the function of $\varepsilon$ given by

$$
\sigma(s, \varepsilon)=\int_{c} \eta_{\varepsilon} \cdot \chi_{s} \cdot \omega
$$

is a decreasing function of $\varepsilon$ with

$$
\lim _{\varepsilon \rightarrow 0} \sigma(s, \varepsilon)=\sigma(s) .
$$

(Remember that the restriction of $\omega$ to $C$ is equal to $g$ times the induced area form on $C$ since $C$ is pseudo-holomorphic.) Note as well that the limit in question is uniform in $s$. Infact, given $\delta>0$, there exists $\varepsilon(\delta)$ such that for all $\varepsilon, \varepsilon^{\prime}<\varepsilon(\delta)$, and all $s$, one has

$$
\left|\sigma(s, \varepsilon)-\sigma\left(s, \varepsilon^{\prime}\right)\right|<\delta .
$$

Indeed, (2.6) stems from the following observations: First, because $C$ is pseudoholomorphic, the restriction of $\omega$ to TC is a positive multiple of the area form of the induced metric. In fact, this multiple is the function $g$ in (1.3). Thus, when $\varepsilon>\varepsilon^{\prime}$, then the difference in (2.6) is not greater than the integral of $\omega$ over the subset of points in $C$ where $\left(\rho^{2}+z^{2}\right)^{1 / 2} \leq 2 \cdot \varepsilon$. And, the latter can be made as small as desired by choosing $\varepsilon$ small since the integral over $C$ of $\omega$ is assumed finite.

By inspection the function $\sigma(\cdot, \varepsilon)$ is continuous for fixed $\varepsilon$, and so it follows from (2.6) that $\sigma$ is too. 
As for the derivative of $\sigma$, a similar argument finds $\sigma$ continuously differentiable with

$$
\sigma^{\prime}(s)=s^{-2} \cdot \int_{c} \chi_{s}^{\prime} \cdot r \cdot \omega .
$$

(Note that the right hand side in (2.7) is no greater than $\zeta \cdot s^{-1} \cdot \sigma(2 s)$ where $\zeta$ is independent of $s$.)

As for the estimates in (2.3), remark first that the first and second estimates follow by integration from the third and from the fact that $\omega$ on TC is $g$ times the area form. To consider the estimate in (2.3.3), introduce the 1-form

$$
\theta=3^{-1}(t \cdot d f-2 f \cdot d t-3 h \cdot d \phi) .
$$

Note that $d \theta=\omega$. Thus, integration finds

$$
\begin{aligned}
\sigma(s, \varepsilon) & =-\int_{c} \eta_{\varepsilon} \cdot d \chi_{s} \wedge \theta-\int_{c} \chi_{s} \cdot d \eta_{\varepsilon} \wedge \theta \\
& \leq s^{-1} \int_{c} \chi_{s}^{\prime} \cdot \kappa \cdot|\theta| \cdot g^{-1} \cdot \omega+\int_{c}\left|d \eta_{\varepsilon}\right| \cdot|\theta| \cdot g^{-1} \cdot \omega .
\end{aligned}
$$

Here, $\kappa$ is the norm of pull-back to $C$ of the 1 -form $|d r|$. (The right most inequality in (2.9) exploits the fact that $\omega$ on TC is equal to $g$ times the area form of the induced metric.)

To make use of (2.6), remark that a calculation finds,

$$
|\theta| \leq 3^{-1} \cdot\left(t^{2} \cdot|d f|^{2}+4 \cdot|f|^{2} \cdot|d t|^{2}+9 \cdot|h|^{2} \cdot|d \phi|^{2}\right)^{12} \leq 3^{-1} \cdot r \cdot g .
$$

(The second inequality follows from the identity

$$
t^{2} \cdot|d f|^{2}+4 \cdot|f|^{2} \cdot|d t|^{2}+9 \cdot|h|^{2} \cdot|d \phi|^{2}=\left(t^{2}+\rho^{2}+z^{2}\right) \cdot g^{2}
$$

which holds on $S^{1} \times \mathbb{R}^{3}$.) Thus, according to (2.9) and (2.10),

$$
\sigma(s, \varepsilon) \leq 3^{-1} \cdot s^{-1} \cdot \int_{c} \chi_{s} \cdot \kappa \cdot r \cdot \omega+\zeta \cdot \int_{c} \eta_{\varepsilon / 4} \cdot\left(1-\eta_{4 \varepsilon}\right) \cdot \omega .
$$

As $\varepsilon \rightarrow 0$, the second term on the right side in (2.12) vanishes, while the first term on the right side is less than $s \cdot \sigma^{\prime}(s) / 3$ since $\kappa \leq 1$ always. (Compare with (2.7).)

\section{Limits}

Fix a point $t_{0} \in Z$ as in the previous section, and again call this point 0 . For each positive $s$, introduce $B(s)=\left\{(t, x, y, z): r=\left(t^{2}+x^{2}+y^{2}+z^{2}\right)^{1 / 2}<s\right\}$. Then, for $R \geq 2$ and for any $s<R / 2$, define $C_{s} \subset B(R)$ by the rule

$$
C_{s}=\{(t, x, y, z) \in B(R): s \cdot(t, x, y, z) \in C \cap B(R \cdot s)\} .
$$


Of course, $C_{s}$ is obtained from $C \cap B(R \cdot s)$ by dilating all of the coordinates. Since $\omega$ is homogeneous under dilation, and the metric as well, it follows immediately that $C_{s}$ is a $J$-pseudo-holomorphic subvariety of $B(R)$. Furthermore, it follows from Proposition 2.1 that the integral over this subvariety of $\omega$ is bounded independently of $s$. Also, the area of $C_{s}$ is bounded independently of $s$.

With the preceding understood, one can consider the limiting behavior as $s \rightarrow 0$ of the family $\left\{C_{s}\right\}$. The next definition describes a useful notion of convergence. However, a preliminary digression is required before this definition. The purpose of the digression is to introduce the notion of an irreducible component of a pseudo-holomorphic subvariety. For this purpose, suppose that $C$ is a finite energy, pseudo-holomorphic subvariety in a symplect 4-manifold $Y$ with compatible almost complex structure. By definition, there is a countable set $\Lambda \subset C$ whose complement is a submanifold of $Y$. Take any such set $\Lambda$. An irreducible component of $C$ is the closure of a component of $C-\Lambda$. (The set of irreducible components is insensitive to the choice of $\Lambda$ provided that $\Lambda$ is countable and $C-\Lambda$ is a submanifold.)

Note that each irreducible component of a finite energy, pseudo-holomorphic subvariety is also a finite energy, pseudo-holomorphic subvariety. (The sum of the energies of the irreducible components of $C$ equals that of $C$.)

End the digression. Here is the promised convergence definition:

Definition 3.1 Let $Y$ be a (possibly non-compact) 4-manifold with symplectic form $\omega$ and with a compatible almost complex structure $J$. Let $\Omega$ be a countable, decreasing sequence of positive numbers with limit zero and let $\left\{C_{s}\right\}_{s \in \Omega} \subset Y$ be a corresponding sequence of finite energy, pseudo-holomorphic subvarieties. Let $C \subset Y$ be a finite energy, pseudo-holomorphic subvariety as well. Say that $\left\{C_{s}\right\}$ converges geometrically to $C$ when the following two requirements are met:

- Treat each $C_{s}$ and also each irreducible component of $C$ as a 2-dimensional, rectifiable current which assigns to a smooth 2-form with compact support on $Y$ the integral of the form over the set in question. Let $\mathcal{C}$ denote the set of irreducible components of $C$. Then, require that the sequence of currents $\left\{C_{s}\right\}$ converges weakly as a current to the current $\Sigma_{C^{\prime} \in \mathcal{C}} m_{C^{\prime}} C^{\prime}$, where $m_{C}$ is, for each $C \in \mathcal{C}$, a positive integer.

- For each compact subset $K \subset Y$, introduce the number, $d_{K}(s)$ which is the supremum over pairs $(x, y) \in\left(C_{s} \cap K\right) \times\left(C_{0} \cap K\right)$ of the sum of the distances 
from $x$ to $C_{0}$ and $y$ to $C_{s}$. Then, for each such $K$, require that the limit as $s \rightarrow 0$ of $d_{K}(s)$ exists and is zero.

With the preceding definition understood, here is the main result of this section:

Proposition 3.2 Let $\left\{C_{s}\right\}_{s>0}$ be defined as in (3.1). Let $\left\{s_{i}\right\}$ be a countable, decreasing sequence of $s$ values with limit zero. This sequence has a subsequence for which the corresponding set $\left\{C_{s}\right\}$ geometrically converges in each $Y=B(2)-Z$ to a finite energy, pseudo-holomorphic submanifold $C_{0} \subset B(2)-Z$. Furthermore, each connected component of $C_{0}$ in $B(1)-Z$ is identical to the intersection of $B(1)-Z$ with one of the submanifolds from Example 1.3, 1.4 or 1.5.

The proof of this proposition exploits the following local version of Gromov's compactness theorem [5] (see also [16], [17], [24], [14]).

Proposition 3.3 Let $Y$ be a smooth 4-manifold with symplectic form $\omega$ and compatible almost complex structure $J$. Let $\Omega$ be a countable, decreasing set of real numbers with limit zero and let $\left\{C_{s}\right\}_{s \in \Omega} \subset Y$ be a corresponding set of finite energy, pseudo-holomorphic subvarieties. Suppose that there exists $E>0$ such that $\int_{C} \omega<E$ for all $C \subset\left\{C_{s}\right\}$. Then there is a finite energy, pseudo-holomorphic subvariety $C_{0} \subset Y$ and an infinite subsequence of $\Omega$ for which the corresponding subset $\left\{C_{s}\right\}$ converges geometrically to $C_{0}$.

This last proposition is proved at the end of this section. Accept it momentarily for the proof of Proposition 3.2.

Proof of Proposition 3.2 As remarked above, the sequence $\left\{C_{s}\right\}$ has uniformly bounded energy, so Proposition 3.3 provides a subsequence which converges geometrically to the limit $C_{0}$. As $C_{0}$ has finite energy, the results of Section 2 apply to the subvariety $C_{0}$. For any $a \in(0,1)$, consider the value of the function $\sigma()$ on $C_{0}$ at the point $a$. (Take the point $t_{0}=0 \in Z$ to define this function.) By the convergence criteria, $\sigma(a)$ for $C_{0}$ is the limit as $s \rightarrow 0$ of the values of $m^{-1} \sigma(a)$ for $C_{s}$ where $m$ is a positive integer. And, by rescaling, this is equal to

$$
m^{-1} \cdot(R s)^{-3} \cdot \sigma(R \cdot s \cdot a)
$$

as defined for the curve $C$.

On the other hand, the function $s^{-3} \sigma(s)$ on $C$ is a monotonically increasing function of $s$, which means, since it is bounded, that there is a unique limit, 
$\sigma_{0}$, as $s \rightarrow 0$. Thus, as $s$ tends to zero, (3.2) converges to $a^{3} \cdot m^{-1} \cdot \sigma_{0}$. As a result, one can conclude that the value of $\sigma(\cdot)$ on $C_{0}$ at the point $a$ is equal to $a^{3} \cdot m^{-1} \cdot \sigma_{0}$, with $\sigma_{0}$ being independent of $a$.

Meanwhile, according to Proposition 2.1, the derivative with respect to a of $a^{-3}$. $\sigma(a)$ is non-negative. In the case at hand, this derivative is zero. According to (2.7)-(2.12), this derivative is zero only if a number of inequalities are equalities. In particular, the function $\kappa$ in (2.12) must equal 1 everywhere on $C_{0}$ which can only happen when the vector field

$$
s \partial_{s}=t \partial_{t}+\rho \partial \rho+z \partial_{z}
$$

is everywhere tangent to $C_{0}$. Here, $s^{2}=t^{2}+\rho^{2}+z^{2}$. Then, $J \cdot\left(s \partial_{s}\right)$ must also be tangent to $C_{0}$. It then follows that $C_{0}$ is the cone on a union of integral curves of $J \cdot\left(s \partial_{s}\right)$. Furthermore, as no two distinct integral curves intersect, it follows that each component of $C_{0}$ is submanifold, and that no two distinct components intersect.

The analysis of the integral curves of $J \cdot\left(s \partial_{s}\right)$ is facilitated by writing the latter vector field as

$$
J \cdot\left(s \partial_{s}\right)=-g t \partial_{f}+2 g^{-1} f \partial_{t}+3\left(g \rho^{2}\right)^{-1} h \partial_{\phi}
$$

(This equation follows from (1.10) and (1.11) and the identity $s \partial_{s}=t \partial_{t}+$ $\left.2 f \partial_{f}+3 h \partial_{h}.\right)$ Now, remark that $J \cdot\left(s \partial_{s}\right)$ annihilates not only the function $s$, but also the function $h$. Thus, $h=$ constant on an integral curve of $J \cdot\left(s \partial_{s}\right)$. If $h=0$, then the resulting component of $C_{0}$ is one of the cases from Example 1.3 and Example 1.4. At most finitely many components of $C_{0}$ are described in this way because a calculation shows that each component contributes at least $2 r^{3} / 3$ to the integral of $\omega$ over $C_{0} \cap B_{r}$. Thus, Proposition 3.2 follows with a demonstration that each $h \neq 0$ case is described in Example 1.5, and that there are at most finitely many of these.

To begin the study of the $h \neq 0$ case, assume, for the sake of argument, $h>0$ on an integral curve $\gamma$ of (3.4). (The case $h<0$ is argued analogously.) Also, normalize the curve so that $s \equiv 1$ on $\gamma$.

The first observation is that the condition $h=$ constant $>0$ implies that the $\mathbb{R} /(2 \pi \mathbb{Z})$ valued function $\phi$ is increasing along $\gamma$. Thus, $\phi$ restricts to $\gamma$ as a local coordinate and the corresponding component of $C_{0}$ can be parametrized, at least locally, by setting $t=s \underline{t}(\phi), f=s^{2} \underline{f}(\phi)$ and $h=c s^{3}$, where $c$ is a positive constant and where $\underline{t}$ and $\underline{f}$ are functions of $\phi$ as indicated. 
The condition that $\gamma$ is an integral curve of $J \cdot\left(s \partial_{s}\right)$ implies that the functions $\underline{t}$ and $\underline{f}$ are constrained to obey the equations:

$$
\begin{array}{r}
3 c \underline{t}_{\phi}=2 \underline{\rho}^{2} \underline{f} \\
3 c \underline{f}_{\phi}=-\underline{g}^{2} \underline{\rho}^{2} \underline{t}
\end{array}
$$

Here, $\underline{g}$ and $\underline{\rho}$ are the restrictions of the the functions $g$ and $\rho$ to $\gamma$. To analyze (3.5), one can exploit the fact that both $\underline{t}$ and $\underline{f}$ along $\gamma$ can be written implicitly as functions of the restriction, $\underline{z}$, of $z$ to $\gamma$. (This is because $s$ and $h$ are constant on $\gamma$ ). Indeed, $\underline{\rho}^{2}=c / \underline{z}$ from the definition of $h$ and therefore $\mu=2^{-1} c / \underline{z}-\underline{z}^{2}$ while $\nu= \pm\left(1-c / \underline{z}-\underline{z}^{2}\right)^{1 / 2}$. Moreover, $\underline{g}^{2}=c / \underline{z}+4 \underline{z}^{2}$, so each line in (3.5) gives an equation for $\underline{z}_{\phi}$ in terms of $\underline{z}$. These two equations are identical and imply that $\underline{z}_{\phi}=2 \underline{t} / 3$. Differentiate the latter equation once to obtain a second order equation for the function $\underline{z}$. This second order equation is (1.6) with the substitution of $u$ for $\underline{z}$ and $t$ for $\phi$. Then, the description of the corresponding component of $C_{0}$ by (1.7) follows directly from the preceding remarks. Thus, the cases where $h \neq 0$ are described by Example 1.5 as required.

To complete the proof of Proposition 3.2, remark that the number of such $h \neq 0$ cases is finite as there is a $c$-independent lower bound for the integral of $\omega$ over the intersection with $B_{1}$ of any case from Example 1.5. (See Lemma 1.10.)

Proof of Proposition 3.3 The first step is to obtain the limit $C_{0}$ as a subset of Y. The following monotonicity lemma plays a key role in this step. (A similar lemma is proved in [17].)

Lemma 3.4 Let $Y$ be a smooth 4-manifold with symplectic form $\omega$ and almost complex structure $J$. Let $E$ be a positive number and let $K \subset Y$ be a compact set. Then, there exists a constant $c=c(E, K) \geq 1$ with the following significance: Let $C \subset Y$ be a finite energy, pseudo-holomorphic subvariety with $\int_{C} \omega \leq E$. Suppose that $x \in K \cap C$. Use $J$ and $\omega$ to define a Riemannian metric on $Y$, and for $r>0$, let $B(x, r) \subset Y$ denote the ball of radius $r$ with center $x$. Then,

$$
c^{-1} r^{2} \leq \int_{B(r, x) \cap C} \omega \leq c r^{2}
$$

if $r \leq c^{-1}$.

Proof of Lemma 3.4 Since $K$ is compact, there exists $r_{K}$ so that for each $x \in K$, the ball $B\left(r_{K}, x\right)$ has compact closure in $Y$, and so that in this coordinate system, $\omega=\omega_{0}+\omega_{1}$, where $\omega_{0}=d x_{1} \wedge d x_{2}+d x_{3} \wedge d x_{4}$ and where $\left|\omega_{1}\right| \leq|x|$. With the preceding understood, consider $f(r) \equiv \int_{B(x, r) \cap C} \omega$ and let 
$f_{0}(r)=\int_{B(x, r) \cap C} \omega_{0}$. Note that both functions are continuous and piecewise differentiable on the interval $\left[0, r_{K} / 2\right]$. Furthermore, as $f(r)$ is the area of the intersection of $C$ with $B(x, r)$, one has

$$
\left|f(r)-f_{0}(r)\right| \leq r \cdot f(r),
$$

as long as $r \leq r_{K} / 2$.

Meanwhile, $\omega_{0}=d \theta$ for $\theta=2^{-1}\left(x_{1} d x_{2}-x_{2} d x_{1}+x_{3} d x_{4}-x_{4} d x_{3}\right)$, and so

$$
f_{0}(r)=\int_{\partial B(x, r) \cap C} \theta \leq \int_{\partial B(x, r) \cap C}|\theta|=2^{-1} r \int_{\partial B(x, r) \cap C} 1=2^{-1} r f^{\prime}(r) .
$$

(In deriving the latter, note that the Euclidean ball of radius $r$ agrees with the Gaussian ball of radius $r$.) Thus, the function $f(r)$ obeys the differential inequality

$$
f(r) \leq(1+r) \cdot 2^{-1} r f^{\prime}(r) .
$$

The latter can be integrated to find that when $r \leq r^{\prime} \leq r_{K} / 2$. Then

$$
r^{\prime-2} f\left(r^{\prime}\right) \geq 2^{-1} r^{-2} f(r)
$$

when $r_{K} \leq 1$. Since $f\left(r^{\prime}\right) \leq E$, this last inequality implies first that $f(r) \leq$ $r^{2}\left(8 E r_{K}^{-2}\right)$ for any $r \leq r_{K} / 2$. This gives the right hand inequality in (3.6). The left-hand bound follows by taking $r$ very small on the right hand side of (3.10). In this case, standard regularity results about the local behavior of pseudo-holomorphic maps (as in [17] and [24]) imply that for $r>0$ but very small, $f(r) \geq \zeta^{-1} r^{2}$, where $\zeta$ is a fixed constant which is independent of all relevant variables. (The point here is that a pseudo-holomorphic subvariety looks locally like a complex subvariety in $\mathbb{C}^{2}$, and such a lower bound for $f(r)$ can be readily found for the latter.)

With Lemma 3.4 understood, the construction of $C_{0}$ proceeds with the following lemma:

Lemma 3.5 Let $Y$ be a smooth 4-manifold with symplectic form $\omega$ and almost complex structure $J$. Let $K \subset Y$ be a compact set and let $E$ be a positive number. Then, there exists a constant $c=c(E, K) \geq 1$ with the following significance: Let $C \subset Y$ be a finite energy, pseudo-holomorphic subvariety with $\int_{C} \omega \leq E$. Then given $r>0$ but less than $c^{-1}$, there is a set of no more that $\mathrm{cr}^{-2}$ balls of radius $r$ with center on $C \cap K$ which covers $C \cap K$. Furthermore, this set can be taken so that the concentric balls with radius $r / 4$ are pairwise disjoint. 
Proof of Lemma 3.5 First, choose $r_{K}$ so that for each $x \in K$, the ball $B\left(r_{k}, x\right)$ has compact closure in $Y$. Then, for $r$ positive, but less than $r_{K}$, let $\Xi$ be a maximal set of balls of radius $r / 4$ with centers on $K$ which are pairwise disjoint. Let $c$ be the constant which occurs in Lemma 3.4. Then, there can be no more that $c E r^{-2}$ such balls because, according to Lemma 3.4, each ball contributes at least $c^{-1} r^{2}$ to the energy. Take the cover to be the set of balls of radius $r$ which are concentric to the balls in $\Xi$. (If this set did not cover $C \cap K$, then $\Xi$ would not be maximal.)

With Lemma 3.5 understood, obtain $C_{0}$ by the following limiting argument: Take an exhaustion of $Y$ by a countable, nested set of compact sets $\left\{Y_{p}\right\}_{p=1,2, \ldots}$. Here, $Y_{p} \subset Y_{p+1}$ and $\cup_{p} Y_{p}=Y$. For each $p$ and integer $N$ sufficiently large, and for each $s \in \Omega$, choose a set $\Xi_{p, N, s}$ of no more than $c_{p} 4^{2} 16^{2 N}$ balls of radius $4^{-1} 16^{-N}$ which cover $C_{s} \cap Y_{p}$ and which have centers on $C_{s} \cap Y_{p}$. (Here, take $N$ large enough so that Lemmas 3.4 and 3.5 can be invoked for the case where $K=Y_{p}$ and $r=4^{-1} 16^{-N}$.) Add extra balls if required so that the number of elements in $\Xi_{p, N, s}$ is some $s$ independent integer $m_{p, N} \leq c_{p} 4^{2} 16^{2 N}$. Label the centers of these balls to give a labled set $\underline{x}_{p, n, s}$ of $m_{p, N}$ points in $Y_{p}$ which one can think of also a single point in the $m_{p, N}$ fold product of $Y_{p}$. For each fixed $p$ and $N$, the sequence $\left\{\underline{x}_{p, N, s}\right\}_{s \in \Omega} \subset \times_{m_{p, N}} Y_{p}$ has a convergent subsequence. By taking a diagonal subsequence, one obtains a subsequence $\Omega^{\prime} \subset \Omega$ for which each sequence $\left\{\underline{x}_{p, N, s}\right\}_{s \in \Omega^{\prime}}$ converges for each $p$ and $N$. Let $\underline{x}_{p, N, 0}$ denote the limit point, but thought of as a labeled set of $m_{p, N}$ points in $Y$. Let $U_{p, N, 0} \subset Y$ denote the set of points with distance $16^{-N}$ or less from some point in $\underline{x}_{p, N, 0}$. The claim now is that given $p$, there exists exists $N(p)$ such that

$$
U_{p^{\prime}, N+1,0} \cap Y_{p-1} \subset U_{p, N, 0}
$$

for all $p^{\prime}$ and for all $N \geq N(p)$. Given this claim, take

$$
C_{0} \cap Y_{p-1} \equiv \bigcap_{N \geq N(p)}\left(U_{p, N, 0} \cap Y_{p-1}\right) .
$$

Note that (3.11) insures that (3.12) is consistent with respect to the inclusion $Y_{p} \subset Y_{p+1}$.

To see (3.11), suppose to the contrary that $U_{p^{\prime}, N+1,0} \cap Y_{p-1}$ had a point which was not in $U_{p, N, 0}$. Then, for all small $s \in \Omega^{\prime}$, there would be a point $x \in Y_{p-1}$ with distance $8^{-1} 16^{-N}$ or less from some point in $\underline{x}_{p^{\prime}, N+1, s}$ and which had distance $2^{-1} 16^{-N}$ or more from each point in $\underline{x}_{p, N, s}$. However, this is not possible if $N$ is large for the following reason: Let $d_{p}$ denote the distance between $Y-Y_{p}$ and $Y_{p-1}$. Let $x^{\prime} \in \underline{x}_{p^{\prime}, N+1, s}$ denote the point which is closest to $x$. Then $x^{\prime} \in C_{s}$. Furthermore, as $x \in Y_{p-1}$, then $x^{\prime} \in C_{s} \cap Y_{p}$ if $8^{-1} 16^{-N}<d_{p}$. 
Of course, if $N$ is large, this will be the case. On the other hand, if $x^{\prime} \in C_{s} \cap Y_{p}$, then $x^{\prime}$ has distance $4^{-1} 16^{-N}$ from each point in $\underline{x}_{p, N, s}$. Thus, the triangle inequality implies that

$$
\operatorname{dist}\left(x, \underline{x}_{p, N, s}\right) \leq 4^{-1} 16^{-N}+8^{-1} 16^{-N}<2^{-1} 16^{-N} .
$$

With $C_{0}$ defined by (3.12), the claim next is that:

- $C_{0}$ is closed.

- $C_{0} \cap Y_{p}$ has finite two dimensional Hausdorff measure.

- For each $p$,

$$
\lim _{s \in \Omega, s \rightarrow 0}\left(\sup _{y \in C_{s} \cap Y_{p}} \operatorname{dist}\left(y, C_{0}\right)+\sup _{y \in C_{0} \cap Y_{p}} \operatorname{dist}\left(y, C_{s}\right)\right)=0 .
$$

Indeed, $C_{0}$ is closed as it is the intersection of closed sets. The fact that $C_{0} \cap Y_{p}$ has finite two dimensional Hausdorff measure follows from the construction and the definition of Hausdorff measure. The second point in (3.14) follows from the left most inequality in (3.6).

As remarked previously, each $C_{s}$ can be thought of as a current, which is to say a linear functional on the space of smooth, compactly supported 2-forms on $Y$. As a current each $C_{s}$ has norm bounded by $E$. Since the unit ball in the space of currents is compact in the weak topology, it follows that there is a subsequence $\Omega^{\prime \prime} \subset \Omega^{\prime}$ for which the corresponding sequence of currents $\left\{C_{s}\right\}_{s \in \Omega^{\prime \prime}}$ converges to a current with norm no greater than $E$. The latter has support in $C_{0}$ in the sense that it annihilates any 2-form whose support avoids $C_{0}$. Use $\underline{C}_{0}^{\prime}$ to denote the limit current.

The final step in the proof of Proposition 3.3 argues the following two points:

- There is a complex curve $C_{0}^{\prime}$ with a proper, pseudo-holomorphic map $f^{\prime}$ into $Y$ which embeds each component off of a finite set of points and whose image is $C_{0}$.

- The current $\underline{C}_{0}^{\prime}$ is equal to the composition of pull-back by $f$ and then integration over the complex curve.

Proposition 3.3 now follows directly from (3.15). (The curve $C_{0}$ in Definition 1.1 is obtained by identifying components of $C_{0}^{\prime}$ with the same image.)

The assertions in (3.15) will be proved by invoking the analysis in Section 6 of [20]. In particular, the first point of (3.15) follows from Proposition 6.1 in [20] with a demonstration that $C_{0}$ has a positive cohomology assignment as defined in Section 6a of [20] and reproduced in the following paragraph. However, with regard to the application of Proposition 6.1 in [20], the reader should take 
note that the proof offered in [20] has errors which occur in Section 6e of the article. A corrected proof is provided in the revised version of this same article which appears in [21]. Also, remark that the compactness requirement for the ambient manifold in the statement of Proposition 6.1 can be dropped in this case without compromising the conclusions except to allow the complex curve to be non-compact as long the associated map $f^{\prime}$ is proper.

The proof that $C_{0}$ has a positive cohomology assignment proceeds as follows: The point is to assign an integer to each member of a certain subset of smooth maps from the standard, open unit disk $D \subset \mathbb{C}$ into $Y$. The allowed maps are called admissible for $C_{0}$, and a map $\sigma$ is admissible when $\sigma$ extends as a continuous map from the closure, $\underline{D}$, of $D$ into $Y$ which sends $\partial \underline{D}$ into $Y-C_{0}$. The assignment, $I(\sigma)$, of an integer to $\sigma$ must satisfy the following constraints:

- $I(\cdot)$ must be a homotopy invariant for a homotopy $h:[0,1] \times D \rightarrow$ $Y$ which extends as a continuous map from $[0,1] \times \underline{D}$ into $Y$ sending $[0,1] \times \partial \underline{D}$ into $Y-C_{0}$.

- If $\sigma$ is admissable and if $\theta: D \rightarrow D$ is a proper, degree $k$ map, then $I(\sigma \theta)=k I(\sigma)$.

- If $\sigma$ is admissable and if $\sigma^{-1}\left(C_{0}\right)$ is contained in a collection of disjoint disk $\left\{D_{\nu}\right\} \subset D$, and if each $D_{\nu}=\theta_{\nu}(D)$ where each $\theta_{\nu}$ is an orientation preserving embedding, then $I(\sigma)=\sum_{\nu} I\left(\sigma \cdot \theta_{\nu}\right)$.

- If $\sigma$ is admissable, image $(\sigma)$ intersects $C_{0}$ and is a pseudo-holomorphic embedding, then $I(\sigma)>0$.

To obtain such an assignment for $C_{0}$, remark first that each $C_{s}$ defines a positive cohomology assignment if the assignment $I(\sigma)$ for $C_{s}$ is defined to be the intersection number of $C_{s}$ with any sufficiently small perturbation of $\sigma$ which makes the latter an immersion near $C_{s}$. (The last point in (3.15) follows from the fact that pseudo-holomorphic submanifolds have strictly positive local intersection numbers. See [13].) This cohomology assignment for $C_{s}$ will be denoted below by $I_{s}(\cdot)$ to avoid confusion.

Now, with $\sigma$ fixed and admissable for $C_{0}$, then $\sigma$ will also be admissable for $C_{s}$ if $\sigma$ is sufficiently large. This follows from the last point of (3.14). Furthermore, as $\left\{C_{s}\right\}_{s \in \Omega^{\prime \prime}}$ converges to $C_{0}$ as a current when $s \rightarrow 0$, the values of $I_{s}(\sigma)$ for $s \in \Omega^{\prime \prime}$ become independent of $s$ as $s \rightarrow 0$. (The reason for this is as follows: One can first perturb $\sigma$ so that the latter is immersed near $C_{0}$. This will not affect the value of $I_{s}(\sigma)$ for small s by the first point in (3.16). Then, the value of $I_{s}(\sigma)$ can be computed by integrating a smooth form over $C_{s}$ which has support in some small tubular neighborhood of the image of $\sigma$.) 
The fact that $I_{s}(\sigma)$ becomes $\mathrm{s}$ independent for small $s$ in $\Omega^{\prime \prime}$ allows for the following definition of $I(\sigma)$ for $C_{0}: I(\sigma)=\lim _{s \in \Omega^{\prime \prime}, s \rightarrow 0} I_{s}(\sigma)$. The fact that $I(\cdot)$ obeys the points in (3.16) follows from the fact that these same points are obeyed by each $I_{s}$. In this regard, the only subtle point in (3.16) is the final one. And, the final claim in (3.16) follows directly from Lemma 3.6, below. Indeed, Lemma 3.6 implies that when $s$ is large, then $C_{s}$ intersects the image of a perturbation, $\sigma^{\prime}$, of $\sigma$ which is homotopic to $\sigma$ by a homotopy which is $C_{s}$ admissible, and which is pseudo-holomorphic near $\sigma^{\prime-1}\left(C_{s}\right)$. This makes $I_{s}\left(\sigma^{\prime}\right)>0$ and hence $I_{s}(\sigma)>0$ too. (Of course, the conclusion that $I_{s}(\sigma)$ is positive follows with apriori knowledge that $C_{s}$ intersects $\sigma(D)$ for large $s$.)

Lemma 3.6 Let $\sigma: D \rightarrow Y$ be a pseudo-holomorphic map and let $\varepsilon>0$ be given and let $D^{\prime} \subset D$ be the concentric disk of radius $1-\varepsilon$. Then, there is a neighborhood $U \subset Y$ of $\sigma\left(D^{\prime}\right)$ and for each point $y \in U$, there is a smooth map $\sigma_{y}: D \rightarrow Y$ with the following properties:

- $\operatorname{dist}\left(\sigma_{y}(z), \sigma(z)\right)<\varepsilon \quad$ for all $z \in D$, and $\sigma_{y}=\sigma$ near $\partial \underline{D}$,

- $\left.\sigma_{y}\right|_{D^{\prime}}$ is pseudo-holomorphic,

- $y \in \sigma_{y}\left(D^{\prime}\right)$.

The proof of Lemma 3.6 is deferred for a moment to complete the discussion of (3.15). In particular, remark that with a positive cohomology assignment for $C_{0}$ given, Proposition 6.1 in [20] (with the corrected proof in [21]) proves the first point in (3.15). The argument for the second point in (3.15) is straightforward, but will be omitted save for the following outline: First, as noted, a pseudo-holomorphic subvariety has a canonical, positive cohomology assignment which is defined by taking the intersection number with maps from the disk which avoid the singular points and have transversal intersections with the remainder. Second, the current defined by a pseudo-holomorphic subvariety via integration can be computed from the values of the corresponding, canonical positive cohomology assignment. Third, according to Proposition 6.1 in [20], the cohomology assignment given by intersection with an irreducible component of $C_{0}$ as a pseudo-holomorphic subvariety is some positive multiple of that given above for that component as the $s \rightarrow 0$ limit of $\left\{I_{s}(\cdot)\right\}_{s \in \Omega^{\prime \prime}}$. Fourth, the limits of $I_{s}$ were defined using the fact that the set $\Omega^{\prime \prime}$ was chosen so that the currents $\left\{C_{s}\right\}_{s \in \Omega^{\prime \prime}}$ converge as $s$ tends to zero.

With the preceding understood, the proof of Proposition 3.4 is completed with the proof of Lemma 3.6. 
Proof of Lemma 3.6 As might be expected, the argument is perturbative in nature. To start, introduce the bundle $\sigma^{*} T X \rightarrow D$. The latter admits a smooth map, $\phi$, into $X$ which is obtained by composing the tautological map to $T X$ with the Riemannian metric's exponential map. Thus, a section $\eta$ of $\sigma^{*} T X$ defines, via composition with $\phi$, a perturbation of $\sigma$, with the zero section giving $\sigma$ itself. Note that this composition sends any neighborhood of the zero section onto a neighborhood of $\sigma(D)$.

The condition that the resulting map, $4 \phi \cdot \eta$, is pseudo-holomorphic defines an equation for $\eta$ which is elliptic, at least for small $\eta$. This equation has the form

$$
\mathcal{D} \eta+\mathcal{R}(\eta, \nabla \eta)=0
$$

where $\mathcal{D}$ is an elliptic, first order operator, and where $\mathcal{R}$ is the remainder, and is small in the sense that $|\mathcal{R}(\lambda \eta, \lambda \nabla \eta)| \leq \zeta_{\eta}|\lambda|^{2}$ for $\lambda \in \mathbb{C}$. (See, eg Chapters 1 and 2 of [14].) Regarding $\mathcal{D}$, note that $J$ endows $\sigma^{*} T X$ with a complex structure, and with this understood, $\mathcal{D}$ is a zero'th order, $\mathbb{R}$-linear (not $\mathbb{C}$ linear) perturbation of the standard d-bar operator.

Given the preceding, the Lemma 3.6 follows from

Lemma 3.7 There exist constants $\zeta>1$ and $\delta>0$ such that for each point $z \in D^{\prime}$ and $\left.\eta_{z} \in \sigma^{*} T X\right|_{z}$ with $\left|\eta_{z}\right|<\delta$, there exists a solution $\eta$ to (3.17) over the concentric disk with radius $1-\varepsilon / 2$ which obeys $\eta(z)=\eta_{z}$ and $|\eta| \leq \zeta\left|\eta_{z}\right|$.

Proof of Lemma 3.7 There are three steps to the proof.

Step 1 Introduce the disks $D_{-1}, D_{0}$ and $D_{1}$ which are concentric to $D$ and have respective radii $1-\varepsilon / 16,1-\varepsilon / 8$ and $1-\varepsilon / 4$. Let $\kappa: D \rightarrow[0,1]$ be a smooth function with compact support on $D_{1}$ which equals 1 on the radius $1-\varepsilon / 2$ disk. Given $\kappa$, extend (3.17) to an equation over $D_{0}$ :

$$
\mathcal{D} \eta+\kappa \mathcal{R}(\eta, \nabla \eta)=0
$$

The point is that the latter equation is linear on $D_{0}-D_{1}$. A solution to (3.18) will be found having the form $\eta=\eta^{0}+\mathcal{D}^{\dagger} u$, where $\eta^{0}$ is a smooth section of $\sigma^{*} T X$ over $D_{0}$ which is annihilated by $\mathcal{D}$, and where $\mathcal{D}^{\dagger}$ is the formal $L^{2}$ adjoint of $\mathcal{D}$. Also, $u$ is a section of $\sigma^{*} T X \otimes T^{0,1} D$ which is constrained to vanish on the boundary of the closure of $D_{0}$. With $\eta^{0}$ fixed and with small norm (suitably measured), $u$ can be found as a fixed point of the map $\mathcal{T}$ which sends $u$ to

$$
\mathcal{T}(u)=-\left(\mathcal{D D}^{\dagger}\right)^{-1}\left(\kappa \mathcal{R}\left(\eta^{\prime}+\mathcal{D}^{\dagger} u, \nabla\left(\eta^{0}+\mathcal{D}^{\dagger} u\right)\right) .\right.
$$


Here, $\left(\mathcal{D D}^{\dagger}\right)^{-1}$ signifies the Dirichlet Green's function for $\mathcal{D D}^{\dagger}$. Standard techniques can be used to prove that (3.19) has a unique small solution $u$ provided that $\eta^{0}$ is small in an appropriate sense. In particular it is sufficient to measure $\eta^{0}$ with the norm $\|\cdot\|$ which is the sum of the $L_{1}^{2}$ norm over $D_{0}$ and the $C^{0,1 / 2}$ Holder norm over $D_{1}$. Indeed, a simple contraction mapping argument (as in the proof of Lemma 5.5 in [20] or [21]) finds $\delta>0$ and $\xi \geq 1$ such that when $\|\eta\|<\delta$, then (3.19) has a unique solution $u$ which obeys the constraint $\|u\|+\|\nabla u\| \leq \xi^{-1} \delta$. Infact, the solution obeys

$$
\|u\|+\|\nabla u\| \leq \xi\left\|\eta^{0}\right\|^{2} .
$$

Moreover, the solution $u$ varies smoothly with varying $\eta^{0}$. (The estimates which prove that $\mathcal{T}$ is a contraction on a suitable ball are facilitated by the fact that the Green's function $\left(\mathcal{D} \mathcal{D}^{\dagger}\right)^{-1}$ has the same singular behavior as the Laplacian on the disk. An essentially identical contraction mapping argument with similar estimates can be found in the proof of Lemma 5.5 in [20] and [21].)

With $u$ and (3.20) understood, Lemma 3.7 follows from the assertion that there exists $\zeta \geq 1$ such that for each $z \in D^{\prime}$ and each $\left.\eta_{z} \in \sigma^{*} T X\right|_{z}$, there is a section $\eta^{0}$ of $\sigma^{*} T X$ over $D_{0}$ which restricts to $z$ as $\eta_{z}$, is annihilated by $\mathcal{D}$, and obeys $\left\|\eta^{0}\right\| \leq \zeta\left|\eta_{z}\right|$. The next two steps prove this assertion.

Step 2 The first part of the proof of the preceding assertion gives an affirmative answer to the following question: Given $z \in D^{\prime}$ and a point $\left.\eta_{z} \in \sigma^{*} T X\right|_{z}$, is there an element $\eta^{0}$ in the kernel of $\mathcal{D}$ over $D_{0}$ which obeys $\eta^{0}(z)=\eta_{z}$ ?

To find such a section $\eta^{0}$, consider fixing a point $y \in D_{-1}-D_{0}$ and a point $\eta_{y}$ in $\left.\left.\sigma^{*} T X\right|_{y} \otimes T^{0,1} D\right|_{y}$. Then, let $v$ denote the section of $\sigma^{*} T X$ over $D_{0}$ which is given by $\mathcal{D}^{\dagger}\left(\mathcal{D D}^{\dagger}\right)^{-1}(\cdot, y) \eta_{y}$, where, in this step, $\left(\mathcal{D D}^{\dagger}\right)^{-1}$ denote the Greens with Dirichlet boundary conditions on the boundary of the closure of $D_{-1}$. Note that $\mathcal{D} v=0$ on $D_{0}$.

Now, consider varying $y$ over a small ball in $D_{-1}-D_{0}$ and varying the corresponding $\eta_{y}$. Restricting the corresponding $v$ 's to the point $z$ generates a vector subspace $\left.V \subset \sigma^{*} T X\right|_{z}$. The claim is that $V=\left.\sigma^{*} T X\right|_{z}$. Accept this claim and it follows that there is an element in the kernel of $\mathcal{D}$ over $D_{0}$ which restricts to $z$ as $\eta_{z}$.

Here is the proof of the claim: Were the claim false, there would exist a non-zero element $\left.\eta^{\prime} \in \sigma^{*} T X\right|_{z}$ with the property that $\eta^{\prime \dagger}\left(\mathcal{D D D}^{\dagger}\right)^{-1}(z, y)=0$ at all points $y$ from some nonempty, open subset of $D_{-1}-D_{0}$. Since $z \in D_{1}$, the section $\eta^{\prime \dagger}\left(\mathcal{D} \mathcal{D}^{\dagger}\right)^{-1}(z, \cdot)$ of $\sigma^{*} T X \otimes T^{0,1} D$ is annihilated by $\mathcal{D}^{\dagger}$ over $D_{-1}-D_{0}$. If it vanishes on a non-empty open set, Aronszajn's unique continuation theorem [1] 
forces it to vanish everywhere. Such is not the case. (Consider a point very close to $\mathrm{z}$ where the small distance asymptotics of $\left(\mathcal{D D}^{\dagger}\right)^{-1}(z, \cdot)$ can be invoked.)

Step 3 The previous step established the following: Given $z \in D^{\prime}$ there exists a set $\left\{\left(z_{j}, \eta_{j}\right)\right\}_{1 \leq j \leq 4}$ of four pairs consisting of points $\left\{z_{j}\right\} \subset D_{-1}-D_{0}$ and for each $z_{j}$, a corresponding non-zero element $\eta_{j}$ in the fiber of $\sigma^{*} T X \otimes T^{0,1} D$ at $z_{j}$. This set has the following property: The set $\left\{\mathcal{D}^{\dagger}\left(\mathcal{D D}^{t}\right)^{-1}\left(z, z_{j}\right) \eta_{j}\right\}_{1 \leq j \leq 4}$ of vectors in $\left.\sigma^{*} T X\right|_{z}$ spans $\left.\sigma^{*} T X\right|_{z}$. Now, because the spanning condition is an open condition, one can additionally conclude that $\left\{\mathcal{D}^{\dagger}\left(\mathcal{D D}^{t}\right)^{-1}\left(z^{\prime}, z_{j}\right) \eta_{j}\right\}_{1 \leq j \leq 4}$ also spans $\left.\sigma^{*} T X\right|_{z^{\prime}}$ for all points $z^{\prime}$ in some open neighborhood of $D$.

With the preceding understood, the fact that the closure of $D^{\prime}$ is compact implies that there exists a finite $N(\geq 4)$ and a set $\left\{\left(z_{j}, \eta_{j}\right)\right\}_{1 \leq j \leq N}$ with $\left\{z_{j}\right\} \subset$ $D_{-1}-D_{0}$ and with $\eta_{j}$ in the fiber of $\sigma^{*} T X \otimes T^{0,1} D$ at $z_{j}$ and such that $\left\{\mathcal{D}^{\dagger}\left(\mathcal{D D}^{t}\right)^{-1}\left(z, z_{j}\right) \eta_{j}\right\}_{1 \leq j \leq N}$ spans $\left.\sigma^{*} T X\right|_{z}$ at each point of $D^{\prime}$.

The fact that $D^{\prime}$ has compact closure in $D_{0}$, and the fact that the integer $N$ here is finite imply the following: There exists $\zeta \geq 1$ such that for each point $z \in D^{\prime}$ and $\left.\eta_{z} \in \sigma^{*} T X\right|_{z}$, there exists a section, $\eta^{0}$, of $\sigma^{*} T X$ over $D_{0}$ which obeys $\eta^{0}(z)=\eta_{z}, \mathcal{D} \eta^{0}=0$ and $\|\eta\| \leq \zeta\left|\eta_{z}\right|$. Given this last remark, the conclusions of Step 1 above can be brought to bear to finish the proof of Lemma 3.7.

Before ending this section, it is pertinent to remark here that the proof of Proposition 3.3 just offered proves, with minimal modifications, a somewhat stronger local compactness theorem. This stronger version of Proposition 3.3 is not necessary for this article, but is stated below for possible future reference:

Proposition 3.8 Let $Y$ be a smooth 4-manifold with symplectic form $\omega$ and a compatible almost complex structure $J$. Let $\left\{Y_{s}\right\}_{s \rightarrow 0}$ be a countable exhaustion of $Y$ by a nested sequence of open sets (so $Y_{s} \subset Y_{s^{\prime}}$ with compact closure when $\left.s>s^{\prime}\right)$. In addition, suppose that $\left\{\left(\omega_{s}, J_{s}\right)\right\}_{s \rightarrow 0}$ is a corresponding sequence whose typical element is a pair consisting of a symplectic form, $\omega_{s}$, on $Y_{s}$, and a compatible almost complex structure $J_{s}$. Require that $\left.\left\{\omega_{s}, J_{s}\right)\right\}_{s \rightarrow 0}$ converges to $(\omega, J)$ in the $C^{\infty}$ topology on compact subsets of $Y$. Next, let $E \geq 1$ be given with a sequence $\left\{C_{s} \subset Y_{s}\right\}_{s \rightarrow 0}$ where $C_{s}$ is a finite energy, $J_{s}-$ pseudo-holomorphic subvariety with $\int_{C_{s}} \omega_{s} \leq E$. Then there is a $J$-pseudoholomorphic subvariety $C_{0} \subset Y$ and a subsequence of $\left\{C_{s}\right\}$ (hence renumbered consecutively from 1) with the following properties:

- The integral of $\omega$ over $C_{0}$ is bounded also by $E$.

- $\left\{C_{s}\right\}$ converges geometrically to $C$. 
Proof of Proposition 3.8 The only essential modification of the proof of Proposition 3.3 occurs in (3.18) and (3.19) with the addition of an $s$-dependent term $\mathcal{R}_{s}$ to $\mathcal{R}$ which is $\mathbb{R}$-linear in $\eta$ and accounts for the difference between $J$ and $J_{s}$. However, as $J-J_{s}$ converges to zero on compact subsets of $Y$, this term has no substantive affect on the subsequent arguments.

\section{The multiplicity of the limit}

Fix $t_{0} \in Z$. Then the constructions of the previous section find a convergent subsequence in the ball $B(1)$ from the sequence $\left\{C_{s}\right\}_{s>0}$ of rescaled versions of the original subvariety $C$. As currents, the subsequence of $\left\{C_{s}\right\}_{s>0}$ converges to a current in $B(1)$. This current can be written as a sum of currents with integer multiplicities, where each term corresponds to one of the cases from Examples 1.3, 1.4 and 1.5. The following proposition makes a somewhat stronger statement; for it asserts, in part, that all convergent subsequences of $\left\{C_{s}\right\}_{s>0}$ have the same limit current.

Proposition 4.1 Let $C$ be a finite energy, pseudo-holomorphic subvariety. Fix a point $t_{0} \in Z$. Let $B$ denote the ball of radius 1 in $S^{1} \times \mathbb{R}^{3}$ with center $t_{0}$. For $s>0$, but small, introduce the sequence $\left\{C_{s}\right\}$ of pseudo-holomorphic subvarieties of $B$ as defined in (3.1). Then, as a sequence of currents, every limit of $\left\{C_{s}\right\}_{s>0}$ has the form

$$
\sum_{1 \leq i \leq p} C_{\rho}^{(i)}+q_{+} \cdot C_{z+}+q_{-} \cdot C_{z-}+\sum_{1 \leq i \leq n_{+}} C_{h+}^{(i)}+\sum_{1 \leq i \leq n_{-}} C_{h-}^{(i)} .
$$

Here, $\left\{C_{\rho}^{(i)}\right\}$ is a finite set of (not necessarily distinct) pseudo-holomorphic submanifolds which are described in Example 1.3 (with $\nu$ determined by the index $i$ ); $C_{z \pm}$ are described by Example 1.4 (the \pm signifies when $\nu=(0,0, \pm 1)$ ) while $q_{ \pm}$are non-negative integers; and $\left\{C_{h \pm}^{(i)}\right\}$ is a finite set of (not necessarily distinct) pseudo-holomorphic submanifolds with are described in Example 1.5 where the constant $c$ is determined by the index $i$ while the \pm correlates with the signs used in (1.7). Furthermore, the integers $p, q_{+}, q_{-}, n_{+}, n_{-}$are identical for any limit; and the corresponding sets $\left\{c^{(i)}\right\} \subset\left(0,2 / 3^{3 / 2}\right]$ which determine (in part) the Example 1.5 data $\left\{C_{h \pm}^{(i)}\right\}$ are identical for any limit up to permutations.

This proposition implies that the limiting data in (4.1) is unique except possibly up to separate rotations $\left(\phi \rightarrow \phi+\right.$ constant) of each element in $\left\{C_{\rho}^{(i)}\right\}$ and $\left\{C_{h \pm}^{(i)}\right\}$. 
The remainder of this section is occupied with the proof of Proposition 4.1.

Proof of Proposition 4.1 The fact that all possible limit currents are given by a formal sum of the form in (4.1) (with all coefficients non-negative) is a restatement of Proposition 3.2. At issue here is the assertion that there is a unique limit. The proof of this assertion is broken into four steps.

Step 1 This step verifies the assertion that $p-q$ is uniquely defined and independent of $t_{0}$. Indeed, this assertion follows from the observation that $p-q$ is the intersection number between $C$ and a 2 -sphere of the form $t=$ constant, $\rho^{2}+z^{2}=\delta>0$. This can be seen from the following argument: Let $S$ be the sphere $t=t_{0}$ and $\rho^{2}+z^{2}=1 / 2$. This sphere has trivial normal bundle, so one can identify a tubular neighborhood of $S$ with the product $S \times D$, where $D$ is small radius disk in the plane. (Here, the sphere $S$ is identified with $S \times 0 \subset S \times D$.) Next, fix a compactly supported, non-negative 2-form on $D$ with integral equal to 1 . This form defines a closed 2 -form, $\mu$, on $S \times D$ which pairs with the current in (4.1) to give $p-q$. (All of the cases of Example 1.5 have intersection number zero with a $t=$ constant 2 -sphere.) Thus, with $\varepsilon>0$ fixed, and $s$ large, this $\mu$ pairs with $C_{s}$ from the subsequence to give a number, say $y_{s}$, which is within $\varepsilon$ of $p-q$.

However, $\mu$ is closed, and thus $y_{s}$ is equal to the pairing between $C_{s}$ and any 2 -form $\mu^{\prime}=\mu+d \nu$, where $\nu$ is a 1 -form with compact support in $S \times$ $D$. In particular, fix $s$ large, and perturb $S$ slightly (if necessary) so that its intersection with $C_{s}$ is transversal. Then one can find a 2-form $\mu^{\prime}$ with support in any tubular neighborhood of this perturbation (and thus in $S \times D$ ) whose integral over $C_{s}$ is equal to the intersection number of $C_{s}$ with the perturbed version of $S$.

With the preceding understood, it follows that this intersection number is within $\varepsilon$ of $p-q$, and thus equal to $p-q$ as both numbers are integers. Finally, rescaling shows that this intersection is the same as that between $C$ and a perturbation of $t=t_{0}$, and $\rho^{2}+z^{2}=s^{2} / 2$ sphere. Finally, remark that the intersection between $C$ and such a sphere is the same as that between $C$ and any tiny perturbation of the sphere $t=t_{0}, \rho^{2}+z^{2}=1 / 2$, as these two spheres are homologous in the complement of $Z$. In particular, this shows that the difference, $p-q$, is independent of the chosen subsequence of $\left\{C_{s}\right\}$.

The same invariance under perturbation argument shows that $p-q$ is also independent of $t_{0}$.

Step 2 Let $\Omega \subset(0,1]$ be a countable, decreasing set with limit zero and such that the sequence $\left\{C_{s}\right\}_{s \in \Omega}$ converges as described in Proposition 3.2. The 
limit in (4.1) determines, among other things, the integers $p$ and $q_{ \pm}$. This step identifies these integers as intersection numbers with the following claim: There exists $d_{0}>0$ such that when $\delta$ is positive and less than $\delta_{0}$, then:

- $p$ is the intersection number in $B(1)$ between $C_{s}$ for small $s$ and the pseudo-holomorphic cylinder $t=t_{0}, f=1 / 100$ and $|z|<\delta$.

- $q_{ \pm}$is the intersection number in $B(1)$ between $C_{s}$ for small $s$ and the pseudo-holomorphic disk $t=t_{0}, \quad f=-1 / 100, \rho<\delta$ and $\pm z>0$. (4.2)

The remainder of this step offers a proof of the claim for $q_{+}$. The proofs for $p$ and $q_{-}$are completely analogous and will be omitted.

To begin the argument, consider that $\rho$ is bounded from below on a case from Example 1.5 by $s \cdot c$. Thus, since only finitely many of the Example 1.5 cases appear in (4.1), there exists $\delta_{0}>0$ such that each $C_{h+}^{(i)}$ which appears in (4.1) has empty intersection in $B(1)$ with the disk $t=t_{0}, f=-1 / 100, \rho<\delta_{0}$ and $z>0$. With the preceding understood, take $\delta<\delta_{0} / 4$ and let $\Sigma$ denote the $t=t_{0}, f=-1 / 100, \rho<\delta$ and $z>0$ disk. Let $D \subset \mathbb{C}$ denote the standard disk, and fix an identification of a tubular neighborhood of $\Sigma$ in $S^{1} \times B^{3}$ with $\Sigma \times D$ so that $\Sigma$ in the former is identified with $\Sigma \times\{0\}$ in the latter. Also, arrange the identification so that the functions $\phi$ and $h$ are constant on each $\{$ point $\} \times D \subset \Sigma \times D$. Finally, require $f<0$ on this tubular neighborhood. Now, given $\varepsilon>0$, let $\mu_{\varepsilon}$ denote a smooth 2 -form on $D$ with integral 1 and support in the concentric disk $D_{\varepsilon} \subset D$ of radius $\varepsilon$. Modify $\mu_{\varepsilon}$ (via multiplication by an $\varepsilon$-independent, compactly supported function on $\Sigma$ ) so that the result, $\mu_{\varepsilon}^{\prime}$, has compact support in $S^{1} \times\left(B^{3}-\{0\}\right)$ and also agrees with $\mu_{\varepsilon}$ on some $\varepsilon$-independent neighborhood the $h=0$ disk in $\Sigma \times D$. This last condition insures that $\mu_{\varepsilon}^{\prime}$ is closed near the $h=0$ disk in $\Sigma \times D$.

By definition, the current in (4.1) pairs with $\mu_{\varepsilon}^{\prime}$ to give the integer $q_{+}$. It then follows that the current $C_{s}$ for $s \in \Omega$ and small will pair with each $\mu_{\varepsilon}^{\prime}$ to give $q_{+}$also. Since $\varepsilon$ can be arbitrarily small here (though positive), and $\mu_{\varepsilon}^{\prime}$ is closed near the $h=0$ disk in $\Sigma \times D$, the perturbation argument from Step 1 can be applied here to prove that $C_{s}$ has intersection number $q_{+}$with $\Sigma$ for small $s$.

Step 3 This step argues that $p, q_{+}$and $q_{-}$are the same for any limit of the sequence $\left\{C_{s}\right\}$. Again, the details will be given for the case of $q_{+}$with the analogous arguments for $q_{-}$and $p$ left to the reader. (In fact, once $q_{ \pm}$are known to be unique, then the uniqueness of $p$ follows from Step 1.)

To begin the discussion, suppose, for argument's sake, that there exist decreasing sequences $\Omega, \Omega^{\prime} \subset[0,1]$, both having limit zero and such that the 
corresponding sequences $\left\{C_{s}\right\}_{s \in \Omega}$ and $\left\{C_{s}\right\}_{s \in \Omega^{\prime}}$ converge geometrically to limits given by (4.1) with the corresponding $q_{+}$and $q_{+}^{\prime}$ not equal. A contradiction will follow from this assumption.

To obtain the contradiction, reintroduce, for $\delta>0$ and very small, the pseudoholomorphic disk $\Sigma$ where $t=t_{0}, f=-1 / 100, \rho<\delta$ and $z>0$. Then, use (4.2) to conclude that when $\delta$ is small, then $C_{s}$ for all small $s \in \Omega$ has intersection $q_{+}$with this $\Sigma$. Likewise, one can choose $\delta$ still smaller if necessary so that $C_{s}$ for all small $s^{\prime} \in \Omega$ has intersection number $q_{+}^{\prime}$ with $\Sigma$. Moreover, for sufficiently small $s$, these intersection points in either case will lie where $\rho$ is less than any given positive constant. In particular, one can find a small number $c>0$ such that the following is true:

- The period of the corresponding solution to (1.6) is an irrational multiple of $\pi$.

- The level set $h /\left(\rho^{2}+z^{2}\right)^{3 / 2}=c$ intersects $\Sigma$ as a circle.

- For sufficiently small $s$ in either $\Omega$ or $\Omega^{\prime}$, all intersection points of $C_{s}$ with $\Sigma$ lie where $h /\left(\rho^{2}+z^{2}\right)^{3 / 2}<c$.

(See Lemma 1.9 about the first point.)

Now remember that $C_{s}$ is obtained from $s$ by rescaling, so that the intersections between $C_{s}$ and $\Sigma$ are obtained by rescaling those between $C$ and $\Sigma_{1 / s}$, where $\Sigma_{1 / s}$ has $t=t_{0}, f=-s^{2} / 100, \rho<s \delta$ and $z>0$.

With the preceding understood, consider the following construction: To any given $s \in \Omega$, let $s^{\prime}(s) \in \Omega^{\prime}$ denote the largest element which is less than $s$. For $\sigma \in\left[s^{\prime}(s), s\right]$, consider the intersection of $C$ with $\Sigma_{1 / s}$. For $\sigma$ near $s$, the intersection number is $q_{+}$and all intersection points have $h /\left(\rho^{2}+z^{2}\right)^{3 / 2}<c$. Likewise, for $\sigma$ near $s^{\prime}$, the corresponding intersection number is $q_{+}^{\prime}$ and all intersection points also have $h /\left(\rho^{2}+z^{2}\right)^{3 / 2}<c$. Since intersection number is a deformation invariant (for compactly supported homotopies), it follows that there exists $s^{\prime \prime} \in\left(s^{\prime}(s), s\right)$ where $C$ has an intersection with $\Sigma_{1 / s^{\prime \prime}}$ which lies where $h /\left(\rho^{2}+z^{2}\right)^{3 / 2}=c$.

This last conclusion implies that there is a sequence $\Omega^{\prime \prime} \subset[0,1]$ which decreases to zero and which has the property that each $C_{s}$ for $s \in \Omega^{\prime \prime}$ intersects the $h /\left(\rho^{2}+z^{2}\right)^{3 / 2}=c$ set. According to Proposition 3.2, this sequence has a convergent subsequence with a geometric limit which is a union of cases from Examples 1.3, 1.4 and 1.5. This limit must include a case from Example 1.5 which is determined by the given value of $c$. However, as this value of $c$ corresponds to a solution to (1.6) with whose period is not in $\pi \mathbb{Q}$, the corresponding 
pseudo-holomorphic submanifold will not have finite energy. This is the contradiction which establishes that $q_{+}=q_{+}^{\prime}$.

Step 4 Each limit as in (4.1) determines a set $\left\{C_{h \pm}^{(i)}\right\}$ of pseudo-holomorphic submanifolds which are described by Example 1.5. The latter determines a corresponding set of constants $\left\{c^{(i \pm)}\right\} \subset\left(0,2 / 3^{3 / 2}\right]$. (Note that this set need not consist of distinct elements.) The purpose of this step is to establish that the set $\left\{c^{(i \pm)}\right\}$ does not depend on chosen subsequence. This is to say that all limits of $\left\{C_{s}\right\}$ determine the same set $\left\{c^{(i \pm)}\right\}$ up to permutations.

What follows is the argument for $\left\{c^{(i+)}\right\}$. The argument for $\left\{c^{(i-)}\right\}$ is completely analogous and will be omitted. To start the argument suppose that $c \in\left(0,2 / 3^{3 / 2}\right]$ is such that the corresponding solution to (1.6) has period which is a rational multiple of $\pi$. This $c$ determines a least positive integer, $b(c)$, for which the corresponding solution to $(1.6)$ is periodic on $[0,2 \pi b]$. Extend this assignment $b(\cdot)$ to a function on $\left(0,2 / 3^{3 / 2}\right]$ by declaring that $b(c)=\infty$ when the period in question is an irrational multiple of $\pi$. With the preceding understood, the assertion that the set $\left\{c^{(i+)}\right\}$ is limit independent is equivalent to the assertion that $\sum_{c^{(i+)}>c} b\left(c^{(i+)}\right)$ is limit independent for each $c \in\left(0,2 / 3^{3 / 2}\right]$ with $b(c)=\infty$.

To establish the latter claim, fix $c$ with $b(c)=\infty$ and argue as in Steps 1 and 2 above to prove that

- $\sum_{c^{(i+)}>c} b\left(c^{(i+)}\right)$ is the intersection number in the ball $B(1)$ between $C_{s}$ for $s \in \Omega$ sufficiently small and the pseudo-holomorphic submanifold where $\phi=0$ and $h=c$.

Then, by rescaling, (4.4) is the same as the assertion that $\sum_{c^{(i+)}>c} b\left(c^{(i+)}\right)$ is the intersection number in the ball $B(s)$ (for $s \in \Omega$ ) between $C$ and the pseudo-holomorphic submanifold where $\phi=0$ and $h=c s^{3}$.

Given the preceding, suppose that $\Omega$ and $\Omega^{\prime}$ are a pair of countable, decreasing subsets of $[0,1]$ with limit zero and such that the corresponding sequences $\left\{C_{s}\right\}_{s \in \Omega}$ and $\left\{C_{s}\right\}_{s \in \Omega^{\prime}}$ converge as described in (4.1) and to yield the data $\left\{c^{(i+)}\right\}$ and $\left\{c^{\prime(i+)}\right\}$, respectively. Suppose that the sum $\sum_{c^{(i+)}>c} b\left(c^{(i+)}\right)$ differs from its primed analog. This supposition leads to a contradiction as follows: By an argument which is completely analogous to that used in Step 3 above, one can construct, for each $s \in \Omega$, a number $s^{\prime \prime} \equiv s^{\prime \prime}(s) \in(0, s)$ with the property that $C$ intersects the pseudo-holomorphic submanifold where $\phi=0$ and $h=c s^{\prime \prime} 3$ on the boundary of the ball $B\left(s^{\prime \prime}\right)$. The corresponding sequence $\Omega^{\prime \prime} \equiv\left\{s^{\prime \prime}(s)\right.$ : $s \in \Omega\}$ would then converge geometrically as described in Proposition 3.2 to 
a limit which is given by (4.1). However, for this limit, the corresponding set $\left\{c_{h}^{\prime \prime}{ }_{h}^{(i+)}\right\}$ would contain an element which has $h=c$ on the boundary of the ball $B(1)$. The corresponding $c \in\left(0,2 / 3^{3 / 2}\right]$ for such an element has $b(c)=\infty$ and thus, the corresponding solutions to (1.6) do not have periods in $\pi \mathbb{Q}$. As these values for $c$ are not allowed in Example 1.5 (since they yield infinite energy pseudo-holomorphic varieties), the desired contradiction has been obtained.

\section{$5 \quad$ The integers $n_{ \pm}$}

The previous section associates to each $t_{0} \in Z$ the ordered set $\left(p, q_{+}, q_{-}, n_{+}, n_{-}\right)$ of non-negative integers. The purpose of this section is to prove:

Proposition 5.1 There are only finitely many $t_{0} \in Z$ where $n=n_{+}+n_{-} \neq 0$.

This proposition is a corollary to a second proposition of some independent interest. The statement of this second proposition requires a brief digression. To start the digression, fix $\delta \in[1 / 16,1 / 8]$ and, for small $s>0$, consider the compact set $\Omega(s)$ in $B^{3}$ where

$$
|h| \leq s^{3}, \quad \rho \leq \sqrt{\delta} \text { and }|z| \leq \delta .
$$

Thus, the boundary of $\Omega(s)$ consists of the parts:

(1) $h= \pm s^{3}$ and $\rho \leq \sqrt{\delta}$ and $|z| \leq \delta$

(2) $\rho=\sqrt{\delta}$ and $|z| \leq s^{3} / \delta$

(3) $|z|=\delta$ and $\rho \leq s^{3 / 2} / \sqrt{\delta}$

Use $C(s)$ to denote $C \cap\left(S^{1} \times \Omega(s)\right)$. With $\delta$ fixed, introduce the following function of $s$ :

$$
\mu(s)=\int_{C(s)} d \phi \wedge d h
$$

Now, end the digression and consider the following proposition:

Proposition 5.2 There is a constant $\zeta>0$ such that $\mu(s) \leq \zeta s^{3}$ for all $s$ sufficiently small.

Note that this proposition has corollary which bounds the integral of $d \phi \wedge d h$ over the set $C^{\prime}(r)=C \cap\left(S^{1} \times\left\{\rho^{2}+z^{2} \leq r\right\}\right)$ by an $r$-dependent multiple of $r^{3}$. That is, there exists $\zeta>0$ which is independent of $r$ and is such that

$$
\int_{C^{\prime}(r)} d \phi \wedge d h \leq \zeta r^{3}
$$


for all $r<1$. (This bound follows from Proposition 5.2 because the ball of radius $r$ is contained in $\Omega\left(s=2^{1 / 2} / 3^{1 / 2} r\right)$.)

Proof of Proposition 5.1 assuming Proposition 5.2 The first point to make is that the form $d \phi \wedge d h$ restricts to $C$ as a non-negative multiple of $\omega$, and thus $\mu(s)$ is no smaller than the integral of $d \phi \wedge d h$ over any subset of $C$ in $C(s)$. With this understood, the plan is bound $\mu(s)$ from below by considering the restriction of the integral in question to the intersection of $C$ with small balls about the points in $Z_{0}$ where $n \neq 0$.

However, for this purpose, it is necessary to digress with a return to Example 1.5. To start the digression, suppose that $\Sigma$ is given by Example 1.5. Let $b$ denote the positive integer supplied in the example. (This integer is the smallest $b^{\prime} \in\{1,2, \ldots\}$ such that the solutions to (1.6) are periodic on $\left.\left[0,2 \pi b^{\prime}\right].\right)$ With $s>0$ given, note that set of points on $\Sigma$ where $|h| \leq s^{3}$ is contained in the ball of radius $s / c^{1 / 3}$ and center at 0 . (Here, $c$ is the constant in $\left(0,2 / 3^{3 / 2}\right]$ which determines $\Sigma$ up to rotations in the $x-y$ plane.) In particular, the set where $|h| \leq s^{3}$ on $\Sigma$ is compact and Stoke's theorem finds

$$
\int_{\Sigma \cap\{h \mid \leq s\}} d \phi \wedge d h=2 \pi b s^{3} \geq 2 \pi s^{3}
$$

End the digression. With the preceding understood, suppose that the set of points in $Z$ where $n \neq 0$ contains at least $N$ points, where $N$ is some given integer. Then, it follows from (5.3) and Propositions 3.2 and 4.1 that $\mu(s) \geq$ $2 \pi N s^{3}$ when $s$ is small. This last lower bound for $\mu$ plus Proposition 5.2 bounds $N$.

Proof of Proposition 5.2 The existence of a bound $s^{-3} \cdot \mu(s) \leq \zeta$ is argued as follows: First of all, one can remove from $C$ all irreducible components which are described by cases in Examples 1.3 and 1.4 since these make no contribution to $\mu(s)$.

Next, note that the intersection of $C$ with the surfaces $\rho=\sqrt{\delta}$ and $|z|=\delta$ defines a smooth curve for all but finitely many $\delta$ in $[1 / 16,1 / 8]$. This follows from Sard's theorem. Likewise, the intersection of $C$ with the surface $|h|=s^{3}$ is a smooth curve for all but countably many values of $s$ in $(0,1]$. Thus, with $\delta$ chosen appropriately, and with $s$ chosen from the complement of a countable set, the number $\mu(s)$ can be computed via Stokes' theorem as

$$
\begin{aligned}
\mu(s)=-s^{3} \cdot \int_{C(s) \cap\{h=s\}} d \phi+s^{3} \cdot \int_{C^{\prime}(s) \cap\{h=-s\}} d \phi & \text { (5.6) } \\
& -\int_{C^{\prime}(s) \cap\{\rho=\sqrt{\delta}\}} h \cdot d \phi-\int_{C^{\prime}(s) \cap\{|z|=\delta\}} h \cdot d \phi .
\end{aligned}
$$


(The integration by parts formula here can be justified by employing the functions $\left\{\eta_{\varepsilon}\right\}$ as in (2.4). This step is straightforward and is left to the reader.)

Consider now the various terms in (5.6). As $C$ does not coincide with the product of $S^{1}$ and either the positive or negative $z$-axis, then for sufficiently small $s$, the right most term in (5.6) will be zero for all but finitely many choices of $\delta$.

To understand the contribution to $\mu(s)$ from the term which is second from the right in (5.6), remark first that $|d \phi|=\rho^{-1}$, so the restriction of $d \phi$ to $C \cap\left\{\rho=\delta^{1 / 2},|z| \leq s / \delta\right\}$ has norm less than or equal to $\delta^{-1 / 2}$. Meanwhile, on this part of $C$, one has $h=z \cdot \delta$. Finally, this part of $C$ is uniformly far from $Z$ (since $\delta \geq 1 / 4$ ), and so the length of this part of $C$ is bounded independently of $s$. With the preceding understood, it follows that the term which is second from the right in (5.6) has absolute value no greater than $\zeta \cdot s^{3}$, where $\zeta$ is a constant which depends on $C$, but not on $s$.

To analyze the left most term in (5.6), consider completing the integration path $\nu=C \cap\left\{h=s^{3}, \rho \leq \sqrt{\delta}, z \leq \delta\right\}$ to make a closed path in $C$ which avoids where $\rho=0$ and which stays uniformly away from the set $Z$. For example, one could add to $\nu$ the following:

$$
\nu^{\prime}=C \cap\left\{\rho^{4}+z^{2}=\delta^{2}+s^{3} / \delta^{2}, s^{3 / 2} / \delta \leq \rho, s^{3} / \delta \leq z\right\}
$$

or any slight perturbation thereof. For generic $s$, this last is a smooth curve in $C$ whose distance from $Z$ is at least $\sqrt{\delta}$. Thus, the integral of $d \phi$ over $\nu^{\prime}$ is uniformly bounded, independent of $s$. Furthermore, the curve $\nu^{\prime}$ is homologous in $C-C \cap \rho^{-1}(0)$ to $\nu$ since both are obtained by slicing $C$ with functions which are defined on $B^{3}-\rho^{-1}(0)$. (Consider restricting the family of functions $\left\{w_{t}=t \cdot\left(\rho^{4}+z^{2}-\delta^{2}-s^{6} / \delta^{2}\right)+(1-t) \cdot\left(\rho^{2} z-s^{3}\right)\right\}_{t \in[0,1]}$ to the set to $C \cap\left\{s^{3 / 2} / \sqrt{\delta} \leq\right.$ $\left.\rho, s^{3} / \delta \leq z\right\}$. Then, the family $\left\{w_{t}^{-1}(0)\right\}_{t \in[0,1]}$ defines a homology rel endpoints between $\nu$ and $\nu^{\prime}$ in $C-C \cap \rho^{-1}(0)$.)

Since $\nu$ and $\nu^{\prime}$ are homologous rel end points in $C \cap \rho^{-1}(0)$, the integral of $d \phi$ over $\nu$ is also uniformly bounded, independent of $s$. Thus, the left-most term in (5.6) has absolute value no greater than $\zeta \cdot s^{3}$, where $\zeta$ depends on $C$, but not on $s$ when the latter is small.

The term which is second to the left in (5.6) is handled in the same way as the previous term.

It follows from the preceding that $\mu(s) \leq \zeta \cdot s^{3}$, where $\zeta$ depends on $C$, but is independent of $s$, as was required. Note that this estimate is independent of the choice of $\delta$ as well since with s fixed, $\mu(s)$ is a continuous, increasing function of $\delta$. 


\section{Semi-continuity of $p$ and $q$ as functions on $Z$}

For each point $t_{0} \in Z$, Proposition 4.1 assigns the ordered pair of non-negative integers $\left(p, q=q_{+}+q_{-}\right)$. That is, $(p, q)$ from Proposition 4.1 can be thought of as a pair of functions on $Z$. The purpose of this section is to investigate $(p, q)$ as functions on $Z$.

Before starting, remark that there are only a finite number of points in $Z$ where the integer $n$ in (4.1) is non-zero. It follows from the convergence properties as described in Proposition 3.2 that there exists $r_{0}>0$ such that $C \cap\left\{\rho^{2}+z^{2} \leq\right.$ $\left.r_{0}^{2}\right\}$ can be decomposed as the disjoint union $C^{\prime} \cup C^{\prime \prime}$ of pseudo-holomorphic subvarieties with the following properties: All points in $Z$ have $n \equiv n_{+}+n_{-}=0$ when the latter is computed using $C^{\prime}$ instead of $C$. Conversely, all points in $Z$ have $p=q=0$ when these integers are computed using $C^{\prime \prime}$ instead of $C$. With this understood, it follows that no generality is lost by considering solely the case where $C=C^{\prime}$. This condition will henceforth be assumed.

In addition, one can assume with out loss of generality that $C \neq C_{1} \cup C_{2}$ where $C_{2}$ is described in either Example 1.3 or 1.4.

Consider now a sequence $\left\{t_{i}\right\} \subset Z$ which converges to some $t_{0} \in Z$. As there is a uniform bound on the values for $p$ and $q$ over the whole of $Z$ (from Proposition 2.1), one can assume, without loss of generality, that $p$ and $q$ are constant on the sequence $\left\{t_{i}\right\}$. Use $\left(p_{1}, q_{1}\right)$ to denote this constant value. The goal in this section is to prove the following proposition:

Proposition 6.1 Define $\left(p_{0}, q_{0}\right)$ and $\left(p_{1}, q_{1}\right)$ as above. Then $p_{0} \geq p_{1}$ and $q_{0} \geq q_{1}$.

(Remember from the proof of Proposition 4.1 that $p_{0}-q_{0}=p_{1}-q_{1}$. )

The proof of Proposition 6.1 depends on Lemma 6.2, below. The statement of the latter requires the introduction, for $t^{\prime} \in Z$ and $s>0$, of the pseudoholomorphic submanifold $\Sigma\left(t^{\prime}, s\right) \subset X$ which is defined by the equations $t=t^{\prime}$ and $f=s^{2}$.

Lemma 6.2 There exists $\delta, \varepsilon>0$ such that the following is true: If $s>0$ but small, and if $t^{\prime} \in Z$ obeys $\left|t_{0}-t^{\prime}\right|<\varepsilon$, then:

(1) The intersection of $\Sigma\left(t^{\prime}, s\right)$ with $C \cap B(\delta)$ is contained in $C \cap B(\delta / 2)$.

(2) The integer $p_{0}$ is equal to the algebraic intersection number between $C \cap B(\delta)$ and any sufficiently small perturbation of $\Sigma\left(t^{\prime}, s\right)$. 
Remark that when $C$ and $\Sigma\left(t^{\prime}, s\right)$ are transverse, the algebraic and geometric intersection numbers agree since both $C$ and $\Sigma$ are pseudo-holomorphic.

Proof of Proposition 6.1 Accept Lemma 6.2 for the moment. Fix the index $i$ large, and consider the interesection number between $C \cap B(\delta)$ and $S\left(t_{i}, s\right)$ as $s$ tends to zero. These intersections will still be in the ball $B(\delta / 2)$, so the algebraic intersection number here is still $p_{0}$. To be precise, if $\Sigma\left(t_{i}, s\right)$ is not transversal to $C$ in $B(\delta)$, then make any arbitrarily small perturbation of the former to make a submanifold $\Sigma^{\prime}$ which is transversal to $C$ in $B(\delta)$. Then, the intersection number of $\Sigma^{\prime}$ with $C \cap B(\delta)$ will equal $p_{0}$. Furthermore, using the analysis of Lemma 5.5 in [20], one can find such small perturbations for which $\Sigma^{\prime}$ is pseudo-holomorpic near its intersection points with $C$. In this case, $\Sigma^{\prime}$ will intersect $C$ in precisely $p_{0}$ points because all points of intersection must have positive local sign.

Now, remark that for $s$ tiny, the algebraic intersection number of $\Sigma\left(t_{i}, s\right)$ with $C$ in a very small ball about $t_{i}$ must equal $p_{1}$. Indeed, this is simply Lemma 6.2 but with $t_{i}$ replacing $t_{0}$. In fact, since $C$ is pseudo-holomorphic, for $\mathrm{s}$ sufficiently small, any perturbation of $\Sigma\left(t_{i}, s\right)$ which is pseudo-holomorphic and transversal to $C$ in $B(\delta)$ and which is sufficiently close to $\Sigma\left(t_{j}, s\right)$ will intersect $C$ in exactly $p_{1}$ points (all with positive sign) in some very small ball about $t_{j}$. Since all other points of intersection with $C$ in $B(\delta)$ will also have positive sign, this forces the inequality $p_{1} \leq p_{0}$.

Proof of Lemma 6.2 Construct the sequence $\left\{C_{s}\right\}$ for the point $t_{0}$. For all $s$ sufficiently small, the current $C_{s}$ will be close to the current in (4.1) with $n_{+}=n_{-}=0, p=p_{0}$ and $q_{+}+q_{-}=q_{0}$. Furthermore, all points in $C_{s}$ will be very close to either the $z$-axis or the $x-y$ plane. Fix $t^{\prime} \in Z$ with $\left|t_{0}-t^{\prime}\right|<1 / 100$. Thus, for any value of $\varepsilon \in(0,1 / 100)$, all intersection points of $C_{s}$ with the submanifold $\Sigma\left(t^{\prime}, \varepsilon\right)$ are in $B(1 / 2)$ when $s$ is very large. (This is because $\Sigma$ exits $B(1 / 2)$ where $|\rho|^{2}+|z|^{2}=1 / 2$, and $2^{-1} \cdot \rho^{2}-z^{2}=\varepsilon$, which is far from where either $z$ or $\rho$ is zero.) Moreover, according to (4.2), $p_{0}$ is the intersection number between $C_{s}$ and $\Sigma(0, \varepsilon)$ when $s$ is large. Now, rescale the discussion back to $C$ to conclude that there exists $\delta>0$ such that when $t^{\prime} \in Z$ obeys $\left|t_{0}-t^{\prime}\right|<\delta / 100$, and when $s$ is sufficiently small, then the intersection between $C \cap B(\delta)$ and $\Sigma\left(t^{\prime}, s\right)$ is contained in the ball $B(\delta / 2)$ and the intersection number equals $p_{0}$. 


\section{$7 \quad$ Regular points}

In this section, suppose that $C$ is a pseudo-holomorphic subvariety with the property that all points in $Z$ have $n_{ \pm}=0$. With the preceding understood, call a point $t \in Z$ regular when $t$ is a local minimum of the function $p(\cdot)$. This is to say that $p(t) \leq p\left(t^{\prime}\right)$ for all $t^{\prime}$ sufficiently close to $t$. Let $Z_{R}$ denote the set of regular points in $Z$. The complement of $Z_{R}$ will be denoted by $Z_{S}$ and a point in $Z_{S}$ will be called singular.

The first observation here is that the set of regular points is open and dense in $Z$. The fact that $Z_{R}$ is open follows from Proposition 6.1 , and the fact that $Z_{R}$ is dense follows because $p(\cdot)$, being integer valued and bounded from below, takes on a local minimum on every open set. Note that $p(\cdot)$ is locally constant on $Z_{R}$ when considered as integer valued functions on $Z_{R}$.

The following proposition summarizes the behavior of $C$ near a regular point.

Proposition 7.1 The functions $p$ and $q_{ \pm}$(from (4.1)) are constant on $Z_{R}$, and the latter take values in $\{0,1\}$. Furthermore, given $t_{0} \in Z_{R}$, there exists $s_{0}>0$ with the following significance: Let $C_{*}$ denote a subset of $C$ where $t \in\left(t_{0}-s_{0}, t_{0}+s_{0}\right)$ and $\left(\rho^{2}+z^{2}\right)^{1 / 2}<s_{0}$. Then $C_{*}$ decomposes as the disjoint union $C_{*}=C_{\rho} \cup C_{z+} \cup C_{z-}$ where:

(1) If $q_{ \pm}=0$, then $C_{z \pm}$ is empty; otherwise $C_{z \pm}$ is the restriction of $\{(t, x, y, z): x=y=0$ and $\pm z>0\}$ from Example (1.4).

(2) $C_{\rho}$ consists of a finite set of components, and $|z|<s_{0} / 100$ on each.

(3) A component of $C^{\prime}$ of $C_{\rho}$ has the following properties:

(a) Where $\rho=\left(x^{2}+y^{2}\right)^{1 / 2}<s_{0}$, the pair of functions $(t, \rho)$ give a proper embedding of $C^{\prime}$ into $\left(t_{0}-s_{0}, t_{0}+s_{0}\right) \times\left(0, s_{0}\right)$.

(b) The restrictions of the functions $z$ and $\phi=\arctan (y, x)$ to $C^{\prime}$ extend from where $t \in\left(t_{0}-s_{0}, t_{0}+s_{0}\right)$ and $\rho \in\left(0, s_{0}\right)$ to where $\rho=0$ as real analytic functions.

(c) The Taylor's expansions of $\phi$ and $z$ off of $\rho=0$ are

$$
\begin{aligned}
\phi & =\phi_{0}(t)-8^{-1} \phi_{0}^{\prime \prime}(t) \cdot \rho^{2}+\mathcal{O}\left(\rho^{4}\right), \\
z & =4^{-1} \phi_{0}^{\prime \prime}(t) \cdot \rho^{2}+\mathcal{O}\left(\rho^{4}\right) .
\end{aligned}
$$

The remainder of this section is occupied with the statement and proof of Proposition 7.2, below; the latter implies Assertions 1 and 2 of Proposition 7.1. The proof of Assertion 3 of Proposition 7.1 is completed in Section 8 and in the Appendix. 
Proposition 7.2 Let $t_{0} \in Z_{R}$. Let $p=p\left(t_{0}\right)$. There is an open neighborhood $U \subset Z_{R}$ of $t_{0}$ with the following significance: Given $\varepsilon$, there exists $s>0$, such that the set of points in $C$ where $t \in U$ and $\rho^{2}+z^{2}<s^{2}$ is a disjoint union $C_{\rho} \cup C_{z+} \cup C_{z-}$ which have the following properties:

- $C_{\rho}$ has at most $p$ components and $|z| / r<\varepsilon$ on each.

- $C_{z \pm}$ is either empty or else the restriction of $\{(t, x, y, z): x=y=0$ and $\pm z>0\}$ to where $t \in U$ and $\rho^{2}+z^{2} \leq s^{2}$.

Aside from giving Assertions 1 and 2 of Proposition 7.1, this last proposition also implies the initial assertion that $p$ and $q_{ \pm}$are constant on $Z_{R}$. Indeed, the constancy of $p$ follows from that of $q_{+}$and $q_{-}$since $p-\left(q_{+}+q_{-}\right)$is the intersection number of $C$ with a linking 2 -sphere around $Z$. The constancy of $q_{ \pm}$follows from the following claim: If $C$ contains a non-empty open set which is identical to an open set in some connected, pseudo-holomorphic subvariety $C^{\prime}$, then $C$ contains the whole of $C^{\prime}$. Because $C$ is assumed closed in $S^{1} \times\left(B^{3}-0\right)$, the latter claim follows from the standard regularity theorems about pseudoholomorphic maps (see, eg [17] or [24]. Alternately, one can invoke Aronszajn's unique continuation theorem in [1].)

The proof of Proposition 7.2 is broken into two parts corresponding to the two points of the proposition.

\section{a) Proof of the first assertion of Proposition 7.2}

Although somewhat lengthy, the first assertion is essentially a corollary of the monotonicity result from Proposition 2.1. In any event, the proof is conveniently divided into four steps.

Step 1 The first observation concerns $\sigma$ from (2.2), but considered as a function of two variables, $t$ and $s$.

Lemma 7.3 Let $t_{0} \in Z_{R}$. Let $p=p\left(t_{0}\right)$ and $q=q\left(t_{0}\right)$. Then there is an open neighborhood $U \subset Z_{R}$ of $t_{0}$ with the following significance: For each $t \in U$, introduce the function $\sigma$ of (2.2) and thus consider the latter as a function, $\sigma(s, t)$ of variables $s>0$ and $t \in U$. Given $\varepsilon>0$, there exists $s_{\varepsilon}>0$ such that for all $s<s_{\varepsilon}$ and $t \in U$,

$$
s^{-3} \sigma(s, t)-(p+2 q) \cdot \frac{2}{3}<\varepsilon .
$$


Proof of Lemma 7.3 Choose the set $U$ to be connected and to have compact closure in $Z_{R}$. This implies that the function $p(\cdot)$ is constant on the closure of $U$. Now, supppose that there exists $\varepsilon>0$, but that no $s_{\varepsilon}>0$ exists which makes (7.2) true for all $t \in U$. Then, there would exist an infinite sequence $\left\{t_{m}\right\}_{m=1, \ldots} \subset U$ such that for each $m$, the inequality in (7.2) is violated using $s=1 / m$. Let $t \in Z$ denote a limit point of the sequence $\left\{t_{m}\right\}$. Since $t \in Z_{R}$ and $p(t)=p$, there exists $s^{\prime}>0$ such that for all $s<s^{\prime}$, the inequality in (7.2) holds with $\varepsilon$ replaced by $\varepsilon / 2$. Fix such $s$. It is an exercise to verify that $\sigma(s, \cdot)$ is a continuous function on $Z$; and this implies that the inequality in (7.2) holds for those $t_{m}$ which are sufficiently close to $t$. This contradicts the initial assumptions and thus proves the lemma.

Step 2 A straightforward modification of the preceding argument proves:

Lemma 7.4 Let $t_{0} \in Z_{R}$ and set $p=p\left(t_{0}\right)$ and $q=q\left(t_{0}\right)$. There is an open neighborhood $U \subset Z_{R}$ of $t_{0}$ with the following significance: For each $t \in U$ and $s>0$, introduce the function

$$
\gamma(s, t)=\int_{c} \chi_{s} \cdot d \phi \wedge d h
$$

Then, given $\varepsilon>0$, there exists $s_{\varepsilon}>0$ such that for all $t \in U$ and $s<s_{\varepsilon}$,

$$
s^{-3} \cdot \gamma(t, s)<\varepsilon \text {. }
$$

(Note that (1.10) and (1.11) imply that $d \phi \wedge d h$ restricts to $C$ as an everywhere non-negative 2 -form.)

Step 3 This step is concerned with the following claim: There exists a neighborhood $U$ of $t_{0}$ and, given $\varepsilon>0$, there exists $s_{\varepsilon}>0$ such that either

$$
|z| / \rho<\varepsilon \quad \text { or } \rho /|z|<\varepsilon
$$

at points of $C$ where $t \in U$ and $\rho^{2}+z^{2} \leq s_{\varepsilon}^{2}$.

To prove this claim, consider an open interval $U \subset Z_{R}$ which contains $t_{0}$, is connected and which has compact closure in $Z_{R}$. Now, suppose that there exists $\varepsilon>0$ and no $s_{\varepsilon}>0$ which makes (7.5) true at points of $C$ where $t \in U$ and $\rho^{2}+z^{2} \leq s_{\varepsilon}^{2}$. There would then be an infinite sequence $\left\{t_{m}\right\} \in U$ with the following properties: There is a point $w_{m}$ on $C$ with $t$ coordinate $t_{m}$, with $r=\left(\rho^{2}+z^{2}\right)^{1 / 2}$ coordinate $r_{m}=1 / m$ and with $r_{m}^{-3} \cdot h\left(w_{m}\right) \geq \varepsilon^{3}$. No generality is lost by assuming that $\left\{t_{m}\right\}$ converges to a point $t_{\infty}$ in the closure of $U$. 
With the preceding understood, for each $m$, rescale so that the ball of radius $4 r_{m}$ centered at $t_{m} \in Z$ becomes the ball of radius 4 . Then, translate along $Z$ so that $t_{m}$ is moved to the origin. The result is a sequence $\left\{C_{m}\right\}$ of pseudoholomophic submanifolds on the set where $t^{2}+\rho^{2}+z^{2}<m / \zeta$ and where $\rho^{2}+z^{2}>1 / 16$. Here, $\zeta \geq 1$ is a fixed constant. Note that $w_{m}$ is sent to a point with $t$ coordinate zero and with $r=1$.

The sequence $\left\{C_{m}\right\}$ has a uniform bound for $\int \omega$ on any fixed ball about the origin. Thus, Proposition 3.3 finds a subsequence which converges geometrically (as described in Definition 3.1) where $\rho^{2}+z^{2}>1 / 16$ to a pseudo-holomorphic subvariety, $C_{\infty}$. Furthermore it follows from Lemma 7.4 that $d \phi=d h=0$ on $C_{\infty}$. This implies that $C_{\infty}$ is a union of subvarieties, where each component has the form $\phi=$ constant and $h=$ constant from Example 1.6. Note in particular, that there must be at least one component of $C_{\infty}$ where $h \geq \varepsilon^{3}$.

To obtain the final contradiction, consider now fixing some $t^{\prime} \in Z$ very near to $t_{\infty}$ and counting the intersections of $C$ with a pseudo-holomorphic disk which obeys the conditions $t=t^{\prime}, f=$ constant $>0$ and $|z| / \rho<1 / 4$. (This is a subdisk of one of the cases in Example 1.6.) Consider, in particular, the case where $f=c$ where $c=\left|t_{\infty}-t^{\prime}\right|$. It then follows from (4.1) (using $t_{\infty}$ in (4.1) instead of $t_{0}$ ) that this intersection number is equal to $p$ when $t^{\prime}$ is sufficiently close to $t_{\infty}$. (Remember that all intersection points count positively to this intersection number.) With the preceding understood, fix $t^{\prime}$ very close $t_{\infty}$ and consider decreasing $c$. For $c$ sufficiently small, it follows from (4.1) (using $t^{\prime}$ for $t_{0}$ in (4.1) this time) that the intersection number is again, equal to $p$. On the other hand, if $t^{\prime}=t_{m}$ and $m$ is large, it follows from the behavior of $C_{m}$ at large $m$ that the intersection number must be less than $p$ when $c$ decreases much past $r_{m}^{2}$.

It follows from the preceding step that there is an open neighborhood $U$ of $t_{0}$ and, given $\varepsilon$, there exists $s_{\varepsilon}>0$ such that where $t \in U$ and $\rho^{2}+z^{2} \leq s_{\varepsilon}^{2}, C$ can be written as $C=C_{\rho} \cup C_{z}$, where $|z| / \rho<\varepsilon$ on $C_{r}$ and $\rho /|z|<\varepsilon$ on $C_{z}$. That is, (7.5) holds.

Step 4 With (7.5) understood, the proof of the first assertion of Proposition 7.2 is complete with the establishment of a bound by $p$ on the number of components of $C_{r}$. To obtain such a bound, agree first to re-introduce the family of pseudo-holomorphic cylinders given by $t=t^{\prime}, f=$ constant $>0$ and $|z| / \rho<1 / 2$ where $t^{\prime}$ now ranges over some small interval $U \subset Z_{R}$ which contains $t_{0}$. If $c>0$, let $\Sigma\left(t^{\prime}, c\right)$ denote the $t=t^{\prime}$ and $f=c$ case.

Now, let $C^{\prime}$ be a component of the intersection of $C_{\rho}$ with some small neighborhood of $t_{0}$ in $S^{1} \times B^{3}$. Note that when $t^{\prime}$ is near to $t_{0}$ and $c$ is small, $\Sigma\left(t^{\prime}, c\right)$ 
has positive intersection number with $C^{\prime}$. (This follows from Proposition 3.2.) Because of (7.5), this intersection number will be independent of both $t^{\prime}$ and $c$ as long as $\left|t^{\prime}-t_{0}\right|$ and $c$ are both small.

On the other hand, the intersection number of $\Sigma\left(t_{0}, c\right)$ with $C_{\rho}$ is, for small $c$, equal to $p$. (This follows by rescaling (4.2).) For such $c$, the intersection number of $\Sigma\left(t^{\prime}, c\right)$ with $C_{\rho}$ must equal $p$ for all $t^{\prime}$ near to $t_{0}$. Furthermore, because of (7.5), this intersection number will also be independent of $t^{\prime}$ and $c$ as long as both $\left|t^{\prime}-t_{0}\right|$ and $c$ are small. Thus, a counting argument shows that there is a small neighborhood of $t_{0}$ in $S^{1} \times B^{3}$ whose intersection with $C_{\rho}$ has at most $p$ components.

\section{b) Proof of the second assertion of Proposition 7.2}

The task here is to verify that $C_{z \pm}$ have the indicated form. To begin, consider a component $C^{\prime}$ of $C_{z}$. It follows from (7.5) that one can assume, without loss of generality that $z>0$ on this component. Concerning $(t, u)$, consider:

Lemma 7.5 The functions $t$ and $u=(-f)^{1 / 2}$ restrict to a neighborhood of all but a set of at most countably many points in $C^{\prime}$ as local coordinates. The countable set, $\Lambda$, here consists of the singular points of $C^{\prime}$ and the critical points of $d t$, (or, equivalently, $d u$ ). Note that this set $\Lambda$ has no accumulation points in $C^{\prime}$.

Proof of Lemma 7.5 By assumption, $C^{\prime}$ has at most a countable number of singular points. With the preceding understood, let $o \in C^{\prime}$ be a smooth point. Then, by assumption, there is a pseudo-holomorphic embedding $f$ of the standard disk in $\mathbb{C}$ into $S^{1} \times\left(B^{3}-\{0\}\right)$ which sends 0 to $o$ and the disk to a neighborhood of $o$ in $C^{\prime}$. Furthermore, it is an exercise to show that there are coordinates $\left(\sigma^{1}, \sigma^{2}\right)$ on the disk with respect to which the pull-backs of the functions $t$ and $u$ obey the equations

$$
t_{1} \kappa=u_{2} \quad \text { and } \quad t_{2} \kappa=-u_{1} .
$$

Here, $\kappa=\left(\rho^{2}+4 z^{2}\right)^{1 / 2} / 2 u=1+\mathcal{O}\left(r^{2} / z^{2}\right)$. (In (7.6), the subscripts " 1 " and "2" denote the partial derivatives with respect to $\sigma_{1}$ and $\sigma_{2}$.) Equation (7.6) implies, first of all, that the critical points of $t$ and $u$ agree on $C^{\prime}$.

Now, suppose that $o$ were also an accumulation point of critical points of $d t$. The argument below deduces a contradiction from this supposition. To begin the argument, note first that (7.6) implies a homogeneous Laplace equation for either $t$ or $u$ on the disk. The equation for $t$ reads: $\left(\kappa t_{1}\right)_{1}+\left(\kappa t_{2}\right)_{2}=0$. This 
equation implies, via Aronszajn's unique continuation theorem [1], that $t-t(0)$ does not vanish to infinite order at 0 .

On the other hand, if 0 were an accumulation point of the critical set of $t$, then Taylor's theorem with remainder and the Laplace equation would force $t-t(0)$ to vanish to infinite order at 0 . The argument for this last assertion is as follows: If $t$ has a non-zero Taylor's coefficient, then it has one of smallest order, say $n$. With $n$ understood, use Taylor's theorem to write $t=t(0)+$ $\sum_{0 \leq k \leq n} a_{k} \sigma_{1}^{k} \sigma_{2}^{n-k}+\mathcal{O}\left(|\sigma|^{n+1}\right)$. The definition of $n$ requires some $a_{k} \neq 0$. If $d t$ is to vanish arbitrarily close to 0 , then there must exist $\left(\sigma_{1}, \sigma_{2}\right) \neq 0$, with the property that $\sum_{0 \leq k \leq n} a_{k} k \sigma_{1}^{k-1} \sigma_{2}^{n-k}=0$ and $\sum_{0 \leq k \leq n} a_{k}(n-k) \sigma_{1}^{k} \sigma_{2}^{n-k-1}=0$. By rotating the coordinate system if necessary, one can assume, with out loss of generality, that this occurs where $\sigma_{2}=0$. Thus, the vanishing of $d t$ at points arbitrarily close to 0 implies that $a_{n-1}=a_{n}=0$. Meanwhile, the aforementioned Laplace equation for $t$ requires that the sum $\sum_{0 \leq k \leq n} a_{k}\left(k(k-1) \sigma_{1}^{k-2} \sigma_{2}^{n-k}+(n-k)(n-k-1) \sigma_{1}^{k} \sigma_{2}^{n-k-2}\right)$ vanish at all $\sigma$. This last fact implies, via an induction on $n-k$, that $a_{k}=0$ for all $k$, thus contradicting the defining property of $n$.

With the preceding lemma understood, remark that a neighborhood of any point in $C^{\prime}-\Lambda$ where $\rho^{2}+z^{2}$ is small is given as the set of points $(t, z, x, y)$ where

- $\quad x=x(t, u), \quad y=y(t, u)$

- $z^{2}=u^{2}+2^{-1} \cdot\left(x^{2}+y^{2}\right)$

- $u \in\left(0, s_{1}\right)$ and $t \in U$.

Here, $s_{1}$ is positive and much less than $s_{0}$. In particular, given $\varepsilon>0$, one can choose $s_{1}$ so that

$$
(|x|+|y|) / u<\varepsilon .
$$

Here, $x$ and $y$ are implicitly restricted to a neighborhood of the given point in $C^{\prime}$ where they are considered as functions of the variables $t$ and $u$.

Now, let $\eta=x+i \cdot y$ and introduce the complex coordinate $w=t+i \cdot u$ and the associated $\partial=2^{-1} \cdot\left(\partial_{t}-i \cdot \partial_{u}\right)$. Then, $\eta$ obeys an equation of the form

$$
\partial \eta+i \cdot \eta / 4 z=a \cdot \nabla \eta+b \cdot \nabla \bar{\eta},
$$

where $a$ and $b$ are functions of $u$ and $\eta$ which obey $|a|+|b| \leq \zeta \cdot|\eta|^{2} / u^{2}$. Note that (7.9) is augmented with the knowledge that $|\eta| / u$ is uniformly bounded by $\varepsilon$. Furthermore,

$$
\lim _{u \rightarrow 0}|\eta| / u=0 .
$$


Now, it is important to realize that the set $\Lambda \subset C^{\prime}$ need not be empty, and may, as far as has been established as yet, have infinitely many members. In particular, for the sake of argument, one can assume that $\Lambda$ has an infinite number of elements, for othewise, one could decrease $s_{0}$ and thus avoid them entirely in the subsequent discussion.

In any event, the complex function $\eta$ obviously extends (by definition) as a continuous function on $C^{\prime}$, but not so as a global function of the complex coordinate $w$. As a function of the latter, $\eta$ is multivalued, where the different values correspond to different sheets of $C^{\prime}$ over the $w$ plane. The points of $\Lambda$ then correspond to places where the sheets coincide. (In any event, there are at most $q_{+}$sheets because each sheet has intersection number 1 with a $t=$ constant, $f=$ constant $<0$ psuedo-holomorphic submanifold.)

The structure of $C^{\prime}$ as viewed using the complex coordinate $w$ can be described slightly differently as follows: The assignment of the complex number $t+i u$ to a point in $C^{\prime}$ defines a continuous map, $\pi$, from $C^{\prime}$ to the $(t, u)$ plane. In a neighborhood of $w \notin \pi(\Lambda)$, the sheets of $C^{\prime}$ are parametrized by a set $\left\{\eta_{1}, \ldots, \eta_{q^{\prime}}\right\}$ of complex valued functions with distinct values at each point. Here, $q^{\prime} \leq q_{+}$is fixed. Each $\eta=\eta_{j}$ obeys (7.9) and (7.10). Meanwhile, the points in $\pi(\Lambda)$ are characterized by the fact that the values of some pair from $\left\{\eta_{1}, \ldots, \eta_{q^{\prime}}\right\}$ coincide. Furthermore, as $w$ traverses a circle around some point in $\pi(\Lambda)$, the set $\left\{\eta_{1}, \ldots, \eta_{q^{\prime}}\right\}$ can be permuted by some non-trivial monodromy.

With the preceding understood, consider now the complex valued function

$$
\theta=\prod_{1 \leq j \leq q^{\prime}} \eta_{j}
$$

Note that $\theta$ is a globally defined function on a neighborhood of 0 in the upper half of the $w$ plane which obeys

$$
\lim _{u \rightarrow 0} \theta / u^{q^{\prime}}=0 .
$$

Furthermore, by virtue of (7.9), this complex valued function obeys an equation of the form

$$
\partial \theta+i \cdot \theta \cdot q^{\prime} \cdot(1+m) / 4 u=0,
$$

where $m$ is a smooth complex valued function away from $\pi(\Lambda)$ about which more will be said below.

Here is the derivation of (7.13): First, observe that both the Euclidean metric and $\omega$ are invariant under the group of rotations in the $(\mathrm{x}, \mathrm{y})$ plane. This implies that if $\eta=\eta_{j}$ is a solution to (7.9), then so is $\lambda \cdot \eta_{j}$ where $\lambda$ is any unit length complex number. This fact implies that the functions $a$ and $b$ in (7.9) must have the form 
- $\quad a=\alpha \cdot\left|\eta_{j}\right|^{2} / u^{2}$

- $\quad b=\beta \cdot \eta_{j}^{2} / u^{2}$.

Here, $\alpha$ and $\beta$ are functions of $u$ and $\eta_{j}$ which obey $|\alpha|+|\beta| \leq \zeta$. With (7.14) understood, then (7.9) for $\eta=\eta_{j}$ can be written as $\partial \eta_{j}+i \cdot \eta_{j} \cdot\left(1+m_{j}\right) / 4 u=0$, where

$$
m_{j}=(u / z-1)-4 \cdot\left(\alpha \cdot \bar{\eta}_{j} \cdot \nabla \eta_{j}+\beta \cdot \eta_{j} \cdot \nabla \bar{\eta}_{j}\right) / u .
$$

With $m_{j}$ understood, then (7.13) for $\theta=\prod_{j} \eta_{j}$ follows immediately with $m=$ $\sum_{j} m_{j}$.

The subsequent manipulations of (7.13) require an estimate for the size of $m$. Here, remark that $m$ is smooth away from the points in $\pi(\Lambda)$. The following lemma describes $|m|$ in more detail.

Lemma 7.6 Although $|m|$ may be unbounded near the point of $\Lambda$, this function is none the less locally square integrable. Moreover, there exists $c \geq(0,1)$ and, given $\varepsilon>0$, there exists $s_{\varepsilon}>0$ such that the following is true: Suppose that $s, r \in\left(0, s_{\varepsilon}\right)$. Let $A$ denote the intersection of the $u \geq 0$ portion of the $(t, u)$ plane with an annulus with center where $|t|<c$ and $u=s$, and whose inner and outer radii are $r / 2$ and $r$, respectively. Then:

- If $r \leq s / 4$, then $\int_{A}|m|^{2} d t d u \leq \varepsilon^{2}(r / s)^{c} s^{2}$.

- If $r \geq s / 4$, then $\int_{A}|m|^{2} d t d u \leq \varepsilon^{2} r^{2}$.

This lemma is proved below. Accept it for now.

With (7.13) understood, then the second assertion of Proposition 7.2 follows with a proof that the only solution to (7.12) with $\lim _{u \rightarrow 0} \theta=0$ is the function $\theta \equiv 0$. (Remember that $C^{\prime}$ is assumed to be connected.)

Progress on this task requires

Lemma 7.7 Given $c>0$, there exists a constant $\zeta>0$ with the following significance: Let $m$ be a square integrable, complex valued function on the $(t, u)$ upper half plane which obeys the conclusions of Lemma 7.6 for some $\varepsilon>0$ and for the given constant $c$. Suppose in addition that there exists $R \in(0, c)$ such that $m=0$ where $\left(t^{2}+u^{2}\right)^{1 / 2} \geq R$. Then there exists a real valued function, $\kappa$, on the $(t, u)$ upper half plane which, where $t^{2}+u^{2} \leq R / 2$, obeys

- $\quad \partial \kappa+i \cdot \kappa \cdot(1+m) / 4 u=0$

- $\quad \kappa>0$ 
- $\quad e^{-4 \varepsilon} u^{q^{\prime} / 2+\zeta \varepsilon} \leq \kappa \leq u^{q^{\prime} / 2-\zeta \varepsilon}$.

This lemma is also proved momentarily. Accept it for now to complete the analysis of (7.13).

In particular, take an open set $U^{\prime} \subset U$ with compact closure in $U$, and take some very small $\varepsilon>0$ in Lemma 7.6 to obtain $s_{\varepsilon}$. Then, modify $m$ from (7.13) with a cut off function so that the result (still to be called $m$ ) is unchanged when $(t, u) \in U^{\prime} \times\left(0, s_{\varepsilon} / 2\right)$ but vanishes when $(t, u)$ is sufficiently close to the frontier of $U \times\left(0, s_{\varepsilon}\right)$. With this $m$ understood, use Lemma 7.7 to produce $\kappa$. Then, note that $\theta^{\prime}=\kappa \cdot \theta$ obeys the equation $\partial \theta^{\prime}=0$ on $U^{\prime} \times\left(0, s_{\varepsilon} / 2\right)$ and extends as 0 on $U^{\prime} \times\{0\}$. The latter implies that $\theta^{\prime} \equiv 0$ as this is the only anti-holomorphic function which vanishes on a line segment. Thus, $\theta \equiv 0$ as claimed.

The proof of the Proposition 7.2 is thus completed with the proofs of Lemmas 7.6 and 7.7. These will be taken in reverse order.

Proof of Lemma 7.7 Write $\kappa=u^{q^{\prime} / 2} \cdot e^{\tau}$ and then $\tau$ must obey the equation

$$
\partial \tau+i \cdot m / 4 u=0 .
$$

Here is one solution to (7.16):

$$
\tau(w)=-(4 \pi)^{-1} \int_{i m\left(w^{\prime}\right)>0} m\left(w^{\prime}\right)\left(\left(\bar{w}-\bar{w}^{\prime}\right)^{-1}-\left(\bar{w}-w^{\prime}\right)^{-1}\right) / i m(w) d \bar{w}^{\prime} \wedge d w^{\prime}
$$

To see that this integral is well defined, note that the integral above can be rewritten as

$$
\tau(w)=-i(2 \pi)^{-1} \int_{y \geq 0} m\left(w^{\prime}\right) /\left(\left(\bar{w}-\bar{w}^{\prime}\right)\left(\bar{w}-w^{\prime}\right)\right) d \bar{w}^{\prime} \wedge d w^{\prime} .
$$

To estimate the norm of $\tau$, consider breaking the domain of integration into two parts. The first part has $\left|w-w^{\prime}\right| \leq \operatorname{im}(w)=2 u$; and the second has $\left|w-w^{\prime}\right| \geq 2 u$. Because $\left|w-\bar{w}^{\prime}\right| \geq u$ when $w$ and $w^{\prime}$ lie in the upper half plane, the contribution from the first region has absolute value no larger than

$$
(2 \pi)^{-1} u^{-1} \int_{\left|w-w^{\prime}\right| \leq 2 u}|m|\left|w-w^{\prime}\right|^{-1} i d \bar{w}^{\prime} \wedge d w^{\prime} .
$$

The claim here is that (7.19) is no greater than $\zeta \varepsilon$, where $\zeta$ depends only on the constant $c$, but not on $\varepsilon, R$ or on other properties of $m$. Here is a proof of this claim: Break the integration region into concentric annuli indexed by $n \in\{0,1, \ldots\}$, where the $n$ 'th annulus is characterized by the requirement that $2^{-n} u \leq\left|w-w^{\prime}\right| \leq 2^{-n+1} u$. The contribution to (7.19) from the $n$ 'th annulus 
is no greater than $\pi^{-1} 2^{n} u^{-2}$ times the $L^{1}$ norm of $|m|$ over the $n$ 'th annulus. This number is no greater than $\sqrt{2} u^{-1}$ times the $L^{2}$ norm of $|m|$ over this same annulus. And, according to Lemma 7.6, the $L^{2}$ norm in question is no greater than $\varepsilon\left(2^{-n}\right)^{c / 2} u$. Thus, the n'th annulus contributes no more that $\sqrt{2} \varepsilon 2^{-n c / 2}$ to the integral in (7.19). With this last point understood, it follows that (7.19) is no greater than $\sqrt{2} \varepsilon \sum_{n \geq 0} 2^{-n c / 2}$. As this last sum converges, the asserted uniform bound of (7.19) by $\zeta \varepsilon$ is obtained.

Meanwhile, the absolute value of the contribution to (7.18) from the second region is no larger than

$$
(2 \pi)^{-1} \cdot \int_{\left|w-w^{\prime}\right| \leq 2 u}\left|m\left(w^{\prime}\right)\right| /\left|w^{\prime}-w\right|^{2} i \cdot d \bar{w}^{\prime} \wedge d w^{\prime} .
$$

The latter integral can be bounded by breaking the integration region into $u \geq 0$ half annuli, where these half annuli are indexed by $n \in\{1, \ldots, N\}$, here $N \leq 1+4 \ln (R / u)$. In this case, the $n$ 'th half annulus is characterized by $2^{n} u \leq\left|w-w^{\prime}\right| \leq 2^{n+1} u$. The contribution to (7.20) from the $n$ 'th half annulus is no greater than $(2 \pi)^{-1} 2^{-2 n} u^{-2}$ times the $L^{1}$ norm of $|m|$ over said half annulus. Meanwhile, this $L^{1}$ is no greater than $(2 \pi)^{-1} 2^{-2 n} u^{-2}$ times the $L^{2}$ norm of $|m|$ over the half annulus in question. Apply the second point in Lemma 7.6 to bound this last $L^{2}$ norm by $\varepsilon 2^{n+1} u$, and thus bound the contribution from the n'th half annulus by a constant $4 \varepsilon$. With the preceding understood, it follows that (7.20) is no greater than $4 \varepsilon N \leq 16 e|\ln u|$. Thus,

$$
|\tau| \leq \zeta \varepsilon \cdot(1+|\ln u|)
$$

where $\zeta$ depends only on the constant $c$ in the statement of the lemma.

This section ends with the proof of Lemma 7.6.

Proof of Lemma 7.6 The proof of Lemma 7.6 is broken into six steps.

Step 1 It proves useful for a subsequent application in Section 9, to establish a version of Lemma 7.6 under slightly weaker assumptions then those given. Thus, the proof starts with a digression to review the background for these assumptions.

To start the digression, suppose that $s_{0} \in(0,1 / 4)$ has been fixed, together with a constant $\alpha \geq 0$. Let $W$ denote the domain in the $(t, u)$ plane where

$$
\text { - } t^{2}+u^{2}<s_{0}
$$

- $u>\alpha|t|$. 
Let $W^{\prime} \subset S^{1} \times\left(B^{3}-\{0\}\right)$ denote the domain where $z>0, \rho / z<1 / 4$ and where (7.22) holds using $u=(-f)^{1 / 2}$. (The function $u$ is well defined where $0 \leq \rho / z<1 / 4$.)

Now, suppose that $C^{\prime} \subset W^{\prime}$ is a finite energy, pseudo-holomorphic subvariety for which the following regularity assumptions hold: Given $\varepsilon>0$, there exists $s_{\varepsilon}>0$ such that when $s<s_{\varepsilon}$, then

- $\rho / u<\varepsilon$

- $\int_{C^{\prime} \cap\{u<s\}} d \phi \wedge d h \leq \varepsilon s^{3}$.

Note that Lemma 7.4 and (7.7) guarantee (7.23) for the subvariety in Lemma 7.6 .

With $C^{\prime}$ as described above, the association of $w=t+i u$ to its points defines a continuous map, $\pi$, from $C^{\prime}$ to the $u>0$ half of the $(t, u)$ plane. As with the example from Lemma 7.6, the function $w$ (via the map $\pi$ ) defines a complex coordinate near all but a set, $\Lambda$, of at most countably many points of $C^{\prime}$. This set $\Lambda$ contains the singular points of $C^{\prime}$ and the critical points of $u$ (or $t$ ).

The push forward by this map $\pi$ of the function $\eta \equiv(x+i y)$ defines an unordered set $\left\{\eta_{j}\right\}$ of some number, $q^{\prime}$, of locally defined functions on the domain $W$ in (7.22). (Note that $q^{\prime}$ is a constant, as it measures the intersection number of $C^{\prime}$ with certain members of the $\left(t=c, f=c^{\prime}\right)$ case of Example 1.6.) These $\left\{\eta_{j}\right\}$ can be ordered as smooth functions near any point which is not in $\pi(\Lambda)$. Note that each $\eta_{j}$ satisfies (7.9) at points not in $\pi(\Lambda)$. The coorresponding $m_{j}$ is still given by (7.15).

With the preceding understood, set $m=\sum_{j} m_{j}$. Then, the assertions of Lemma 7.6 make sense in the present context if $A$ is suitably interpreted. In particular, consider the following lemma:

Lemma 7.8 Let $W, W^{\prime}$ and $C^{\prime}$ be as described above. Let $m=\sum_{j} m_{j}$. Then $|m|$ is locally square integrable. Moreover, there exists $c \subset(0,1)$ and, given $\varepsilon>0$, there exists $s_{\varepsilon}>0$ such that the following is true: Suppose that $s, r \in\left(0, s_{\varepsilon}\right)$. Let $A$ denote the intersection of $W$ with an annulus whose center has $|t|<\min (c, s /(2 \alpha))$ and $u=s$, and whose inner and outer radii are $r / 2$ and $r$, respectively. Then:

- If $r \leq s / 4$, then $\int_{A}|m|^{2} d t d u \leq \varepsilon^{2}(r / s)^{c} s^{2}$.

- If $r \geq s / 4$, then $\int_{A}|m|^{2} d t d u \leq \varepsilon^{2} r^{2}$. 
This lemma is reduced in the next step to a related lemma.

Step 2 It follows from (7.15) that

$$
\left.|m|^{2}\right|_{A} \leq \zeta \cdot\left[\sup _{w \in A}\left(\sum_{j}\left|\eta_{j}\right| / u\right)\right]^{2} \sum_{j}\left|d \eta_{j}\right|^{2} .
$$

Then, (7.22) implies that given $\delta>0$, there exists $s_{\delta}^{\prime}$ such that when $r, s \in$ $\left(0, s_{\delta}^{\prime}\right)$ then the left-hand side of (7.24) is no greater than $\zeta \delta^{2} \sum_{j}\left|d \eta_{j}\right|^{2}$. Thus, it is enough to bound the integral of $\sum_{j}\left|d \eta_{j}\right|^{2}$ over the region $A$.

With the preceding understood, introduce the function $\lambda \equiv \sqrt{z} \eta$ and the corresponding set $\left\{\lambda_{j}=\sqrt{z} \eta_{j}\right\}$. Since $\rho / u<<1$ on $C^{\prime}$ in the region of interest, one has

$$
\left|d \eta_{j}-z^{-\frac{1}{2}} d \lambda_{j}\right|^{2} \leq \zeta\left|d \eta_{j}\right| / u
$$

Hence, it is more than sufficient for the proof of Lemma 7.8 to establish the following lemma:

Lemma 7.9 Let $W, W^{\prime}$ and $C \subset W^{\prime}$ be as described above. The function $\sum_{j}\left|d \lambda_{j}\right|^{2}$ on $W$ is locally integrable. Moreover, there exists $c>0$, and, given $\varepsilon$, there exists $s_{\varepsilon}>0$ which are such that when $r, s \in\left(0, s_{\varepsilon}\right)$, then:

- Suppose that $r \leq s / 4$. Let $D \subset W$ denote the disk with center where $|t| \leq \min (c, u /(2 \alpha))$ and $u=s$, and with radius $r$. Then, $\int_{D} \sum_{j}\left|d \lambda_{j}\right|^{2} \leq$ $\varepsilon^{2}(r / s)^{c} s^{3}$.

- In general, $\int_{\substack{\alpha|t| \leq u \leq r \\|t| \leq c}} \sum_{j}\left|d \lambda_{j}\right|^{2} \leq \varepsilon^{2} r^{3}$.

Proof of Lemma 7.8 given Lemma 7.9 The local integrability of $\sum_{j}\left|d \eta_{j}\right|^{2}$ follows from that of $\sum_{j}\left|d \lambda_{j}\right|^{2}$, and the first point follows directly from the corresponding point of Lemma 7.9. The second point of Lemma 7.8 follows from that of Lemma 7.9 by decomposing the region $A$ of Lemma 7.8 into subregions indexed by $n \in\{1,2, \ldots\}$, where the $n$ 'th subregion is defined by the constraint $2^{-n r} \leq u \leq 2^{-n+1} r$. Then, the integral of $|z|^{-1} \sum_{j}\left|d \lambda_{j}\right|^{2}$ over the $n$ 'th subregion is, according to the second point in Lemma 7.9, no greater than the following: $\varepsilon^{2}\left(2^{-n} r\right)^{-1}\left(2^{-n+1} r\right)^{3}=8 \varepsilon^{2} 2^{-2 n} r^{2}$. Summing the latter contributions over $n$ bounds the desired integral by $8 / 3 \varepsilon^{2} r^{2}$. Thus, the second point of Lemma 7.8 follows if $s_{\varepsilon}$ in the statement of said lemma is taken to equal $s_{\varepsilon^{\prime}}$ from Lemma 7.9 where $\varepsilon^{\prime}=\varepsilon(3 / 8)^{1 / 2}$.

Step 3 This step and the subsequent three steps contain the proof of Lemma 7.9 . 
Proof of Lemma 7.9 Consider first the assertion that $\sum_{j}\left|d \lambda_{j}\right|^{2}$ is locally integrable. For this purpose, note that the behavior of $\lambda$ near points in $\Lambda$ can be discerned from the known local structure of a pseudo-holomorphic map as described, for example, in [17] or [24]. (See also [14].) In particular, the claim here is that if $w_{0} \in \Lambda$, then there exists a constant $\zeta \geq 1$ such that

$$
\sum_{j}\left|d \lambda_{j}\right|^{2} \leq \zeta\left|w-w_{0}\right|^{-2+2 / \zeta}
$$

at points $w$ near, but not equal to $w_{0}$.

To prove (7.26), suppose that $o \in \Lambda$. Then, the analysis in [24] or [17] shows that $C^{\prime}-o$ near $o$ is a disjoint union of smooth submanifolds. Moreover, the closure of any such submanifold is the image of the standard disk in $\mathbb{C}$ via an almost everywhere $1-1$, pseudo-holomorphic map $f$ into $S^{1} \times\left(B^{3}-\{0\}\right)$. This map $f$ can be assumed to send the origin in $\mathbb{C}$ to $o$.

In addition, the coordinate $v$ on $\mathbb{C}$ can be chosen so that the pull-back via $f$ of the function $\mathrm{w}$ obeys an equation of the form

$$
\bar{\partial} w+\gamma_{1} \partial w+\gamma_{2} \bar{\partial} \bar{w}=0
$$

where $\gamma_{1,2}$ are smooth functions which obey $\gamma_{1}(0)=0$ and $\left|\gamma_{2}\right| \leq \zeta|\eta|^{2} / u^{2}$. Here, $\partial$ is the complex s derivative on $\mathbb{C}$. (This last equation follows readily from (7.6).) Now, $w$ is a smooth function of $v$ and so has a Taylor's series. By virtue of (7.27), the latter must be such that

$$
w+\gamma_{2}(0) \bar{w}=a_{0}+a_{1} v^{n}+\mathcal{O}\left(|v|^{n+1}\right),
$$

where $n$ is a positive integer and where $a_{0}$ and $a_{1}$ are constants, with neither non-zero when $\left|\gamma_{2}\right|<1$.

Now, to prove (7.26), first use (7.28) to express $v$ as a function of $w$. Then, as $\lambda$ pulls back via $f$ as a smooth function, the desired result follows using the chain rule.

Step 4 To consider the two points of the lemma, remark that by virtue of (7.9) and (7.15), each $\lambda \in\left\{\lambda_{j}\right\}$ obeys an equation of the form

$$
\partial \lambda+\mathcal{P}=0,
$$

at any point not in $\pi(\Lambda)$. Here, $|\mathcal{P}| \leq \zeta(|\lambda| / u+|d \lambda|)|\eta| / u$ and now $\partial$ is the complex $w$ derivative. Then, using (7.23), one can assume the following: Given $\delta$, there exists $s_{\delta}^{\prime}>0$ such that when $u<s_{\delta}^{\prime}$, then

$$
|\mathcal{P}| \leq \delta\left(u^{\frac{1}{2}}+|d \lambda|\right) .
$$


This last equation implies that where $u \leq s_{\delta}^{\prime}$, then

$$
\left(\zeta^{-1}|d \lambda|^{2}-\zeta \delta^{2} u\right) d t \wedge d u \leq-i d \lambda \wedge d \bar{\lambda}
$$

where $\zeta \geq 1$ is a fixed constant.

Equation (7.31) is the basic inequality for the subsequent arguments.

Step 5 This step considers the second integral inequality in Lemma 7.9. For this purpose, note that $\left\{\lambda_{j}\right\}$ can also be written as the pushfoward via $\pi$ from $C^{\prime}$ of $|h|^{1 / 2} e^{i \phi}$. Thus, $i d \lambda \wedge d \bar{\lambda}$ is equal to $d h \wedge d \phi$. With this understood, (7.29) implies that

$$
\int_{C^{\prime} \cap\{u \leq r\}} \sum_{j}\left|d \lambda_{j}\right|^{2} \leq \zeta\left(\delta^{2} r^{3}+\int_{C^{\prime} \cap\{u \leq r\}} d \phi \wedge d h\right) .
$$

It then follows from (7.23) and (7.32) that given $\varepsilon>0$, there exists $s_{\varepsilon}>0$ for which the final point of Lemma 7.9 holds when $r<s_{\varepsilon}$.

Step 6 This step considers the first integral inequality in Lemma 7.9. The domain $D$ here is the full disk of radius $r$. With this understood, introduce $f(r)=\int_{D} \sum_{j}\left|d \lambda_{j}\right|^{2}$. Let $C^{\prime}(r) \subset C^{\prime}$ denote the $\pi$-inverse image of $D$. Then, (7.29) implies that

$$
f(r) \leq \zeta \delta^{2} r^{2} s-i \int_{C^{\prime}(r)} d \lambda \wedge d \bar{\lambda}
$$

When $r$ is such that $\partial C^{\prime}(r)$ is disjoint from $\Lambda$, then the latter is the union of a set, $\{\Gamma\}$, of smoothly embedded circles. With this last observation, use Stoke's theorem to rewrite the integral on the right-hand side above as

$$
-i \int_{C^{\prime}(r)} d \lambda \wedge d \bar{\lambda}=-i \sum_{\Gamma \subset \partial C^{\prime}(r)} \int_{\Gamma}\left(\lambda-\lambda_{\Gamma}\right) d \bar{\lambda}
$$

Here, the sum in question is over the set $\{\Gamma\}$ of components of $\partial C^{\prime}(r)$, and $\lambda_{\Gamma}$ can be any constant, although a convenient choice is made below. Note that there are no anomalous boundary terms in (7.34) arising from the points in $\Lambda$. (The absence of such terms can be verified by pulling the integral on the righthand side of (7.34) back to the smooth model for $C^{\prime}$ via its pseudo-holomorphic map into $S^{1} \times\left(B^{3}-\{0\}\right)$. Up on the smooth model, the function $\lambda$ is smooth while the inverse image of the points in $L \cap D$ is also a finite set.)

With regard to (7.34), note that the number of components of $\partial C^{\prime}(r)$ is no greater than the number, $q^{\prime}$, of elements in the set $\left\{\eta_{j}\right\}$. In addition, the projection $\pi$ maps each component $\Gamma$ of $\partial C^{\prime}(r)$ as a covering map onto $\partial D$ with some covering degree $q_{\Gamma} \leq q^{\prime}$. 
With the preceding understood, here is how to define $\lambda_{\Gamma}$ : Let $t \in[0,2 \pi)$ denote a standard angle parameter on $\partial D(r)$. Then, set

$$
\lambda_{\Gamma} \equiv\left(2 \pi r^{\prime} q_{\Gamma}\right)^{-1} \int_{\Gamma} \lambda \pi^{*} d \tau
$$

It then follows from the fundamental theorem of calculus that the right-hand side of (7.34) has absolute value no greater than

$$
\zeta \cdot \sum_{\Gamma \subset \partial C^{\prime}(r)} r \int_{\Gamma}\left|\lambda_{\tau}\right|^{2} \pi^{*} d \tau \leq \zeta r \sum_{j} \int_{\partial C^{\prime}(r)}\left|d \lambda_{j}\right|^{2} d \tau .
$$

Here, $z \geq 1$ is independent of $r$ and of the center which defines $D$. (The subscript " $\tau$ " in (7.36) indicates the $\tau$-partial derivative.)

Together, (7.33) and (7.36) imply the differential inequality

$$
f \leq \zeta\left(\delta^{2} r^{2} s+r f^{\prime}\right)
$$

This last equation can be integrated from any value of $r<s / 4$ to $r=s / 4$ and the result is the inequality

$$
f(r) \leq 4^{1 / \zeta}(r / s)^{1 / \zeta}\left(f(s / 4)+\zeta \delta^{2} s^{3}\right)
$$

To end the story, note that it has already been established that there exists, given $\delta>0$, a number $s_{\delta}^{\prime}>0$ such that $f(s / 4) \leq \delta^{2} s^{3}$ when $s<s_{d}^{\prime}$. Thus, the right side of (7.38) is no greater than $\zeta(r / s)^{1 / \zeta} d^{2} s^{3}$ when $s<s_{\delta}^{\prime}$. With this last fact given, take $c$ in Lemma 7.9 to equal $1 / \zeta$ and take $s_{\varepsilon}$ to equal $s_{\delta}^{\prime}$ for $\delta=\varepsilon / \sqrt{\zeta}$.

\section{A proof that $C_{\rho}$ is smooth}

The purpose of this section is to prove Assertions 3(b) and 3(c) of Proposition 7.1 under the assumption of Assertion 3(a). Assertion 3(a) is also proved here in the case where $p=1$, but the proof of Assertion 3(a) for $p>1$ is relegated to the Appendix. (The latter proof is quite lengthy.)

The remainder of this section is divided into two subsections. Subsection 8.a establishes some general continuity properties of $C_{\rho}$ near $\rho=0$ when parametrized as a multi-valued map of the $(t, \rho)$ coordinates. Subsection 8.b uses the results from subsection 8.a to prove the $p=1$ version of Assertion 3(a), and also Assertions 3(b) and 3(c) of Proposition 7.1 under the assumption that Assertion 3(a) holds.

Note that because of the second assertion of Proposition 7.2, no generality is lost by assuming that $C=C_{\rho}$ near the given point $t_{0} \in Z_{R}$. This assumption will be made implicitly below. 


\section{8.a Holder continuity}

This subsection establishes some general continuity results for $C$ near a regular point $t_{0} \in Z$. These are used subsequently here, in Section 9 and in the Appendix. The discussion in this subsection is broken into five steps.

Step 1 First, agree to simplify the notation by taking linear coordinates on $Z$ near the regular point $t_{0}$ so that $t_{0}=0$.

Observe that the first assertion of Proposition 7.2 gives an open interval $(-\delta, \delta)$ of 0 in $Z_{R}$, and, with $\varepsilon>0$ chosen, a number $s_{\varepsilon}>0$ so that $|z| / \rho<\varepsilon$ where $C$ intersects the set where $t \in(-\delta, \delta)$ and where $\rho^{2}+z^{2}<s_{\varepsilon}$

With the preceding understood, fix $\varepsilon>0$ but with $\varepsilon<<1 / 100$ and let $C^{\prime}$ be a component of the intersection of $C$ with the set where $t \in(-\delta, \delta)$ and $\rho^{2}+z^{2}<s_{\varepsilon}$. On $C^{\prime}$, the pair of functions $\left(t, u \equiv(2 f)^{1 / 2}=\left(\rho^{2}-2 z^{2}\right)^{1 / 2}\right)$ restrict as coordinates to some disk neighborhood of all but a countable set, $\Lambda$, of points of $C^{\prime}$. This set $\Lambda$ consists of the singular points of $C^{\prime}$ and the critical points of the restriction of $t$. (The critical points of $t$ and $u$ are identical.) Note that $\Lambda$ has no accumulation points on $C^{\prime}$. The fact that $\Lambda$ is countable with no accumulation points is proved by an argument which copies, almost verbatim, the proof of Lemma 7.5. In fact, the only change in the argument is that $\kappa$ in (7.6) should be taken to be $\kappa=-\left(\rho^{2}+z^{2}\right)^{1 / 2} / u=1+O\left(|z|^{2} / \rho^{2}\right)$.

Note that the association of $(t, u)$ to the points in $C^{\prime}$ defines a continuous map, $\pi$, from $C^{\prime}$ to the $u>0$ half of the $(t, u)$ plane. One then has:

Lemma 8.1 There exists $s_{0}>0$ and $\delta>0$ and a positive integer $p^{\prime} \leq p$ with the following significance: Suppose $(t, u) \in(-\delta, \delta) \times\left(0, s_{0}\right)$ and $(t, u) \notin \pi(\Lambda)$. Then $\pi^{-1}(t, u)$ consists of precisely $p^{\prime}$ points.

Proof of Lemma 8.1 The number of points in $\pi^{-1}\left(t_{0}, u_{0}\right)$ counts the intersection number of $C^{\prime}$ with the $(t, f)=\left(t_{0}, u_{0}^{2}\right)$ version of Example 1.6. This intersection number is no greater than $p$ by the definition of $p$ in Section 4 . The fact that this intersection number is constant on $p^{\prime}$ follows from the invariance of intersection number under perturbations.

The functions $(t, u)$ are preferred coordinates on $C^{\prime}$ for the following reason: The fact that $C^{\prime}$ is pseudo-holomorphic implies (and is implied by) a reasonably simple differential equation for $\phi$ and $h=\rho^{2} z$ :

- $h_{u}=\rho^{2} \cdot u \cdot \phi_{t}$

- $h_{t}=-g^{2} \cdot \rho^{2} \cdot u^{-1} \cdot \phi_{u}$. 
Here, the subscripts " $u$ " and " $t$ " signify the partial derivative by the indicated coordinate.

Introduce the function $\nu=h / u^{3}$ on the subset of $S^{1} \times\left(B^{3}-\{0\}\right)$ where $|z| / \rho<1 / 4$. Where defined, $\nu$ is an analytic functional of $z / u$ which obeys $\nu=z / u+\mathcal{O}\left(|z|^{3} / u^{3}\right)$. Also:

- $|\nu| \leq \zeta \cdot \varepsilon$ on $C^{\prime}$

- $\quad \nu$ tends uniformly to zero as $u$ tends to zero.

With $\nu$ understood, (8.1) is equivalent to the system:

- $\nu_{u}+3 \nu / u-\left(1+\kappa_{1}\right) \cdot \phi_{t}=0$

- $\nu_{t}+\left(1+\kappa_{2}\right) \cdot \phi_{u}=0$

Here, $\kappa_{1}=\rho^{2} / u^{2}-1=\nu^{2}+\mathcal{O}\left(\nu^{4}\right)$ and $\kappa^{2}=g^{2} \cdot \rho^{2} / u^{4}-1=8 \nu^{2}+\mathcal{O}\left(\nu^{4}\right)$ are analytic functionals of $\nu^{2}$ where the latter is less than $1 / 16$.

Step 2 If $C^{\prime}$ is not smooth and $\pi$ is not $1-1$, then $\phi$ and $\nu$ cannot be considered as globally defined functions of $(t, u)$ where $t \in(-\delta, \delta)$ and $u \in\left(0, s_{\varepsilon}\right)$. Rather, they must be thought of as multivalued functions whose values over any point $(t, u)$ specify the $p^{\prime}$ different sheets of $C^{\prime}$ over the $(t, u)$ plane.

The preceding can be said differently as follows: As remarked, the pair $(t, u)$ define local coordinates $C^{\prime}$ except at the points of $\Lambda$. This set $\Lambda$ can be assumed to be infinite or empty, for it $\Lambda$ is finite, one can decrease $s_{0}$ to obtain a $C^{\prime}$ which admits a proper fibration by $\pi$ over. In any event, when $(t, u) \notin \pi(\Lambda)$, then the point $(t, u)$ has a neighborhood that parametrizes $p^{\prime}$ sheets of $C^{\prime}$ via a set, $\left\{\left(\phi, \nu_{1}\right), \ldots\left(\phi_{p^{\prime}}, \nu_{p^{\prime}}\right)\right\}$ of pairs of functions. Here, no two pairs in this set agree at any point of the given neighborhood; and each pair obeys (8.2) and (8.3) on the given neighborhood. However, as $(t, u)$ approaches a point in $\pi(\Lambda)$, two or more pairs from this set approach the same value. What is more, as $(t, u)$ is circled around any point in $\pi(\Lambda)$, monodromy may permute some non-trivial subset of $\left\{\left(\phi, \nu_{1}\right), \ldots\left(\phi_{p^{\prime}}, \nu_{p^{\prime}}\right)\right\}$.

Step 3 The purpose of this step is to prove that

$$
\sup _{j}\left|\nu_{j}\right| \leq c \cdot u
$$

for some constant $c$. The argument for (8.4) starts with (8.3). Differentiate the latter to eliminate $\phi$, and so obtain the following second order equation for $\nu=\nu_{j}$ on the complement of $\pi(\Lambda)$ :

$$
\left(\left(1+\kappa_{1}\right)^{-1}\left(\nu_{u}+3 \nu / u\right)\right)_{u}+\left(\left(1+\kappa_{2}\right)^{-1} \nu_{t}\right)_{t}=0
$$


This is to say that:

$$
\begin{aligned}
& \left(1+\kappa_{1}\right)^{-1} \cdot\left(\nu_{u u}+3 \nu_{u} / u-3 \nu / u^{2}-2 \cdot \kappa_{1}^{\prime} \cdot\left(1+\kappa_{1}\right)^{-1}\left(\nu \nu_{u}^{2}-3 \nu^{2} \nu_{u}\right)\right) \\
& \quad+\left(1+\kappa_{2}\right)^{-1} \cdot\left(\nu_{t t}-\kappa_{2}^{\prime} \cdot\left(1+\kappa_{2}\right)^{-1} \nu \nu_{t}^{2}\right)=0
\end{aligned}
$$

Now consider (8.6) where $\nu \geq 0$ and where $u$ is such that $\sup _{j}\left|\nu_{j}\right| \leq 1 / 32$. Here, (8.6) implies the differential inequality

$$
\left(1+\kappa_{1}\right)^{-1} \cdot\left(\nu_{u u}+3 \nu_{u} / u-3 \nu / u^{2}+\zeta \cdot \nu\right)+\left(1+\kappa_{2}\right)^{-1} \nu_{t t} \geq 0,
$$

where $\zeta$ is a constant which is independent of $u$ and $\nu$.

Note that this last equation holds when $\nu=\nu_{j}$ for each $j$ as long as $\nu_{j} \geq 0$ and $u$ is small. With this last point understood, introduce the function $\nu=$ $\sup \left\{0, \nu_{1}, \ldots, \nu_{j}\right\}$. This function is Lipschitz and it also obeys (8.7), albeit in the weak sense. (In this regard, interpret $\kappa_{1}=\kappa_{1}(\nu)$ and $\kappa_{2}=\kappa_{2}(\nu)$.) With the preceding understood, the point now is to consider applying the maximum principle to obtain an upper bound for $\nu$. For this purpose the constant $\delta$ can be chosen positive and such that:

- $[-\delta, \delta] \subset Z_{R}$,

- $\quad$ Equation (8.7) is valid on a neighborhood of

$$
A \equiv\{(t, u): t \in[-\delta, \delta] \text { and } u \in(0, \delta)\}
$$

- $\quad 0 \leq \nu \leq 1 / 32$ on a neighborhood of $A$,

- $\left|\kappa_{1}\right|+\left|\kappa_{2}\right| \leq 1 / 1000$ on $A$,

- $\delta \leq 1 /(1+4 \cdot \zeta)$, where $\zeta$ appears in (8.7).

With the preceding understood, choose $t_{1} \in(-\delta / 2, \delta / 2)$ and introduce the function

$$
s \equiv 256 \cdot \delta^{-1} \cdot\left(u-u^{2}+\left(t-t_{1}\right)^{2}\right) .
$$

Note that (8.8) implies that

- $\left(1+\kappa_{1}(\nu)\right)^{-1} \cdot\left(s_{u u}+3 s_{u} / u-3 s / u^{2}+\zeta \cdot s\right)+\left(1+\kappa_{2}(\nu)\right)^{-1} \cdot s_{t t} \leq 0$ on $A$,

- $s \geq \nu$ on the boundary of $A$.

It then follows from the comparison principle that $s \geq \nu$ at all points of $A$. And, since $t_{1}$ in (8.9) can be chosen at random in $[-\delta / 2, \delta / 2]$, it follows that

$$
\sup _{j} \nu_{j} \leq 256 \cdot \delta^{-1} \cdot u
$$

at points $(t, u)$ where $t \in[-\delta / 2, \delta / 2]$ and where $u \leq \delta$.

A similar argument proves that $\inf _{j} \nu_{j} \geq-256 \cdot \delta^{-1} \cdot u$ on this same domain. 
Step 4 This step serves up the following technical lemma:

Lemma 8.2 Let $\delta$ and $A$ be as in (8.8). Then the function $\sum_{j}\left(\left|d \phi_{j}\right|^{2}+\left|d \nu_{j}\right|^{2}\right)$ is integrable over the subset of points $(t, u) \in A$ where $t \in[\delta / 2, \delta / 2]$. Moreover, there exists $\zeta \geq 1$ with the following significance: Let $s \in(0, \delta / 8)$ and let $D$ be a disk of radius $s$ in the $(t, u)$-plane with center at a point $\left(t_{1}, u_{1}\right)$ with $\delta / 8 \leq t_{1} \leq \delta / 4$ and $0 \leq u_{1} \leq \delta / 4$. Let $D^{\prime} \equiv D \cap A$. Then

$$
\int_{D^{\prime}} \sum_{j}\left(\left|d \phi_{j}\right|^{2}+\left|d \nu_{j}\right|^{2}\right) \leq \zeta \cdot s^{1 / \zeta}
$$

Proof of Lemma 8.2 To see that $\sum_{j}\left(\left|d \phi_{j}\right|^{2}+\left|d \nu_{j}\right|^{2}\right)$ is locally integrable near points of $\pi(\Lambda)$ argue as follows: Let $w=t+i u$ and let $w_{0} \in \pi(\Lambda)$. Then, the proof of (7.26) can be repeated almost verbatim here to find $\zeta>0$ such that $\sum_{j}\left(\left|d \phi_{j}\right|^{2}+\left|d \nu_{j}\right|^{2}\right) \leq \zeta\left|w-w_{0}\right|^{-2+2 / \zeta}$ on some neighborhood of $w_{0}$.

In order to prove the global integrability of $\sum_{j}\left(\left|d \phi_{j}\right|^{2}+\left|d \nu_{j}\right|^{2}\right)$, first reintroduce the cut-off function $c$ as described prior to (2.1). Then, fix a pair of numbers $s_{0}, s_{1}$ which satisfy $0<s_{0}<s_{1} \leq \delta / 4$. Now, let $\beta$ denote the function $\chi(2|t| / \delta) \cdot \chi\left(u / s_{1}\right) \cdot\left(1-\chi\left(u / s_{0}\right)\right)$. Thus, this function vanishes where either $|t| \geq \delta$ or $u \leq s_{0}$ or $u \geq 2 s_{1}$. On the other hand, this function equals 1 where both $|t| \leq \delta / 2$ and $2 s_{0} \leq u \leq s_{1}$.

Square both sides of (8.3), multiply the result by $\beta^{2}$ and then sum the result over $j$. Next, integrate this sum over $A$. The result, after a judicious application of the triangle inequality and appeals to (8.4) and the fourth point of (8.9), yields

$$
\int_{A} \beta^{2} \sum_{j}\left(\left|d \phi_{j}\right|^{2}+\left|d \nu_{j}\right|^{2}\right) \leq \zeta\left(s_{1} \delta+\left|\int_{A} \beta^{2} \sum_{j}\left(\nu_{j u} \phi_{j t}-\nu_{j t} \phi_{j u}\right)\right|\right) .
$$

Here, $\zeta$ is a constant which is independent of $s_{0}$ and $s_{1}$. Now, integrate by parts on the right-hand side of (8.13) to lift the derivatives off of $\nu_{j}$. The resulting right-hand integal reads:

$$
-2 \int_{A} \sum_{j}\left(\beta_{u} \nu_{j} \beta \phi_{j t}-b_{t} \nu_{j} \beta \phi_{j u}\right)
$$

The fact that $\left\{\left(\phi_{j}, \nu_{j}\right)\right\}$ defines a multivalued function on the complement of $\pi(\Lambda)$ has no effect on the application of integration by parts. Furthermore, there are no anomalous boundary terms in (8.14) from the points in $\pi(\Lambda)$.

These last points can be verified with the following argument: To begin, the data $\left\{\left(\phi_{j}, \nu_{j}\right)\right\}$ can be thought of as the push-forward via $\pi$ of the restriction 
to $C$ of the functions $(\phi, \nu)$. Furthermore, the support of $\pi^{*} \beta^{2}$ on $C$ is the image of a smooth manifold $\Sigma$ with boundary via a map, $f$, which is pseudoholomorphic on the interior of $\Sigma$. This map identifies $C-\pi^{-1}(\Lambda)$ with the complement of a finite number of points in $\Sigma$. Now, note that the integral on the right side of (8.13) is equal to $\int_{\Sigma} f^{*}\left(\pi^{*} \beta^{2} d \nu \wedge d \phi\right)$. Moreover, the functions and forms in this integral are smooth on $\Sigma$, and have support in $\Sigma$ 's interior. Thus, Stokes' theorem applies and equates this integral with $-2 \cdot \int_{\Sigma} f^{*}\left(\nu \pi^{*} d \beta \wedge\right.$ $\left.\pi^{*} \beta d \phi\right)$, which is (8.14).

With (8.14) understood, remark that $|d \beta| \cdot\left|\nu_{j}\right|$ is uniformly bounded courtesy of (8.4). Thus, Holder's inequality implies that (8.14) is no larger in absolute value than

$$
\zeta \cdot\left(s_{1} \cdot \delta\right)^{\frac{1}{2}} \cdot\left(\int_{A} \beta^{2} \sum_{j}\left|d \phi_{j}\right|^{2}\right)
$$

where $\zeta$ is independent of both $s_{0}$ and $s_{1}$. And, this last bound on (8.14) can be substituted into (8.13) to deduce that

$$
\int_{A} \beta^{2} \sum_{j}\left(\left|d \phi_{j}\right|^{2}+\left|d \nu_{j}\right|^{2}\right) \leq \zeta \cdot s_{1} \cdot \delta
$$

where $\zeta$ is, once again, independent of both $s_{0}$ and $s_{1}$.

Since $\zeta$ is independent of both $s_{0}$ and $s_{1}$ in (8.16), one can take $s_{0}$ to 0 in (8.16) to conclude that $\sum_{j}\left(\left|d \phi_{j}\right|^{2}+\left|d \nu_{j}\right|^{2}\right)$ is integrable as asserted in Lemma 8.2. Moreover, (8.16) implies that when $s^{\prime} \leq \delta / 4$, then

$$
\int_{|t| \leq \delta / 4, u \leq s^{\prime}} \sum_{j}\left(\left|d \phi_{j}\right|^{2}+\left|d \nu_{j}\right|^{2}\right) \leq \zeta \cdot s^{\prime} \cdot \delta .
$$

Now consider the bound in (8.12). In this regard, it proves convenient to consider separate arguments for the two cases where the center point $\left(t_{1}, u_{1}\right)$ satisfies either $u_{1} \leq 4 s$ or $u_{1} \geq 2 s$. In the former, case, the integral of $\sum_{j}\left(\left|d \phi_{j}\right|^{2}+\left|d \nu_{j}\right|^{2}\right)$ over $D^{\prime}$ is no greater than its integral over the subset where $\delta / 2 \leq t \leq \delta / 2$ and $u \leq 5 s$. The latter integral is bounded in (8.17) using $s^{\prime}=5 s$.

With the preceding understood, consider the case where $u_{1}>2 s$. In this case, $u>0$ on $D^{\prime}$ and so $C$ is the image of a smooth, pseudo-holomorphic map over $D^{\prime}$. To begin, suppose that $s_{1} \in\left(0, u_{1} / 2\right]$ and let $D \equiv D\left(s_{1}\right)$ denote the disk in the $(t, u)$ plane which is concentric with $D^{\prime}$ but has radius $s_{1}$. Note that the integral of $\sum_{j}\left(\left|d \phi_{j}\right|^{2}+\left|d \nu_{j}\right|^{2}\right)$ over the radius $s_{1}=u_{1} / 2$ disk has been bounded apriori by $\zeta \cdot u_{1} \cdot \delta$. Now, consider squaring both sides of (8.3) and integrating 
over the radius $s_{1}<u_{1} / 2$ disk $D$. The result, after an application of Holder's inequality reads

$$
\int_{D} \sum_{j}\left(\left|d \phi_{j}\right|^{2}+\left|d \nu_{j}\right|^{2}\right) \leq \zeta \cdot\left|\int_{D} \sum_{j} d \nu_{j} \wedge d \phi_{j}\right|+\zeta \cdot s_{1}^{2} .
$$

The next step in the proof of (8.12) analyzes the right-hand side of (8.18) via an integration by parts to obtain the inequality

$$
\int_{D} \sum_{j}\left(\left|d \phi_{j}\right|^{2}+\left|d \nu_{j}\right|^{2}\right) \leq \zeta \cdot\left|\int_{\partial D} \sum_{j \in \Omega^{\prime}} \nu_{j} \cdot d \phi_{j}\right|+\zeta \cdot s_{1}^{2} .
$$

(The arguments which justified (8.14) also justify (8.19).)

Further analysis of the right-hand side of (8.19) requires the following claim: There is a constant $\zeta$ which is independent of $s_{1}$ and of the chosen center point of $D$ such that the right side of (8.19) is no larger than

$$
\zeta \cdot\left(s_{1} \cdot \int_{\partial D} \sum_{j}\left(\left|d \phi_{j}\right|^{2}+\left|d \nu_{j}\right|^{2}\right)+s_{1}^{2}\right) .
$$

when $\partial D \cap \Lambda=\emptyset$. This last claim will be justified momentarily, so accept it for the time being. Then, let $f\left(s_{1}\right)$ denote the integral on the right side of (8.19). Coupled with the preceding claim, (8.19) implies that $f$ satisfies, for all but finitely many values of $s_{1}$, the differential inequality

$$
f \leq \zeta \cdot s_{1} \cdot f^{\prime}+\zeta \cdot s_{1}^{2}
$$

Here, $f^{\prime}$ denotes the derivative of $f$. This last equation can be integrated directly to find that $f\left(s_{1}\right) \leq \zeta \cdot s_{1}^{1 / \zeta}$ where $\zeta$ is independent of $s_{1}$ and of the center point of $D$. (The proof of this last point requires the apriori bound for $f\left(u_{1}\right)$.)

With the preceding understood, the justification of the claim that (8.20) bounds the right-hand side of (8.19) completes the proof of Lemma 8.2. To start this justification, note that as $\partial D$ is assumed to be disjoint from $\Lambda$, the map $\pi$ from $C$ to the $(t, u)$ plane is a $p$-fold covering map near $\pi^{-1}(\partial D)$ and so $\pi^{-1}(\partial D)$ is a smooth, embedded 1 -manifold in $C$. Let $\{\Gamma\}$ denote the set of components of $\pi^{-1}(\partial D)$. Then, the integral on the right side of (8.19) is equal to

$$
\sum_{\Gamma \in\{\Gamma\}} \int_{\Gamma} \nu \wedge d \phi
$$

Now, each boundary component $\Gamma$ is homologically trivial in the $\rho>0$ subspace of $S^{1} \times \mathbb{R}^{3}$ as each deforms to a multiple cover of $\partial D$. This means that

$$
\int_{\Gamma} \nu \wedge d \phi=\int_{\Gamma}(\nu-\alpha) \wedge d \phi
$$

Geometry $8 \mathcal{G}$ Topology, Volume 2 (1998) 
where $\alpha$ is any constant. In particular, take $\alpha=\left(2 \pi \cdot p_{\Gamma} \cdot s_{1}\right)^{-1} \int_{\Gamma} \nu \pi^{*}(d \sigma)$, where $p_{\Gamma}$ is the degree of the covering map $\pi: \Gamma \rightarrow \partial D$ and where $d \sigma$ is the induced Euclidean line element on $\partial D$. (Thus, $\int_{\partial D} d \sigma=2 \pi s_{1}$.) Note that $\pi^{*} d \sigma$ trivializes $T \Gamma$ and so defines a Riemannian metric for $\Gamma$. With respect to this metric, there is a standard eigenvalue estimate which finds that $\int_{\Gamma}(\nu-\alpha)^{2} \pi^{*} d \sigma \leq s_{1}^{2} p^{2} \cdot \int_{\Gamma}|d \nu|^{2} \pi^{*} d \sigma$. Then, with the proceding understood, (8.23) implies that

$$
\sum_{\Gamma}\left|\int_{\Gamma} \nu \wedge d \phi\right| \leq \zeta \cdot s_{1} \cdot \sum_{\Gamma}\left(\int_{\Gamma}|d \nu|^{2} \pi^{*} d \sigma\right)^{\frac{1}{2}}\left(\int_{\Gamma}|d \phi|^{2} \pi^{*} d \sigma\right)^{\frac{1}{2}} .
$$

Meanwhile, the right-hand side of (8.24) is equal to

$$
\zeta \cdot s_{1} \cdot \sum_{j}\left(\int_{\partial D}\left(\left|d \nu_{j}\right| 2\right)^{1 / 2}\left(\int_{\partial D}\left|d \phi_{j}\right|^{2}\right)^{1 / 2} .\right.
$$

Thus, the claim prior to (8.20) follows from (8.24).

Step 5 Let $\delta$ be as in (8.8). The set $\left\{\left(\phi_{j}, \nu_{j}\right)\right\}$ defines a continuous map, $\Phi$, from the set $A^{\prime}=\{(t, u): t \in[-\delta / 8, \delta / 8]$ and $u \in(0, \delta / 8]\}$ into the $p$ 'th symmetric product of $\mathbb{R}^{2}$. This concerns the extension of $\Phi$ to the $u=0$ line in the closure of $A^{\prime}$ :

Lemma 8.3 The map $\Phi$ extends as a continuous map to the $u=0$ line in the closure of $A^{\prime}$. Furthermore, the extension is Holder continuous in the sense that there exist constants $\alpha>0$ and $\zeta$ such that if $w, w^{\prime} \in[-\delta / 8, \delta / 8] \times[0, \delta / 8]$ then the distance from each $(\nu, \phi) \in \Phi(w)$ to $\Phi\left(w^{\prime}\right)$ and from each $(\phi, \nu) \in \Phi\left(w^{\prime}\right)$ to $\Phi(w)$ is no greater than $\zeta \cdot\left|w-w^{\prime}\right|^{\alpha}$.

Proof of Lemma 8.3 First, introduce the function $k=\sup _{j}\left|\phi_{j}\right|$. Then it follows from Lemma 8.2 that $|d k|^{2}$ is integrable over $[-\delta / 4, \delta / 4]$ and that there is a constant $\zeta \geq 1$ with the following significance: Let $s, D$ and $D^{\prime}=D \cap A$ be as in the statement of Lemma 8.2. Then

$$
\int_{D^{\prime}}|d k|^{2} \leq z \cdot s^{1 / \zeta}
$$

With (8.25), a minor modification of the proof of Theorem 3.5.2 in [15] proves that the function $k$ extends to the closure of $A^{\prime}$ as a Holder continuous function in the sense that $\left|k(w)-k\left(w^{\prime}\right)\right| \leq \zeta^{\prime} \cdot\left|w-w^{\prime}\right|^{1 / z^{\prime}}$ when $w, w^{\prime}$ lie in the closure of $A^{\prime}$. Here, $\zeta^{\prime}$ is a fixed constant. (Theorem 3.5.2 in [15] does not consider the case where $k$ is defined on a half space. However, the proof is easily modified to handle this case too. Alternately, one can define $k$ on $[-\delta / 4, \delta / 4] \times[-\delta / 4,-\delta / 4]$ 
by declaring that when $u<0$, then $k(t, u)=k(t,-u)$. This extension of $k$ will obey (8.25) where $D^{\prime}$ is now any disk of radius $\mathrm{s}$ which lies entirely in $[-\delta / 4, \delta / 4] \times[-\delta / 4, \delta / 4]$. Then, Theorem 3.5.2 in [15] applies without modification.)

Now consider the map, $\Psi$, from $\operatorname{Sym}^{n}(\mathbb{C})$ to $\mathbb{C}^{n}$ which sends the unordered set of complex numbers $\left\{\lambda_{1}, \ldots, \lambda_{n}\right\}$ to the coefficients $\left(a_{1}, \ldots, a_{n}\right)$ of the monic, $n$ 'th order polynomial whose roots are $\left\{\lambda_{1}, \ldots, \lambda_{n}\right\}$. (Thus, $a_{j}$ is a homogenous, $j$ 'th order polynomial in the variables $\left\{\lambda_{j}\right\}$. For example, $a_{1}=\sum_{j} \lambda_{j}$ and $a_{n}=\prod_{j} \lambda_{j}$.) This map is a homeomorphism with Holder continuous inverse. This is to say that there exists $\zeta \geq 1$ (which depends on $n$ ) and is such that if $a, a^{\prime} \in \mathbb{C}^{n}$, then the distance from each $\lambda \in \Psi^{-1}(a)$ to $\Psi^{-1}\left(a^{\prime}\right)$ and from each $\lambda \in \Psi^{-1}\left(a^{\prime}\right)$ to $\Psi(a)$ is no greater than $\zeta \cdot\left|a-a^{\prime}\right|^{1 / \zeta}$.

With the preceding understood, the association to each point in $A^{\prime}$ of the element $\Psi\left(\left\{\phi_{j}\right\}\right) \in \mathbb{C}^{n}$ defines a map, $\Psi_{A}$, from $A^{\prime}$ to $\mathbb{C}^{n}$. Because $\sup _{j}\left\{\left|\phi_{j}\right|+\right.$ $\left.\left|\nu_{j}\right|\right\}$ is bounded and because $\Psi$ is polynomial in the $\left\{\phi_{j}\right\}$, it follows from Lemma 8.2 that $\Psi_{A}$ is a Sobolev class $L_{1}^{2}$ map. Furthermore, Lemma 8.2 implies that each component of $\Psi_{A}$ obeys (8.25) for an appropriate choice of $\zeta$. With this understood, the proof of Theorem 3.5 in [15] can be readily modified to prove that $\Psi$ extends to the closure of $A^{\prime}$ as a Holder continuous function. Finally, since $\Psi^{-1}$ is Holder continuous, Lemma 8.3's claim follows.

\section{8.b Assertions 3(b), 3(c) and the $p=1$ case of Assertion 3(a) of Proposition 7.1}

The purpose of this subsection is to establish the $p=1$ version of Assertion 3(a) of Proposition 7.1 as well as Assertions 3(b) and 3(c) of Proposition 7.1 under the assumption that $C$ intersects a neighborhood in $S^{1} \times\left(B^{3}-0\right)$ of the regular point $t_{0}$ as the disjoint union of finite energy, $p=1$ subvarieties.

Here is the argument: Under the given assumption above, $C$ intersects a neighborhood of the point $t_{0}$ in $S^{1} \times\left(B^{3}-0\right)$ as $p$ disjoint components, and so it is sufficient to focus attention on any one component. The latter is described as in Step 1 of subsection 8.a by a pair of functions $(\phi, \nu)$ of the variables $(t, u)$ subject to $(8.2)$ and (8.3) for some suitably small choice of the constant $\varepsilon$. Furthermore, it follows from (8.4) that $|\nu| \leq c \cdot u$ for some constant $c$; and it follows from Lemma 8.3 that $(\phi, \nu)$ extend to the $u=0$ line as Holder continuous functions for some positive Holder exponent. 
Note that the function $\nu$ obeys (8.5), and by virtue of (8.3), the function $\phi$ obeys the equation

$$
u^{-3}\left(\left(1+\kappa_{2}\right) \cdot u^{3} \phi_{u}\right)_{u}+\left(\left(1+\kappa_{1}\right) \cdot \phi_{t}\right)_{t}=0 .
$$

Surprisingly enough, (8.5) and (8.26) can be thought of as equations for functions on a domain in $\mathbb{R}^{5}=\mathbb{R} \times \mathbb{R}^{4}$ as follows: Let $\left(v_{0}, v_{1}, v_{2}, v_{3}, v_{4}\right)$ be Euclidean coordinates on $\mathbb{R} \times \mathbb{R}^{5}$ where $v_{0}$ corresponds to the first factor of $\mathbb{R}$. Then, map $\mathbb{R} \times \mathbb{R}^{4}$ to the $(t, u)$ half plane by writing $t=v_{0}$ and $u=\left(v_{1}^{2}+\ldots v_{4}^{2}\right)^{1 / 2}$. The pull back of $\phi$ and $\nu$ by this map gives functions $\underline{\phi}$ and $\underline{\nu}$, respectively, on $\mathbb{R} \times \mathbb{R}^{4}$. It also proves convenient to introduce the angular coordinate $\theta \in[0, \pi]$ on the $\mathbb{R}^{4}$ factor of $\mathbb{R}^{5}$ by declaring that $\cos \theta=v_{1} / u$. With $\theta$ understood, set $\underline{\lambda} \equiv \underline{\nu} \cos \theta$.

A domain, $D^{\prime}$, for $\underline{\phi}, \underline{\nu}$ and $\underline{\lambda}$ can be taken to be the product of an open line segment centered at zero in the $\mathbb{R}$ factor with the complement of the origin of some small ball in the $\mathbb{R}^{4}$ factor. Let $\varepsilon_{1}$ denote the radii of this segment and this ball. Let $D \subset D^{\prime}$ now denote the product of this same interval with the whole ball in the $\mathbb{R}^{4}$ factor.

Now consider:

Lemma 8.4 Let $\left\{\nabla_{\alpha}\right\}_{\alpha=0, \ldots 4}$ denote the derivatives with respect to the coordinates $\left\{v_{\alpha}\right\}_{\alpha=0, \ldots 4}$ on $\mathbb{R} \times \mathbb{R}^{4}$. Then $\underline{\phi}, \underline{w}$ and $\underline{\lambda}$ all extend from $D^{\prime}$ to $D$ as Holder continuous (with some positive exponent) and Sobolev class $L_{1}^{2}$ functions. Furthermore, $\underline{\phi}$ and $\underline{\lambda}$ obey the equations

- $\Delta \underline{\phi}+\sum_{\alpha, \beta} \nabla_{\alpha}\left(a^{\alpha \beta} \nabla_{\beta} \underline{\phi}\right)=0$

- $\Delta \underline{\lambda}=\nabla_{1} \underline{\kappa}_{1} \nabla_{0} \underline{\phi}-\nabla_{0} \underline{\kappa}_{2} \nabla_{1} \underline{\phi}$.

Here, $\Delta=\sum_{\alpha} \nabla_{\alpha} \nabla_{\alpha}$ is the standard Laplacian on $\mathbb{R}^{5}$ and $a^{\alpha \beta}$ is a diagonal matrix with $a^{00}=\kappa_{1}$ and with $a^{\alpha \alpha}=\kappa_{2}$ for $\alpha>0$.

Proof of Lemma 8.4 The fact that these functions are Sobolev class $L_{1}^{2}$ follows directly from Lemma 8.2. Holder continuity follows from Lemma 8.3. The equation for $\phi$ is simply (8.26), while that for $\underline{\lambda}$ follows from (8.5) with two extra observations: First, $\Delta(\cos \theta)=-3 \cdot \cos \theta / u^{2}$. Second, if $f$ is a function on $\mathbb{R} \times \mathbb{R}^{4}$ of only $t$ and $u$, then $\nabla_{1} f=f_{u} \cdot \cos \theta$.

The equations in (8.27) for $\phi$ and $\underline{\lambda}$ and their initial $L_{1}^{2}$ and Holder bounds can now be used with fairly standard (though tedious) elliptic regularity arguments to prove that the latter are analytic functions on the domain $D$ in $\mathbb{R} \times \mathbb{R}^{5}$. 
(The elliptic regularity arguments are essentially the same as those of Morrey which yield Theorems 6.2.5 and 6.7.6 in [15].)

The analyticity of $\phi$ and $\underline{\lambda}$ imply directly Assertion 3(b) of Proposition 7.1 for the original functions $(\phi, z)$. Then, the analyticity of $(\phi, z)$ and the equations in (8.3) give directly Assertion 3(c) of Proposition 7.1. This analyticity also implies Assertion 3(a) in the $p=1$ case. Indeed, for $\delta>0$ but small, the map $(-\delta, \delta) \times(0, \delta)$ to $S^{1} \times\left(B^{3}-\{0\}\right)$ which assigns the coordinates $(t, x=$ $r \cos \phi, y=\rho \sin \phi, z)$ where $\phi=\phi(t, u), z=z(t, u)$ and $\rho=2^{1 / 2} u \cdot(1+$ $\left.z^{2} / u^{2}\right)$ defines an embedding which is $1-1$ and onto the intersection of $C$ with neighborhood of $t_{0}$.

\section{The number of singular points}

When studying the behavior of $C$ near the singular set $Z_{S} \subset Z$, it is sufficient, after Propositions 5.1 and 7.1, to restrict attention to the case where $C$ is a pseudo-holomorphic subvariety with $n_{ \pm}=0$ at all points of $Z$ and with $q_{ \pm}=0$ at all regular points of $Z$. Furthermore, one can assume that $\mathrm{C}$ has no irreducible components which lie in the $z=0$ half space as all of these are described by Example 1.3.

The results in this section are summarized by:

Proposition 9.1 The set of singular points is finite.

The proof of this proposition occupies the remainder of this section.

Proof of Proposition 9.1 The proposition is an immediate corollary of Proposition 5.2 and Proposition 9.2, below. First, Proposition 5.2 produces a uniform bound by $\zeta \cdot s^{3}$ for the function $\mu(s)$ in (5.1). Then, Proposition 9.2 implies the following claim:

- Let $t_{0} \in Z_{s}$ and let $\nu>0$. If $s$ is small, then the subset of $C$ where $t \in\left[t_{0}-\nu, t_{0}+\nu\right]$ contributes at least $\zeta^{-1} \cdot s^{3}$ to the function $\mu$. Here, $\zeta \geq 1$ is independent of $t_{0}, \nu$, and $s$.

The bound on $\mu$ from Proposition 5.2 plus (9.1) imply that $Z_{S}$ is finite.

As just remarked, Proposition 9.2 leads to (9.1); however a brief digression is required in order to state this proposition. To start the digression, agree to simplify the notation by taking $t_{0}$ to be the origin, 0 , in a Euclidean coordinate 
system for a neighborhood of $t_{0}$ in $Z$. Having done so, fix $\nu>0$ and small so that $Z$ has neither $t=-\nu$ nor $t=\nu$ as singular points. When $r_{1}$ is positive, but small (much less that $\delta$ in (5.1)); and when $s \in\left(0, r_{1}\right)$, let $D(s)$ denote the subset of $S^{1} \times \mathbb{R}^{3}$ where the following constraints hold:

- $t \in[-\nu, \nu]$

- $\rho \leq r_{1}$

- $|z| \leq r_{1}$

- $0 \leq h \leq s$

Remember that when $r_{1}$ is generic and $s$ is small relative to $r_{1}$, then $C$ has empty intersection with the boundary of $D(s)$ where $|z|=r_{1}$. Thus, the intersection of $C$ with the boundary of $D(s)$ consists of the regions where:

- $\quad h=s^{3}$ while $\rho \leq r_{1},|z| \leq r_{1}$ and $t \in[-\nu, \nu]$

- $\quad h=0$ while $\rho \leq r_{1},|z| \leq r_{1}$ and $t \in[-\nu, \nu]$

- $\quad \rho=r_{1}$ while $|z| \leq s^{3} / r_{1}^{2}$ and $t \in[-\nu, \nu]$

- $t= \pm \nu$ while $\rho \leq r_{1},|z| \leq r_{1}$ and $0 \leq h \leq s^{3}$

Use $\partial C$ to denote the intersection of $C$ with the boundary of $D(s)$. Also, use $\partial^{\prime} C$ to denote the intersection of $C$ with the part of the boundary of $D(s)$ where $t= \pm \nu$ and use $\partial^{\prime \prime} C$ to denote the intersection of $C$ with the part of the boundary of $D(s)$ where $\rho=r_{1}$.

Now consider the function

$$
\mu_{0}(s)=\int_{C \cap D(s)} d \phi \wedge d h .
$$

Integration by parts can be used to equate

$$
\mu_{0}(s)=-s^{3} \int_{\partial C \cap\{h=s\}} d \phi-\int_{\partial^{\prime} C} h \cdot d \phi .
$$

End the digression and consider the following proposition:

Proposition 9.2 Suppose that 0 is in $Z_{S}$ and that $q_{+}(0)>0$. When $r_{1}>0$ is small and when $s>0$ is very small, the following is true:

- The first term in (9.5) is greater than $s^{3}$.

- The second term in (9.5) is bounded in absolute value by $\zeta^{\prime} \cdot r_{1} \cdot s^{3}$. Here, $\zeta^{\prime}$ is independent of $s$ and $r_{1}$.

- The third term in (9.5) is bounded in absolute value by $\zeta \cdot s^{6}$. Here, $\zeta$ is independent of $s$ but may depend on $r_{1}$. 
- Thus, $\mu_{0}(s)>s^{3} / 2$ for small $r_{1}$ and $s$.

Note that the fourth assertion of Proposition 9.2 implies (9.1) because when $r_{1}$ is small and $s$ is small, then the set $\Omega(s)$ in (5.1) will contain $D(s)$.

Proof of Proposition 9.2 The proof of this proposition is quite lengthy and so is broken down into steps, fourteen in all.

Step 1 This step proves the second assertion of Proposition 9.2. Here is the argument: Since $\pm \nu$ are regular points, Proposition 7.1 finds $r_{0}>0$ and a constant $\zeta$ such that $|d \phi| \leq \zeta$ on $\partial C^{\prime}$ when $r_{1} \leq r_{0}$. Thus, since $h \leq s^{3}$, the second assertion of Proposition 9.2 follows by integration.

Step 2 This step states and proves Lemma 9.3, below, which implies the third assertion of Proposition 9.2. Note that Lemma 9.3 also plays a pivotal role in the proof of Proposition 9.2's first assertion.

Lemma 9.3 There exists $r_{0}>0$ such that the following is true: Given $r_{1} \in$ $\left(0, r_{0}\right]$, let $I\left(r_{1} ; s\right)=\int_{\partial^{\prime \prime} C}|d \phi|$. Then, for all but finitely many values for $r_{1} \in\left[r_{0} / 2, r_{0}\right]:$

- The function $s^{-3} \cdot I(r ; s)$ is bounded as s tends to zero.

- The quantity $s^{-3}$. length $\left(\partial^{\prime \prime} C\right)$ is bounded as $s \rightarrow 0$.

- The number of components of $\partial^{\prime \prime} C$ becomes constant as $s \rightarrow 0$. Furthermore, each component of $\partial^{\prime \prime} C$ is an interval on which $d h \neq 0$ when $s$ is small.

Proof of Lemma 9.3 Since $|d \phi| \leq r_{1}^{-1}$ on $\partial^{\prime \prime} C$, the size of $I(s)$ will be $\mathcal{O}\left(s^{3}\right)$ when length $\left(\partial^{\prime \prime} C\right)$ is also $\mathcal{O}\left(s^{3}\right)$. Thus, the first assertion follows from the second. A uniform bound for $s^{-3}$. length $\left(\partial^{\prime \prime} C\right.$ ) will occur (for small $s$ ) if $C$ intersects the constraint surface where $r=r_{1}$ and $h=0$ in smooth points, and if $d h \neq 0$ at all points of this intersection.

The fact that $r_{1}$ can be chosen to make the latter true follows from:

Lemma 9.4 There are at most finitely many singular points of $C$ in any compact subset of $C$. In addition, there are at most finitely many critical points of the restriction of $h$ to the smooth part of any compact subset of $C$.

Given Lemma 9.4, one can choose $r_{1}$ from the complement of a finite set to insure that $d h \neq 0$ where $h=0$ and $r=r_{1}$. This makes both the second 
and third points of Lemma 9.3 true. (The final point follows from the implicit function theorem when $d h \neq 0$ at the points in $C$ where $h=0$ and $r=r_{1}$.)

Proof of Lemma 9.4 The first assertion about the singularities of $C$ follows from general regularity results about pseudo-holomorphic submanifolds. Indeed, one can parametrize $C$ using the smooth model curve $C_{0}$ and the pseudo-holomorphic map $f: C_{0} \rightarrow C$ as in Definition 1.1. Then, the result follows from general regularity results about $f$ as in [17], [24] or [14].

As for the statement about $h$, consider $f$ and $C_{0}$ as above and consider the pull-back $f^{*} h$. This satisfies an elliptic equation on $C_{0}$ with the schematic form $d^{*} d\left(f^{*} h\right)+a \cdot d\left(f^{*} h\right)=0$, where $a$ is an appropriate vector field on $C_{0}$. Indeed, because $f$ is pseudo-holomorphic, this follows from the fact that the restriction of $h$ to the smooth part of $C$ obeys the equation $d\left(\left(g \rho^{2}\right)-1 \cdot J d h=0\right.$. It then follows using the unique continuation theorem of Aronzajn [1] as applied to $f^{*} h$ that the latter can have only finitely many critical points in any compact subset of $C_{0}$. (Argue here as in the proof of Lemma 7.5.)

Step 3 This step begins the proof of the first assertion of Proposition 9.2. In this regard, the simplest case occurs when the intersection of $\partial C$ with the surface $h=s^{3}$ contains a closed loop. Then, the first term in (9.5) is nonnegative and has the form $2 \pi s^{3} \cdot m$, where $m$ is a positive integer because $(2 \pi)^{-1} \cdot d \phi$ restricts to $\partial C$ to define an integral valued cohomology class. (The possibility that $m=0$ is ruled out with the observation that in this case, either $q_{+}=0$ or else $C$ is identical to a surface from either Example 1.3 or 1.4.)

Note that the argument for the first assertion of Proposition 9.2 in the general case also involves a cohomological argument using $d \phi$. However, in the general case, these cohomological arguments are coupled with certain analytic results. The first of these analytic results concerns some special properties of the level sets of $h$ on $C$ and is discussed here.

To begin, choose $r_{1}>0$ and small so that the conclusions of Lemma 9.3 hold. Fix $s^{\prime} \in(0, s]$ for the moment and consider the subset of $C \cap D(s)$ where $h=s^{\prime 3}$. When $s^{\prime}$ is chosen from a certain dense and open set, then $h^{-1}\left({s^{\prime}}^{3}\right) \cap(C \cap D(s))$ will lie in the smooth portion of $C$ and it will miss all critical points of $h$. In this case, the set

$$
\gamma\left(s^{\prime}\right)=h^{-1}\left(s^{\prime 3} \cap(C \cap D(s))\right.
$$

is a smoothly embedded curve. Furthermore, if $d h$ is used to orient the normal bundle to $\gamma\left(s^{\prime}\right)$, then the restriction of $d \phi$ to $\gamma\left(s^{\prime}\right)$ is nowhere vanishing and negative definite. Thus, one has the following crucial observation: 
- The integral of $-d \phi$ over $\gamma\left(s^{\prime}\right)$ is bounded from below by its integral over any subset of $\gamma\left(s^{\prime}\right)$.

Step 4 The following lemma is a key corollary to Lemma 9.3:

Lemma 9.5 Suppose that $r_{1}>0$ is chosen as in Lemma 9.3, but small; and suppose that $s>0$ is very small. Let $s^{\prime} \in(0, s]$ be such that $-\gamma\left(s^{\prime}\right) d \phi>5 / 9$. Then $-\int_{\gamma\left(s^{\prime}\right)} d \phi>1 / 2$ for all $s^{\prime \prime} \in(0, s]$. In particular, $\mu_{0}\left(s^{\prime \prime}\right)$ from (9.5) satisfies $\mu_{0}\left(s^{\prime \prime}\right)>s^{\prime \prime 3} / 2$ for all such $s^{\prime \prime}$.

Proof of Lemma 9.5 The proof starts by connecting the curve $\gamma\left(s^{\prime}\right)$ to $\gamma\left(s^{\prime \prime}\right)$ to form a closed curve. However, this task requires a digression to consider the endpoints of $\gamma\left(s^{\prime}\right)$. To start the digression, note that $\gamma\left(s^{\prime}\right)$ will have endpoints in $\partial^{\prime} C$ and in $\partial^{\prime \prime} C$. To consider the endpoints of $\gamma\left(s^{\prime}\right)$ in $\partial^{\prime} C$, remark first that the $t=\nu$ portion of $\partial^{\prime} C$ consist of some number $p \geq 0$ of arcs, each with one endpoint in $Z$ and the other endpoint where $h=s^{3}$. Furthermore, if $r_{1}$ is small and s is very small, then the $h=s^{3}$ endpoint of an arc component of $\partial^{\prime} C$ does not sit in $\partial^{\prime \prime} C$ as $C$ is assumed to have no $z=0$ components. With $\partial^{\prime} C$ understood, then the boundary of $\gamma\left(s^{\prime}\right)$ in the $t=\nu$ portion of $\partial^{\prime} C$ will consist of $p$ points, each on one of the corresponding arc components of $\partial^{\prime} C$. Thus, the endpoints of $\gamma\left(s^{\prime}\right)$ and $\gamma\left(s^{\prime \prime}\right)$ in the $t=\nu$ portion of $\partial^{\prime} C$ are paired by the condition that they lie in the same component arc of $\partial^{\prime} C$. Then, the intervening segment of this component arc can be used to connect these paired points.

Now consider the endpoints of $\gamma\left(s^{\prime}\right)$ in $\partial^{\prime \prime} C$. As the latter is a collection of arcs where $d h \neq 0$, there is a point of $\gamma\left(s^{\prime}\right) \cap \partial^{\prime \prime} C$ in each such arc, and thus a natural pairing between the points of $\gamma\left(s^{\prime}\right) \cap \partial^{\prime \prime} C$ and $\gamma\left(s^{\prime \prime}\right) \cap \partial^{\prime \prime} C$ with an arc in $\partial^{\prime \prime} C$ connecting the paired points.

With the preceding understood, connect the endpoints of $\gamma\left(s^{\prime}\right)$ in $\partial^{\prime} C$ with those of $\gamma\left(s^{\prime \prime}\right)$ by arcs in $\partial^{\prime} C$ and likewise connect the endpoints of $\gamma\left(s^{\prime}\right)$ with those of $\gamma\left(s^{\prime \prime}\right)$ by arcs in $\partial^{\prime \prime} C$. Think of the result as a 1 -cycle in $C$. (This cycle traverses $\gamma\left(s^{\prime}\right)$ in the positive direction and $\gamma\left(s^{\prime \prime}\right)$ in the negative direction.) This 1-cycle in $C$ is null homologous as one can consider the homology which moves $s^{\prime}$ to $s^{\prime \prime}$. Thus, $-d \phi$ has integral zero over this cycle. Then, the contribution to the integral of $-d \phi$ over the portion of the 1 -cycle in $\partial^{\prime} C$ is uniformly small $\left(\mathcal{O}\left(r_{1}\right)\right.$, courtesy of Proposition 7.1), and that over the portion in $\partial^{\prime \prime} C$ is also small $(\mathcal{O}(s)$, courtesy of Lemma 9.3). Thus, the contributions to the integral from $\gamma\left(s^{\prime}\right)$ and $\gamma\left(s^{\prime \prime}\right)$ must be almost the same. 
Step 5 The first assertion of Proposition 9.2 follows immediately using Lemma 9.5 with the following:

Lemma 9.6 Choose $r_{1}>0$, but small so that the conclusions of Lemma 9.3 hold. Suppose that $s$ is sufficiently small. Then, there exists $s^{\prime} \in(0, s]$ and an arc, $\gamma$, which lies in $C \cap D(s) \cap\left\{h=s^{\prime 3}\right\}$ and which obeys $-\int_{\gamma} d \phi>\pi / 2$. Then, because $-d \phi>0$ on the set $C \cap\left\{h=s^{\prime 3}\right\}$, it follows that $-\int_{\gamma\left(s^{\prime}\right)} d \phi>\pi / 2$ as well.

Proof of Lemma 9.6 The proof of this lemma occupies the remaining steps in the proof of Proposition 9.2.

Step 6 This step constitutes a digression of sorts to state an auxilliary result which will be used later:

Lemma 9.7 Either $h=$ constant on $C$, or else there exists $N<\infty$ with the following significance: Let $X$ denote the subset of $S^{1} \times\left(B^{3}-\{0\}\right)$ where $\left(x^{2}+y^{2}+z^{2}\right)^{1 / 2}<1 / 2$. Then $N$ bounds the intersection number of $C$ with any pseudo-holomorphic curve $S$ on which $h$ is constant.

Proof of Lemma 9.7 The proof starts with a standard observation:

- Let $K$ be a compact subset of $S^{1} \times\left(B^{3}-\{0\}\right)$. Then $C$ has only finitely many intersections with any $h=$ constant pseudo-holomorphic curve $\Sigma$ in $K$.

One can use (9.8) to draw the following conclusion: Given $\varepsilon>0$, there exists $N_{\varepsilon}$ such that $C$ has intersection number $N_{\varepsilon}$ or less in $X$ with any $h=$ constant pseudo-holomorphic curve where $|h|>\varepsilon$. So, at issue are the $h=$ constant curves with small $|h|$.

To consider the case of an $h=$ constant curve with small $|h|$, first use (9.8) with the fact that $h$ is continuous on $C$ to draw the following conclusion: Unless $h \equiv 0$ on $C$, there exists $\varepsilon>0$ and an open interval $I \subset(1 / 2,1)$ such that $|h|>\varepsilon$ at all points of $C$ where $r \equiv\left(x^{2}+y^{2}+z^{2}\right)^{1 / 2} \in I$. This last remark is equivalent to the assertion that $C$ has empty intersection with all curves $\Sigma$ with $h=$ constant at points where $r \in I$ if the constant in question has norm less than $\varepsilon$.

With these last points understood, use (9.8) with the preceding remark to conclude that unless $h=$ constant on $C$, then $C$ has constant intersection number with all pseudo-holomorphic curves $\Sigma$ where $h=$ constant $\in(0, \varepsilon)$. Conclude 
likewise that unless $h=$ constant on $C$, then $C$ has constant intersection number with all pseudo-holomorphic curves where $h=$ constant $\in(0,-\varepsilon)$. Thus, the only $h=$ constant curve where $C$ might have unbounded intersection number is with some $h=0$ pseudo-holomorphic curve. However, if $C$ has more than $N$ intersection points with such a curve, then $C$ will have more than $N$ such with any $h=$ constant pseudo-holomorphic curve if $|h|$ is sufficiently small.

Step 7 Now return to the proof of Lemma 9.6. This step summarizes some consequences of the results from Sections 3 and 4 together with:

Lemma 9.8 Given $\varepsilon>0$ and $\alpha>0$, there exists $s_{0}>0$ such that when $s \in\left(0, s_{0}\right)$, then the following is true: The intersection of $C$ with the set of points where $t^{2}+\left(\rho^{2}+z^{2}\right) \leq s^{2}$ and $\alpha|t| \leq\left(\rho^{2}+z^{2}\right)^{1 / 2}$ is the disjoint union $C_{\rho} \cup C_{z}$, where:

- $|z| / \rho<\varepsilon$ on $C_{\rho}$ and $\rho /|z|<\varepsilon$ on $C_{z}$.

- If $C^{\prime}$ denotes either $C_{\rho}$ or $C_{z}$, then $\int_{C^{\prime}} d \phi \wedge d h \leq \varepsilon s^{3}$.

- The functions $t$ and $u=(-f)^{1 / 2}$ restrict to a neighborhood of all but a set of at most countably many points in $C_{z}$ as local coordinates. This countable set, $\Lambda$, consists of the singular points of $C_{z}$ and the critical point of $d t$ (or equivalently $d u$ ). This set has no accumulation points in $C_{z}$.

- The map $\pi$ to the $(t, u)$ plane given by restriction of $t$ and $u$ to $C_{z}$ has the following additional property: Given $\varepsilon, \alpha$ and $s$, there exists $\nu_{0}$ such that when $\nu \in\left(0, \nu_{0}\right)$, then $\pi$ maps onto the set $A$ where $\alpha|t|<u<\nu$. In addition, when $(t, u) \in A-A \cap \pi(\Lambda)$, then $\pi^{-1}(t, u)$ has precisely $q_{+}(0)$ elements.

Proof of Lemma 9.8 The first two assertions follow directly from Proposition 3.3. The third assertion is proved by copying verbatim the proof of Lemma 7.5. The existence of $\tau_{0}$ which makes the fourth assertion true follows from Propositions 3.2 and 4.1 .

Step 8 Let $C_{z}$ be as in Lemma 9.8 with $\varepsilon, \alpha$ and $s$ all small. (Some precise bounds will be given subsequently.) Then, by definition, the functions $t$ and $u=\left(z^{2}-\rho^{2} / 2\right)^{1 / 2}$ restrict as coordinate functions near any point of $C_{z}-\Lambda$. This is to say that each such smooth point has a neighborhood which is specified by writing 
- $\quad x=x(t, u), y=y(t, u)$

- $z^{2}=u^{2}+2^{-1} \cdot\left(x^{2}+y^{2}\right)$.

As in the proof of the second assertion of Proposition 7.2, the $\mathbb{C}$-valued function $\eta=x+i \cdot y$ is constrained to obey (7.9) with $a$ and $b$ obeying the bounds $|a|+|b| \leq \zeta \cdot|\eta|^{2} / u^{2}$. In particular, according to Lemma 9.8, one has $|a|+|b| \leq \varepsilon$ when $\varepsilon$ is small.

As with the discussion in Section 7, the parametrization in (9.9) is only local in the following sense: The association of the functions $(t, u)$ to points in $C_{z}$ defines a map $\pi$ to the $(t, u)$ plane which is $q^{\prime}$ to 1 where the number $q^{\prime}=q_{+}(0)$. Thus, where (9.11) holds, $C$ is at best described by a multivalued map from the $(t, u)$ plane to $\mathbb{C}$. Put differently, the portion of $C$ where $(9.11)$ holds is described globally by a map from the indicated domain in the $(t, u)$ plane into the $\operatorname{Sym}^{q^{\prime}} \mathbb{C}$. The coordinates of the latter map consist of a $(t, u)$ dependent, unordered set of complex numbers $\left\{\eta_{j}\right\}$; and each $\eta \in\left\{\eta_{j}\right\}$ is a smooth function which obeys (7.9) where it is unambiguously defined. The set $\left\{\eta_{j}\right\}$ describe the sheets of $C_{z}$ over the image of $\pi$. Alternately, one can think of $\left\{\eta_{j}\right\}$ as the $\pi$-push-forward of the function on $C$ which assigns to the value of $x+i \cdot y$ to a point.

With the preceding understood, let $\theta \equiv \prod_{j} \eta_{j}$. The domain of $\theta$ can be taken to be any small $\alpha, \varepsilon$ and $\nu$ version of the subset $A$ of Lemma 9.8. Here, $\theta$ is a continuous function which is smooth away from $\pi(\Lambda)$.

As in Section 7, when $\alpha, \varepsilon$ and then $\nu$ are chosen and small, the function $\theta$ obeys (7.12) and (7.13) on $A$. Moreover, the proof of Lemma 7.7 can be repeated more or less verbatim (using Lemma 7.8 to control $m$ instead of Lemma 7.6) to write $\theta$ as

$$
\theta=\kappa^{-1} \theta^{\prime}
$$

where $\theta^{\prime}$ obeys $\partial \theta^{\prime}=0$ on its domain of definition; and where $\kappa$ is described in the three points of Lemma 7.7. (As in Section $7, \partial=2^{-1} \cdot\left(\partial_{t}-i \cdot \partial_{u}\right)$.)

To be more precise, Lemma 7.7 writes $\kappa=u^{q^{\prime} / 2} \cdot e^{\tau}$, where $\tau$ obeys

$$
\partial \tau+i \cdot \chi_{*} \cdot m / 4 u=0
$$

where $\chi_{*}$ is a suitable cut off function with values in $[0,1]$ which equals one on the domain in $A$ in Lemma 9.8 and which vanishes where $|t| \geq 2 u / \alpha$ and $u \geq 2 \nu$. Otherwise, the precise details of $\chi_{*}$ are not relevant to the subsequent discussion. 
Step 9 For almost all choices of $v \in(0, \nu)$, the arc $a(v)$ in the $(t, u)$ plane where $\left(t^{2}+u^{2}\right)^{1 / 2}=v$ and where $\alpha|t| \leq u$ will miss $\pi(\Lambda)$. For such $v$, the $\pi$-inverse image of the $\operatorname{arc} a(v)$ is a smooth submanifold in $C$.

Suppose that $v$ is such that $a(v)$ misses $\pi(\Lambda)$ and consider the integral of the imaginary part of $-d \theta^{\prime} / \theta^{\prime}$ over $a(v)$. Let $L(v)$ denote this integral. To estimate this integral, first write $\theta^{\prime}=(t+i \cdot u)^{q^{\prime}} \cdot \theta^{\prime \prime}$. Then

$$
L(v) \geq q^{\prime} \cdot \pi-\zeta \cdot \alpha-\int_{a(v)} \operatorname{im}\left(d \theta^{\prime \prime} / \theta^{\prime \prime}\right) .
$$

Here, the constant $\zeta$ is independent of $v$ and $\alpha$ as long as both are small.

To estimate the right most term in (9.12), introduce polar coordinates $v=$ $\left(t^{2}+u^{2}\right)^{1 / 2}$ and $\sigma=\tan ^{-1}(u / t)$ in the $(t, u)$ plane. Also, agree to write $\theta^{\prime \prime}=e^{\Delta}$, where $\Delta$ is also annihilated by $\partial$. Then, write $\Delta=\Delta_{1}+i \cdot \Delta_{2}$ where $\Delta_{1}$ and $\Delta_{2}$ are real. (Note that $\theta^{\prime \prime}$ is not zero on $A$ when $\delta$ is small - this is guarranteed by Lemma 9.7.) With the preceding understood, the right most term in (9.12) is given by

$$
L_{1}(v) \equiv-\int_{a(v)} \partial_{\sigma} \Delta_{2} d \sigma
$$

Since $\partial \Delta=0$, one has

$$
v^{-1} L_{1}(v)=\int_{a(v)} \partial_{v} \Delta_{1} d \sigma
$$

Given (9.14), take a pair of numbers, $0<v^{\prime}<v^{\prime \prime}<\nu$ and integrate $v^{-1} L_{1}(v)$ over the interval $\left[v^{\prime}, v^{\prime \prime}\right]$. The result, according to (9.14), is

$$
\int_{\left[v^{\prime}, v^{\prime \prime}\right]} v^{-1} \cdot L_{1}(v) \cdot d v=\int_{a\left(v^{\prime \prime}\right)} \Delta_{1} d \sigma-\int_{a\left(v^{\prime}\right)} \Delta_{1} d \sigma
$$

The claim now is that if $v^{\prime}$ is sufficiently small, then the right-hand side of (9.15) is positive. To see that such is the case, use the condition $|\eta| / z \leq \varepsilon$ to see that $|\eta| / u<\varepsilon /\left(1-\varepsilon^{2} / 2\right)$ on $\left.C\right|_{A}$. Then, use the points in Lemma 7.7 to conclude that $\left|\theta^{\prime \prime}\right| \leq u^{1 / 4}$ on its domain of definition. Since $\theta^{\prime \prime}$ does not vanish when $\nu$ is small (due to Lemma 9.7), it follows that, given $v^{\prime \prime}>0$, there exists $v^{\prime}>0$ such that the supremum of $\Delta_{1}$ where $v \in\left(0, v^{\prime}\right)$ is strictly less than the infimum of $\Delta_{1}$ where $v=v^{\prime \prime}$.

Given that there exists $v^{\prime}>0$ where (9.15) is positive, there exists $v>0$ and as small as desired, where $L_{1}(v)>0$. For such $v$, the number $L(v)$ in (9.11) obeys the bound $L(v)>\left(q^{\prime}-1 / 100\right)$. 
Step 10 Let $a^{\prime}(v)=\pi^{-1}(a(v)) \subset C$. Then, $L(v)$ is not equal to $-\int_{a^{\prime}(v)} d \phi$, but almost so. In fact, it follows from (7.22) that $-\int_{a^{\prime}(v)} d \phi$ differs from $L(v)$ by no more than a uniform multiple of $\varepsilon \cdot|\ln (\alpha)|$. The argument for this is as follows: First of all, the integral of $-d \phi$ over $a^{\prime}(v) \equiv \pi^{-1}(a(v))$ is equal to that of $\pi_{*}(-d \phi)$ over $a(v)$. Then, the difference between the $L(v)$ and the integeral of $\pi_{*}(-d \phi)$ over $a(v)$ is proportional to the integral over $a(v)$ of $-d(i m(\tau))$. To estimate this last integral, remark first that $\tau(\cdot)$ can be written as in (7.18) except that $\chi_{*} \cdot m$ should replace $m$ inside the integral. With the preceding understood, let $\chi$ be a standard, non-increasing cut-off function on $[0, \infty)$ which takes value 1 on $[0,1]$ and 0 on $[2, \infty)$. Then, decompose the $\chi_{*} \cdot m$ version of (7.22) into a sum of two integrals; these being:

$$
\begin{aligned}
& \tau_{1}^{\prime}=-i(2 \pi)^{-1} \int_{u^{\prime} \geq 0} \chi\left(\left|w-w^{\prime}\right| / 2 u\right) \chi_{*} m\left(w^{\prime}\right) /\left(\left(\bar{w}-\bar{w}^{\prime}\right) \cdot\left(\bar{w}-w^{\prime}\right)\right) d \bar{w}^{\prime} \wedge d w^{\prime} \\
& \tau_{2}=-i(2 \pi)^{-1} \int_{u^{\prime} \geq 0}\left(1-\chi\left(\left|w-w^{\prime}\right| / 2 u\right)\right) \chi_{*} m\left(w^{\prime}\right) /\left(\left(\bar{w}-\bar{w}^{\prime}\right) \cdot\left(\bar{w}-w^{\prime}\right)\right) d \bar{w}^{\prime} \wedge d w^{\prime}
\end{aligned}
$$

Now, note that $\left|\tau_{1}\right| \leq \zeta \varepsilon$ for some constant $\zeta$ which is independent of $\varepsilon, \alpha$ and $\nu$. This follows using Lemma 7.8 and the following:

- $\quad\left|w-\bar{w}^{\prime}\right| \geq u$ when $w$ and $w^{\prime}$ lie in the upper half plane.

- $\left|w-w^{\prime}\right| \geq 4 u$ is required for a non-zero contribution.

The details here are identical to those in Section 7 which establish the $\zeta \varepsilon$ bound for (7.19).

As for $\tau_{2}$, the claim here is that

$$
\left|d \tau_{2}\right| \leq \zeta \cdot \varepsilon / u,
$$

where $\zeta$ is independent of $\varepsilon, \alpha$ and $\delta$. Indeed, this follows readily by differentiating the second line of (9.16) and again invoking Lemma 7.8. In this case, argue by breaking the integration region into annuli as described in the derivation of the $\zeta \ln (\varepsilon)$ bound for (7.20) in Section 7 .

Given the preceding, it follows from (9.18) and the estimate $\left|\tau_{1}\right|<\zeta \cdot \varepsilon$ that

$$
\left|\int_{a(v)} d \tau\right| \leq \zeta \cdot\left(\sup _{a(v)}\left|\tau_{1}\right|+\varepsilon \cdot \int_{a(v)} u^{-1} \leq z \cdot(\varepsilon+\varepsilon \cdot|\ln (\alpha)|) .\right.
$$

Step 11 This step digresses momentarily to justify a simplifying assumption. The digression begins with the observation that the subsequent arguments for Lemma 9.6 need only consider the case where, for small $s^{\prime}$, the corresponding 
$\gamma\left(s^{\prime}\right)$ (as defined in (9.6)) has no circle component. Indeed, as already remarked in Step 3, if there is a circle component, the integeral of $-d \phi$ over this component is a positive, integral multiple of $2 \pi$ since $-d \phi$ restricts to $\gamma\left(s^{\prime}\right)$ as a volume form. Thus, it is sufficient to make the following assumption: Let $s^{\prime} \in(0, s]$ and suppose that the corresponding $\gamma\left(s^{\prime}\right)$ misses the critical points of $h$ on $C$ and the singular points of $C$. Then $\gamma\left(s^{\prime}\right)$ is a finite union of disjoint, embedded arcs with endpoints on $\partial^{\prime} C$.

Step 12 Choose $v>0$ and small where $-\int_{a^{\prime}(v)} d \phi>\left(q^{\prime}-1 / 100\right)$. A slight (and arbitrarily small) change of $v$ will insure that $a^{\prime}(v)$ misses all critical points of $h$ on $C$, and this condition will henceforth be assumed. Additional constraints on $v$ are as follows: First, by choosing $v$ small, the points in $C$ where $\left(t^{2}+u^{2}\right)^{1 / 2}=v$ and $t=-u / \alpha$ will assuredly lie in $C \cap D(s)$ on level curve $\left\{h=s_{1 j}\right\}_{1 \leq j \leq q^{\prime}}$ with each $s_{1 j}<s$. Furthermore, after possibly a slight change of $v$, each of the corresponding level sets $\left\{h^{-1}\left(s_{1 j}\right)\right\}$ misses all singular points of $C$, and each $\left\{s_{1 j}\right\}$ is a regular value of $h$. This implies that each level set $h=s_{1 j}$ is a smoothly embedded curve, $\gamma\left(s_{1 j}\right)$, in the smooth part of $C$. Likewise, no generality is lost by assuming that the points in $C$ where both $\left(t^{2}+u^{2}\right)^{1 / 2}=v$ and $t=u / \alpha$ lies in $C \cap D(s)$ on level curves $\left\{h=s_{2 j}\right\}$ with $s_{2 j}<s$, and where each such level curve is a smoothly embedded curve, $\gamma\left(s_{2 j}\right)$, in the smooth part of $C$.

There is some direction on each $\gamma\left(s_{1 j}\right)$ where $-\phi$ is increasing, and likewise on each $\gamma\left(s_{2 j}\right)$. Thus, $a(v)$ can be completed by attaching the relevant portions of $\gamma\left(s_{1 j}\right)$ and $\gamma\left(s_{2 j}\right)$ to a give a piecewise smooth arc in $C \cap D(s)$, where each endpoint lies either in $\partial^{\prime} C$ or $\partial^{\prime \prime} C$, and over which the integral of $-d \phi$ is at least $\left(q^{\prime}-1 / 100\right) \cdot \pi$. Note that this arc has at most $q^{\prime}$ components, so there is at least one component, say $\gamma^{\prime}$, with $\int_{\gamma^{\prime}}(-d \phi)>15 \cdot \pi / 16$.

Step 13 The construction of $\gamma^{\prime}$ does not complete the proof of Lemma 9.6 because $\gamma^{\prime}$ does not necessarily lie in a level set of $h$. To achieve the latter requirement, it is necessary to modify $\gamma^{\prime}$. This modification is carried out via a Morse theoretic argument on $C$ which uses the function $h$ as the Morse function and uses the level sets of $\phi$ as the flow lines of a pseudo-gradient vector field. However, before starting, remark that the argument that follows is complicated by three technical issues:

- $C$ may have some singular points.

- $h$ may have degenerate critical points.

- The pseudo-gradient flow lines may not be in general position. 
The first issue is dealt with by pulling back the story to the universal model $C_{0}$ via the pseudo-holomorphic map $f: C_{0} \rightarrow S^{1} \times\left(B^{3}-\{0\}\right)$ as described in Definition 1.1. The second and third issues in (9.20) will be dealt with by considering small perturbations of both $f^{*} h$ and $f^{*} \phi$.

In any event, to begin, choose $s_{0}>0$ but less than the infimum of $h$ on $\gamma^{\prime}$ and so that $\gamma\left(s_{0}\right)$ avoids all critical points of $h$ and all singularities of $C$. Let $f: C_{0} \rightarrow S^{1} \times \mathbb{R}^{3}$ denote the smooth model for $C$ from Definition 1.1. Let $D_{0}=f^{-1}\left(D(s) \cap\left\{h \geq s_{0}\right\}\right)$. Thus, the boundary of $D_{0}$ consists of a finite union of arcs, where each arc lies either in a component of $f^{-1}\left(\partial^{\prime} C \cap\left\{h \geq s_{0}\right\}\right)$ or a component of $f^{-1}\left(\partial^{\prime \prime} C \cap\left\{h \geq s_{0}\right\}\right)$ or else in a level set of $f^{*} h$ with level either $s_{0}$ or $s$. (The latter coincide with $f^{-1}\left(\gamma\left(s_{0}\right)\right)$ and $f^{-1}(\gamma(s))$, respectively.)

On the complement of the critical set of $f^{*} h$, the tangents to the level sets of $f^{*} \phi$ define a vector field which is pseudo-gradient like for the function $f^{*} h$. Note that $f^{-1}\left(\partial^{\prime} C\right)$ may not be a union of level sets of $f^{*} \phi$, but Proposition 7.1 implies that no generality is lost by requiring $f^{*} h$ to restrict to $f^{-1}\left(\partial^{\prime} C\right)$ without critical points. Meanwhile, the requirements for the chosen s insure that $f^{*} h$ also restricts to $f^{-1}\left(\partial^{\prime} C\right)$ without critical points. Thus, one can choose a pseudo-gradient for $f^{*} h$ which is tangent to the level sets of $f^{*} \phi$ except in some arbitrarily small, but apriori fixed neighborhood of $f^{-1}\left(\partial^{\prime} C\right) \cup f^{-1}\left(\partial^{\prime \prime} C\right)$.

The simplest case to consider assumes that $f^{*} h$ has only non-degenerate critical points and that the tangents to the level sets of $f^{*} \phi$ give a suitably generic pseudo-gradient for $f^{*} h$. This genericity assumption will be enforced in the remainder of this step; the general case is considered in Step 14.

Under the preceding genericity assumption, it follows by standard Morse theoretic considerations that $D_{0}$ has a 1 -skeleton which is constructed as follows: Start with the arcs of $\left(f^{*} h\right)^{-1}\left(s_{0}\right)$ and add a finite set of arcs in level sets of $f^{*} \phi$ whose endpoints lie on the arcs of $\left(f^{*} h\right)^{-1}\left(s_{0}\right)$.

This last picture of the one skeleton of $D_{0}$ implies that there is a continuous, embedded arc, $\lambda_{1}$, in $D_{0}$ with the following properties:

- $\lambda_{1}$ is a union of components, each of which is either an arc in $f^{-1}\left(\gamma\left(s_{0}\right)\right)=$ $\left(f^{*} h\right)^{-1}\left(s_{0}\right)$ or else an arc in a level set of $f^{*} \phi$.

- The boundary points of $\lambda_{1}$ lie in $f^{-1}\left(\partial^{\prime} C \cap \partial^{\prime \prime} C \cap\left\{h=s_{0}\right\}\right)$ and each boundary point of $\gamma^{\prime}$ is connected by an arc in $f^{-1}\left(\partial^{\prime} C \cap \partial^{\prime \prime} C \cap\left\{h \geq s_{0}\right\}\right)$ to a boundary point of $\lambda_{1}$.

Join the endpoints of $\gamma^{\prime}$ and $\lambda_{1}$ in $f^{-1}\left(\partial^{\prime} C \cap\left\{h \geq s_{0}\right\}\right)$ and call the resulting curve $\lambda$. Orient $\lambda$ so that the part which coincides with $\gamma^{\prime}$ is correctly oriented. Then the integral of $-d \phi$ over $\lambda$ has the form $m \cdot 2 \pi$ for some integer $m$. 
Note that if $m \leq 0$, then it follows (using Lemma 9.3) that the integral of $-d \phi$ over a disjoint union of $\operatorname{arcs}$ in $\gamma\left(s_{0}\right)$ is at least $3 \pi / 4$ (assuming that $s$ is small) and this implies Lemma 9.6. Thus, one can assume that $m$ is positive.

Now, remark that using standard Morse theory, a basis for $H_{1}\left(D_{0} ; \mathbb{Z}\right)$ can be constructed so that each basis element is a continuously embedded loop in $D_{0}$ which is a union of embedded arcs. Here, each arc lies in $f^{-1}\left(\gamma\left(s_{0}\right)\right)=$ $\left(f^{*} h\right)^{-1}\left(s_{0}\right)$ or in a level set of $f^{*} \phi$. Since the integer $m$ is positive, there is at least one such generator on which $-f^{*} d \phi$ integrates to a positive multiple of $2 \pi$. This also implies Lemma 9.6.

Step 14 Now consider the case where $f^{*} h$ has some degenerate critical points and/or where the level sets of $f^{*} \phi$ are not in general position. In either case, one can perturb $f^{*} h$ and the pseudo-gradient vector field in small disks about the critical points of $f^{*} h$ so that the resulting function, $h^{\prime}$, has non-degenerate critical points; and so that the resulting pseudo-gradient flow lines are in general position.

In this regard, note that these perturbations can be assumed to be supported in a union, $V \subset D_{0}$, of disks of arbitrarily small (but positive) radius about the critical points of $h$. This means, in particular, that the new function, $h^{\prime}$, can be assumed to agree with $\mathrm{h}$ except in $V$. And, the new gradient flow lines are level sets of $f^{*} \phi$ except in $V$ and except (possibly) near $f^{-1}\left(\partial^{\prime} C\right)$. Furthermore, since $f^{*} h$ is a harmonic function on $C_{0}$ (with respect to an appropriate metric), the function $h^{\prime}$ can be assumed to have only index 1 critical points also. Thus, the number of such critical points can be taken to be independent of the diameter of the disks which comprise $V$.

(The point here is that there is some complex coordinate for $C_{0}$ near each critical point of $f^{*} h$ for which the latter is the real part of a holomorphic function on a small disk in $\mathbb{C}$. One can then consider standard perturbations of such functions.)

With the preceding understood, standard Morse theory arguments can be used to construct the 1-skeleton of $D_{0}$ as follows: Start with the $\operatorname{arcs}$ of $f^{-1}\left(\gamma\left(s_{0}\right)\right)=$ $\left(f^{*} h\right)^{-1}\left(s_{0}\right)$ and add additional arcs with end points on those of $\left(f^{*} h\right)^{-1}\left(s_{0}\right)$. The latter arcs lie in level sets of $f^{*} \phi$ except where they intersect $V$. Furthermore, the number of such arcs can be assumed independent of the diameter of $V$, and the length in $V$ of each such arc can be assumed bounded by a uniform multiple of the diameter of $V$. (Remember that $f^{*} h$ is the real part of a holomorphic function with respect to some complex coordinate on a neighborhood of each critical point.) 
Given this 1-skeleton, repeat the arguments of the previous step. The only difference here comes from the fact that $-f^{*} d \phi$ may have non-zero integral over an arc segment in $V$. However, the contribution from the segments in $V$ can be made negligibly small in absolute value because of the following facts:

- Each arc segment in $V$ has length bounded by a multiple of the radius of a disk in $V$.

- $f^{*} d h=0$ at the center of each disk component of $V$, so $f^{*} d \phi=0$ there as well. Thus, $\left|f^{*} d \phi\right|$ is small in $V$ when the disks of $V$ have small radii.

Acknowledgement The author is supported in part by the National Science Foundation.

\section{References}

[1] N Aronszajn, A unique continuation theorem for elliptic differential equations or inequalities of the second order, J. Math. Pures Appl. 36 (1957) 235-249

[2] S K Donaldson, An application of gauge theory to the topology of 4-manifolds, J. Diff. Geom. 18 (1983) 279-315

[3] S K Donaldson, Connections, cohomology and the intersection forms of 4manifolds, J. Diff. Geom. 24 (1986) 275-341

[4] MH Freedman, The topology of four dimensional manifolds, J. Diff. Geom. 17 (1982) 357-453

[5] M Gromov, Pseudo-holomorphic curves in symplectic manifolds, Invent. Math. 82 (1985) 307-347

[6] H Hofer, K Wysocki, E Zehnder, Properties of pseudo-holomorphic curves in symplectisations, I: Asymptotics, Analyse Nonlinear 13 (1996) 337-379

[7] H Hofer, K Wysocki, E Zehnder, Properties of pseudo-holomorphic curves in symplectisations, IV: Asymptotics with degeneracies, from: "Contact and Symplectic Geometry", C Thomas, editor, Cambridge University Press (1996)

[8] H Hofer, M Kreiner, Holomorphic curves in contact dynamics, to appear

[9] K Honda, Harmonic forms for generic metrics, preprint (1997)

[10] K Honda, Local properties of self-dual harmonic 2-forms on a 4-manifold, preprint (1997)

[11] C LeBrun, Yamabe constants and the perturbed Seiberg-Witten equations, preprint (1997)

[12] C Luttinger, unpublished 
[13] D McDuff, Singularities and positivity of intersections of J-holomorphic curves, with Appendix by Gang Liu, from: "Proceedings of CIMPA Summer School of Symplectic Topology", Nice 1992, Birkhauser (1994)

[14] D McDuff, D Salamon, J-Holomorphic curves and quantum cohomology, American Mathematical Society, Providence (1996)

[15] C B Morrey, Multiple Integrals in the Calculus of Variations, Springer, New York (1996)

[16] P Pansu, Pseudo-holomorphic curves in symplectic manifolds, from: "Holomorphic Curves in Symplectic Geometry", M. Audin and F. Lafontaine, editors, Progress in Math 117, Birkhauser (1994) 233-250

[17] T Parker, J Wolfson, Pseudo-holomorphic maps and bubble trees, Journ. Geom. Anal. 3 (1993) 63-98

[18] C H Taubes, The geometry of the Seiberg-Witten invariants, from: "Surveys in Differential Geometry", 1996, S T Yau, editor, International Press, to appear

[19] C H Taubes, Self-dual connections on 4-manifolds with indefinite intersection matrix, J. Diff. Geom. 19 (1984) 517-560

[20] CH Taubes, $S W \Rightarrow G r$ : From the Seiberg-Witten equations to pseudoholomorhic curves, Jour. Amer. Math. Soc. 9 (1996) 845-918

[21] CH Taubes, $S W \Rightarrow G r$ : From the Seiberg-Witten equations to pseudoholomorphic curves, from: " $S W=G r$ : The Equivalence of the Seiberg-Witten and Gromov Invariants", R Stern, editor, International Press, to appear

[22] CH Taubes, Seiberg-Witten invariants and pseudoholomorphic subvarieties for self-dual, harmonic 2-forms, preprint (1998)

[23] C H Taubes, The geometry of the Seiberg-Witten invariants, from: "Proceedings of the International Congress of Mathematicians, Berlin, 1998", Vol II, Documenta Mathematica (1998) 493-506

[24] R Ye, Gromov's compactness theorem for pseudo-holomorphic curves, Trans. Amer. Math. Soc. 342 (1994) 671-694 


\section{Appendix The proof of Assertion 3(a) of Proposition 7.1}

The proof is broken into three parts, where each part is further decomposed into separate steps. Part I of this Appendix discusses in some detail the behavior of $C$ in a small neighborhood of one of its $u>0$ singular points. Part II uses the analysis from the first part of the Appendix to prove Assertion 3(a) of Proposition 7.1 under a restrictive assumption. The final part of this Appendix proves that the restrictive assumption in part II is always valid.

Before starting, a digression is in order to set the stage. In particular, attention is focused on a neighborhood in $S^{1} \times B^{3}$ of a point $t_{0} \in Z=S^{1} \times\{0\}$. By assumption $C$ is a finite energy, pseudo-holomorphic curve and $t_{0}$ is a regular point for $C$ where $q_{ \pm}=0$. Thus, $|z| / \rho<<1$ on $C$ near $t_{0}$.

\section{Part I The local model where $u>0$}

This part of the Appendix constitutes a digression of sorts to present a local model for $C$ in the neighborhood of $u>0$ singular point. This part of the proof is broken into six steps.

Step 1 To begin, consider a regular point $t_{0} \in Z$. As in subsection 8.a, introduce the $(t, u)$ coordinate system on a neighborhood of $t_{0}$ as above so that $t_{0}=(0,0)$. For $\delta>0$ and small, let $A=\{(t, u): t \in[-\delta, \delta]$ and $u \in[0, \delta]\}$. According to the discussion in subsection 8.a, when $\delta$ is small, $C$ (in a neighborhood of $t_{0}$ ) can be described by a multivalued graph $\Phi=\left\{\left(\phi_{j}, \nu_{j}\right)\right\}_{1 \leq j \leq p}$ over $A$, where each $\left(\phi_{j}, \nu_{j}\right)$ is a locally defined function on the complement of a set $\pi(\Lambda)$, in the interior of $A$. Here $\Lambda \subset C$ is a certain set with at most countably many elements and no accumulation points, while $\pi$ denotes the projection induced map from the range of $\Phi$ in $C$ to $A$. (The set $\Lambda$ consists of the singular points of $C$ and the critical points of the function $t$.) To conserve notation, henceforth use $C$ to denote only the image of $\Phi$.

According to Lemma 8.3, the map $\Phi$, as a map into the $p^{\text {th }}$ symmetric product of $\mathbb{R}^{2}$, is Holder continuous with some exponent $\alpha>0$. In addition, given $\varepsilon>0$, one can choose $\delta$ so that each $\left(\phi_{j}, \nu_{j}\right)$ obeys (8.2) and so that (8.3) holds on the complement of $\Lambda$ in the interior of $A$. One can also assume (8.4).

Note that $\left\{\left(\phi_{j}, \nu_{j}\right)\right\}$ are obtained by restricting the functions $(\phi, \nu)$ on $S^{1} \times B^{3}$ to $C$. Further progress requires a digression to introduce a replacement, $b(\nu)$, for the function $\nu$ on the subset of $S^{1} \times B^{3}$ where $z / \rho<1 / 32$. Here is $b$ :

$$
b(\nu)=\int_{0}^{\nu}\left(1+6 s^{2}\right)^{-\frac{1}{2}}\left(1+2 s^{2}\right)^{-1} d s=\nu+\mathcal{O}\left(\nu^{3}\right) .
$$

Note that $b$ is an analytic function of $\nu$ where the latter is less than $1 / 32$.

Geometry $\&$ Topology, Volume 2 (1998) 
The advantage of replacing $\nu$ with $b$ arises when (8.3) is written in terms of the complex variables $w=t+i \cdot u$ and $\eta=\phi+i \cdot b$. The resulting equation has the schematic form

$$
\bar{\partial} \eta+\alpha_{1} \partial \eta-\frac{3}{2} u^{-1}\left(1+\alpha_{2}\right) b=0
$$

where $b=\operatorname{im}(\eta)$ while $\alpha_{1}$ and $\alpha_{2}$ are real valued, real analytic functions of $b^{2}$ where $|b| \leq 1$. Furthermore, both are $\mathcal{O}\left(b^{2}\right)$ near $b=0$. (The analogous equation for $\eta^{\prime}=\phi+i \cdot \nu$ has a term with derivatives on $\bar{\eta}^{\prime}$. The presence of such a term complicates the already complicated discussion to come.)

To derive (A.2), consider $\nu$ as a function of $b$ instead of vice-versa. Then the $b-$ derivative of $\nu$ obeys $\nu^{\prime}=\left(1+\kappa_{1}\right)^{1 / 2}\left(1+\kappa_{2}\right)^{1 / 2}$, where $\kappa_{1}$ and $\kappa_{2}$ appear in (8.3). With this understood, one finds (A.2) with $\alpha_{1}=(u-g) /(u+g)$ and $\alpha_{2}=u^{3} \rho^{-2}(u+g)^{-1}-1$. Note that in terms of $w$ and $\eta$, the sheets of $C$ over $A-\pi(\Lambda)$ are described by a set $\left\{\eta_{j}\right\}_{1 \leq j \leq p}$ of locally defined solutions of (A.2). Alternately, $\left\{\eta_{j}\right\}$ can be thought of as a Holder continuous map from $A$ into $\operatorname{Sym}^{p}(\mathbb{C})$.

Step 2 Introduce now the smooth model $C_{0}$ as described in Definition 1.1. Restricting to a subset of $C_{0}$ gives a smooth, complex curve $C_{A}$ with a proper, pseudo-holmorphic map $f$ from $C_{A}$ into $S^{1} \times\left(B^{3}-\{0\}\right)$ whose image is $\pi^{-1}(A-\{u=0\})$. By assumption, $f$ is $1-1$ on the complement of a countable subset. Note that the composition $\pi \cdot f$ is generally not a holomorphic map $f$ from $C_{A}$ into $A$. However, this map is close to being holomorphic where $u$ is small. Indeed, let $v$ be a local, complex coordinate for $C_{A}$. Then, $f_{*} \partial_{v}=t_{v} \partial_{t}+u_{v} \partial_{u}+h_{v} \partial_{h}+\phi_{v} \partial_{\phi}$ and

$$
J \cdot f_{*} \partial_{v}=-t_{v} g u^{-1} \partial_{u}+u_{v} u g^{-1} \partial_{t}+h_{v}\left(g \rho^{2}\right)^{-1} \partial_{\phi}-\phi_{v} g \rho^{2} \partial_{h} .
$$

The condition that $f$ is $J$-pseudo-holmorphic requires $J \cdot f_{*} \partial_{v}=-i \cdot \partial_{v}$; and the latter requires (in part) that $t_{v}-i \cdot u_{v}(u / g)=0$. The complex conjugate of this last equation can be rewritten as

$$
\bar{\partial}_{v} w-\alpha_{1} \bar{\partial}_{v} \bar{w}=0
$$

where $\alpha_{1}=(u-g) /(u+g)$. Since $\alpha_{1}$ is $\mathcal{O}\left(b^{2}\right)=\mathcal{O}\left(u^{2}\right)$, this last equation implies that $\pi \cdot f$ is nearly holomorphic where $u$ is small.

By the way, this last equation with (A.2) implies that that $\eta$ as a function of $v$ obeys

$$
\bar{\partial} \eta-\frac{3}{2}\left(\bar{\partial}_{v} \bar{w}\right) u^{-1}\left(1+\alpha_{2}\right) b=0 .
$$

Now suppose that $v$ is a complex parameter for some disk $D \subset C_{A}$ and that $f$ maps the origin (where $v=0$ ) to a point with coordinates $w=w_{0}$ and $\eta=\eta_{0}$. Of course (A.4) implies that $w$ is a real analytic function of $v$ (when $|v|$ is small). In particular, this last fact with (A.4) implies that the parameter $v$ can be chosen so that

$$
w(v, \bar{v})=w_{0}+v^{k}+\alpha_{1}\left(b_{0}\right) \bar{v}^{k}+\mathcal{R}(v) .
$$

Here $k \in\{1, \ldots p\}$ and $\mathcal{R}$ is a real analytic function of $v$ which obeys $|\mathcal{R}| \leq \zeta \cdot|v|^{k+1}$. Note that the upper bound of $p$ here stems from the following two facts: First, $f$ is 
almost everywhere 1-1 onto $C$. Second, the projection $\pi$ from $C$ to $A$ has at most $p$ sheets.

In the case where $w_{0} \notin \pi(\Lambda)$, then $f^{*} \pi^{*} d w \neq 0$ at $v=0$, and this implies that $k=1$ is the only possibility. However, when $w_{0} \in \pi(\Lambda)$, then $k>1$ is allowed. Even so, when $k=1$, then (A.6) implies that $f$ embeds a neighborhood of $v=0$ into $S^{1} \times\left(B^{3}-\{0\}\right)$. In this case, there is a disk $U$ surrounding $w_{0}$ with the property that $\left.C\right|_{U}$ has at least two irreducible components, and at least one of these is an embedded disk. (An irreducible component of $C$ over an open set $U \subset A$ is the closure in $\left.C\right|_{U}$ of a connected component of $\left.C\right|_{U}-\left.\Lambda\right|_{U}$.)

Step 3 Once again, let $\pi: C \rightarrow A$ denote the projection induced map and use $\pi$ to define the pull-back space $\pi^{*} C$. The latter consists of the space of triples $\left(w, \eta, \eta^{\prime}\right)$ where $w \in A$ and where both $\eta$ and $\eta^{\prime}$ are points in $\pi^{-1}(w)$. This space $\pi^{*} C$ is a manifold at points $\left(w, \eta, \eta^{\prime}\right)$ where neither pair $(w, \eta)$ nor $\left(w, \eta^{\prime}\right)$ is a singular point of $C$. In particular $\pi^{*} C$ is smooth near $\left(w, \eta, \eta^{\prime}\right)$ when $w \notin \pi(\Lambda)$. The space $\pi^{*} C$ has two canonical projections to $C$ which are denoted by $\pi_{ \pm}$below. Here $\pi_{-}$sends $\left(w, \eta, \eta^{\prime}\right)$ to $(w, \eta)$ while the image of this point under $\pi_{+}$is $\left(w, \eta^{\prime}\right)$. Moreover, note that $C$ embeds canonically in $\pi^{*} C$ as $\{(w, \eta, \eta)\}$, that is, where $\pi_{+}=\pi_{-}$.

The remainder of this step describes the irreducible components of a neighborhood of the point $\left(w_{0}, \eta_{0}, \eta_{0}^{\prime}\right)$ in $\pi^{*} C$. (Remember: an irreducible component of a neighborhood $\mathcal{U} \subset \pi^{*} C$ of $\left(w_{0}, \eta_{0}, \eta_{0}^{\prime}\right)$ is defined to be the closure in $\mathcal{U}$ of a component of $\left.\mathcal{U}\left(w_{0}, \eta_{0}, \eta_{0}^{\prime}\right).\right)$

The simplest case to consider is when $w_{0} \notin \pi(\Lambda)$. Here there is a disk $U \subset A$ centered about $w_{0}$ which has empty intersection with $\pi(\Lambda)$. Then $\left.C\right|_{U}$ is the disjoint union of $p$ copies of $U$, and then $\pi^{*} C$ over $\left.C\right|_{U}$ is the disjoint union of $p^{2}$ copies of $U$, where $p^{2}-p$ copies lie in $\pi^{*} C-C$. In particular, $\left.\pi^{*} C\right|_{U}$ near $\left(w_{0}, \eta_{0}, \eta_{0}^{\prime}\right)$ is diffeomorphic to $U$ via the obvious projection.

The structure of $\pi^{*} C$ near $\left(w_{0}, \eta_{0}, \eta_{0}^{\prime}\right)$ is more complicated than $w_{0} \in \pi(\Lambda)$. In this case it proves useful to consider separately the cases where $\eta_{0} \neq \eta_{0}^{\prime}$ and $\eta_{0}=\eta_{0}^{\prime}$. In the first case, $\left(w_{0}, \eta_{0}\right)$ and $\left(w_{0}, \eta_{0}^{\prime}\right)$ lie in distinct components of $\left.C\right|_{U}$, and then a neighborhood of $\left(w_{0}, \eta_{0}, \eta_{0}^{\prime}\right)$ over $U$ is homeomorphic to the fiber product of these two components. In particular, if the first component intersects $\pi^{-1}\left(U-w_{0}\right)$ as a $k$-sheeted cover of $U-w_{0}$, and if the second component likewise defines a $k^{\prime}$-sheeted cover, then there is a neighborhood of $\left(w_{0}, \eta_{0}, \eta_{0}^{\prime}\right)$ in $\pi^{*} C$ which intersects $\pi^{-1}\left(U-w_{0}\right)$ as a $k \cdot k^{\prime}$ sheeted cover of $U-w_{0}$. Furthermore, the number of irreducible components of $\pi^{*} C$ near $\left(w_{0}, \eta_{0}, \eta_{0}^{\prime}\right)$ is equal to the greatest common divisor of $k$ and $k^{\prime}$.

Note that the preceding assertions follow from (A.6) in as much as the latter implies that when $\left|w-w_{0}\right|$ is small but not zero, there are precisely $k$ distinct points in the $v$-disk which map to $w$. Furthermore, each such point is nearly a $k^{t h}$ root of $w-w_{0}$. In fact, according to (A.6), each $v$ which maps to the given $w$ differs from a $k^{\text {th }}$ root of $w-w_{0}$ (and vice versa) by $\mathcal{O}(\varepsilon)$ where $\varepsilon=\left|w-w_{0}\right|^{1 / k}\left(|u|^{2}|+| w-\left.w_{0}\right|^{1 / k}\right.$ ).

The second case to consider has $\eta_{0}=\eta_{0}^{\prime}$. In this case $\pi^{*} C-C$ intersects a neighborhood of $\left(w_{0}, \eta_{0}, \eta_{0}\right)$ in $\left.\pi^{*} C\right|_{U}$ in components which can be described as follows: The 
components are partially labeled by ordered pairs of irreducible components of $\left.C\right|_{U}$. This label for a point $\left(w, \eta, \eta^{\prime}\right)$ corresponds to the ordered pair of irreducible components which respectively contain $(w, \eta)$ and $\left(w, \eta^{\prime}\right)$. With the preceding understood, let $B$ be an irreducible component of $\left.C\right|_{U}$ whose intersection with $\pi^{-1}\left(U-w_{0}\right)$ defines a $k$-sheeted cover of $U-w_{0}$. Let $B^{\prime}$ be a distinct irreducible component of $\left.C\right|_{U}$ and define $k^{\prime}$ from $B^{\prime}$ in an analogous manner. Then the pair $\left(B, B^{\prime}\right)$ label a certain number of irreducible components of some neighborhood in $\pi^{*} C$ of the given point $\left(w_{0}, \eta_{0}, \eta_{0}\right)$. And this number is (as in the first case) equal to the greatest common divisor of $k$ and $k^{\prime}$.

On the other hand, if $B=B^{\prime}$, then the pair $(B, B)$ labels $k-1$ distinct components of the intersection of $\pi^{*} C$, and thus $k$ irreducible components of some neighborhood $\left(w_{0}, \eta_{0}, \eta_{0}\right)$ in $\pi^{*} C$. (Precisely one of these components lies in $C$.)

Note that the preceding assertions also follow from (A.6).

Step 4 The preceding description of $\pi^{*} C$ can be made somewhat more explicit with the help of a certain parametrization of $\pi^{*} C$ near points in $\Lambda$. Consider first the parametrization for $\pi^{*} C$ near a point $\left(w_{0}, \eta_{0}, \eta_{0}^{\prime}\right)$ where $w_{0} \in \pi(\Lambda)$. For this purpose, fix respective, irreducible components $B$ and $B^{\prime}$ for $C$ near $\left(w_{0}, \eta_{0}\right)$ and for $\left(w_{0}, \eta_{0}^{\prime}\right)$ as in Step 3. Let $a$ denote the greatest common divisor of $k$ and $k^{\prime}$, and write $k=\lambda \cdot a$ and $k^{\prime}=\lambda^{\prime} \cdot a$, where $\lambda$ and $\lambda^{\prime}$ are relatively prime.

As remarked in Step 3, a component of $\pi^{*} C$ near $\left(w_{0}, \eta_{0}, \eta_{0}^{\prime}\right)$ is labeled by an integer $s \in\{0, \ldots, a-1\}$ in addition to the label by the pair $\left(B, B^{\prime}\right)$. A coordinate for this component is given by a complex parameter $\tau$ for a small radius disk in $\mathbb{C}$ centered at 0 . The following lemma describes the parametrization of points in $\pi^{*} C$ by $\tau$.

Lemma A.1 There is a constant $\zeta \geq 1$ with the following significance: Suppose that $w_{0} \in \pi(\Lambda)$ is a point with $u_{0} \leq \zeta^{-1}$. Let $\left(w_{0}, \eta_{0}, \eta_{0}^{\prime}\right) \in \pi^{*} C$ be given, and let $\left(B, B^{\prime}\right)$ and $s$ parametrize an irreducible component of a neighborhood of $\left(w_{0}, \eta_{0}, \eta_{0}^{\prime}\right)$ in $\pi^{*} C$ as described above. Write a neighborhood of $\left(w_{0}, \eta_{0}\right)$ in $B$ as the image via the pseudo-holomorphic map $f$ of a disk $D \subset C_{0}$ with coordinate $v$ as in (A.6), and likewise parametrize a neighborhood of $\left(w_{0}, \eta_{0}^{\prime}\right)$ in $B^{\prime}$ by the $f$ image of a disk $D^{\prime}$ with complex coordinate $v^{\prime}$. Then a neighborhood of the point $\left(w_{0}, \eta_{0}, \eta_{0}^{\prime}\right)$ in the given component of $\pi^{*} C$ is parametrized by a complex coordinate $\tau$ on a disk about the origin in $\mathbb{C}$ by writing

$$
\begin{aligned}
v & =\tau^{\lambda^{\prime}} \\
v^{\prime} & =\exp \left(2 \pi i s / k^{\prime}\right) \tau^{\lambda}(1+m(\tau))
\end{aligned}
$$

where $m$ is a certain function of $\tau$ which is smooth where $\tau \neq 0$, Lipschitz near $\tau=0$ and satisfies the bound $|m| \leq \zeta\left(|\tau|^{\lambda}+|\tau|^{\lambda^{\prime}}\right)$.

Proof of Lemma (A.1) The function $m(\tau)$ is chosen so that $w(v(\tau))=w\left(v^{\prime}(\tau)\right)$. In particular, if (A.6) is used for both $v$ and $v^{\prime}$ then this last condition can be written as follows

$$
\begin{aligned}
& v^{k}\left[(1+m)^{k^{\prime}}-1\right]+\alpha_{1}\left(b_{0}\right) \bar{v}^{k}\left[(1+\bar{m})^{k^{\prime}}-1\right]= \\
& \mathcal{R}\left(\tau^{\lambda^{\prime}}\right)-\mathcal{R}\left(\exp \left(2 \pi i s / k^{\prime}\right) \tau^{\lambda}(1+m(\tau))\right) .
\end{aligned}
$$


This algebraic equation for $m$ can be written in fixed point form as

$$
m=v^{-k} k^{\prime-1}\left(\mathcal{R}\left(\tau^{\lambda^{\prime}}\right)-\mathcal{R}\left(e^{2 \pi i s / k^{\prime}} \tau^{\lambda}\right)\right)-\alpha_{1}\left(b_{0}\right) \bar{v}^{k} / v^{k} \bar{m}+\mathcal{K},
$$

where $\mathcal{K}$ is an analytic function which satisfies $|\mathcal{K}| \leq \zeta\left(u|m|^{2}+|\tau|^{\lambda}|m|\right)$.

The appropriate solution to (A.9) will be found with the help of the contraction mapping theorem. For this purpose, it proves useful to first replace $\tau$ in (A.11) by an indeterminant $x_{1}$ and to likewise replace $v /|v|$ by an indeterminant $x_{2}$. Then (A.11) has the schematic form

$$
m=\mathcal{F}\left(m, x_{1}, x_{2}\right),
$$

where $\mathcal{F}$ is a real analytic function of its entries when $u_{0}$ is small, when $m$ and $x_{1}$ take values in a small radius disk in $\mathbb{C}$, and when $x_{2}$ take values in a disk of radius 2 . Furthermore,

$$
\begin{aligned}
|\mathcal{F}| & \leq \zeta\left(\left|x_{1}\right|^{\min \left(\lambda, \lambda^{\prime}\right)}+u_{0}|m|\right) \\
\left|\partial_{m} \mathcal{F}\right| & \leq \zeta\left(\left|x_{1}\right|^{\lambda}+u_{0}\right)
\end{aligned}
$$

where $u_{0},\left|x_{1}\right|$ and $|m|$ are all smaller than some fixed constant $\zeta^{-1}$.

Given (A.11), the contraction mapping theorem finds $\zeta \geq 1$ such that when $u_{0}$ and $\left|x_{1}\right|$ are both less than $\zeta^{-1}$, and $\left|x_{2}\right| \leq 2$, then (A.10) has a unique, small norm solution $m=m\left(x_{1}, x_{2}\right)$. In addition, the contraction mapping theorem guarantees that this solution obeys $|m| \leq \zeta \cdot\left|x_{1}\right|^{\min \left(\lambda, \lambda^{\prime}\right)}$ and varies with $x_{1}$ and $x_{2}$ as a real analytic function. With this last point understood, then $m$ in Lemma A.1 is obtained from this fixed point $m\left(x_{1}, x_{2}\right)$ of $(\mathrm{A} .10)$ by setting $x_{1}=\tau$ and $x_{2}=v /|v|$.

Step 5 The set $\left\{\eta_{j}\right\}$ is the push-forward via the map $\pi$ of a well-defined function on $C$, namely the complex function $\eta=\phi+i \cdot b$. Likewise, the set of differences $\left\{\eta_{j}-\eta_{k}\right\}$ is the push-forward from $\pi^{*} C$ of the complex function $\Delta \equiv \pi_{-}^{*} \eta-\pi_{+}^{*} \eta$ via the map $\pi$ from $\pi^{*} C$ to $A$. In particular, this function $\Delta$ maps $\pi^{*} C-C$ to $\mathbb{C}-\{0\}$. Of interest in this step is the behavior of $\Delta$ on the neighborhoods in $\pi^{*} C$ which are described in Lemma A.1. For this purpose it proves convenient to use (A.5) to obtain an equation for $\pi^{*} C$ for $\Delta$. Consider:

Lemma A.2 There exists $\zeta \geq 1$ with the following significance: Suppose that $w_{0} \in$ $\pi(\Lambda)$ is a point with $u_{0}<\zeta^{-1}$. Let $\left(w_{0}, \eta_{0}, \eta_{0}^{\prime}\right) \in \pi^{*} C$ be given, and let $\left(B, B^{\prime}\right)$ and $s$ parametrize an irreducible component of a neighborhood of $\left(w_{0}, \eta_{0}, \eta_{0}^{\prime}\right)$ in $\pi^{*} C$ as described above. As in Lemma (A.1), let $\tau$ parametrize a neighborhood of $\left(w_{0}, \eta_{0}, \eta_{0}^{\prime}\right)$ in the given irreducible component. Then the function $\Delta$ pulls back as a function of $\tau$ to obey an equation of the form

$$
\bar{\partial}_{\tau} \Delta+\mathcal{J} \cdot \operatorname{im}(\Delta)=0
$$

where $\mathcal{J}$ is a function of $\tau$ which is smooth except at $\tau=0$, but is, in any event, bounded near $\tau=0$. In fact,

$$
|\mathcal{J}| \leq \zeta^{\prime}\left(u_{0}\right)|\tau|^{\lambda^{\prime}-1},
$$


where $\zeta^{\prime}$ depends, as indicated, on $u_{0}$.

Proof of Lemma A.2 To obtain (A.12), first subtract the $\eta=\eta_{k}$ version of (A.2) from the $\eta=\eta_{j}$ version, pull the result up to $\pi^{*} C$, and then pull back to the $\tau$ disk to obtain an equation for $\Delta$. The latter has the following schematic form:

$$
\begin{gathered}
\bar{\tau}_{\bar{w}} \bar{\partial}_{\tau} \Delta+\tau_{\bar{w}} \partial_{\tau} \Delta+\alpha_{1-}\left(\bar{\tau}_{w} \bar{\partial}_{\tau} \Delta+\tau_{w} \partial_{\tau} \Delta\right)+\left(\alpha_{1+}-\alpha_{1-}\right)\left(\bar{\tau}_{w} \bar{\partial}_{\tau} \eta_{+}+\tau_{w} \partial_{\tau} \eta_{+}\right) \\
-\frac{3}{2} u^{-1}\left(b_{-}-b_{+}+\left(\alpha_{2} b_{-}\right)-\left(\alpha_{2} b_{+}\right)\right)=0
\end{gathered}
$$

Here $b_{ \pm}=\operatorname{im}\left(\eta_{ \pm}\right), \alpha_{1 \pm} \equiv \alpha_{1}\left(b_{ \pm}\right)$and $\alpha_{2 \pm} \equiv \alpha_{2}\left(b_{ \pm}\right)$. Meanwhile, $\tau_{w} \equiv \partial_{w} \tau, \quad \bar{\tau}_{w}=$ $\partial_{w} \bar{\tau}$, etc. To simplify this last equation, remark first that (A.4) and the first point of (A.7) imply that $\tau_{\bar{w}}=-\alpha_{1-} \tau_{w}$. Thus the terms with $\partial_{\tau} \Delta$ in (A.14) cancel. Then using Taylor's theorem with remainder, (A.14) can be rewritten as

$$
\bar{\tau}_{\bar{w}}\left(1-\left|\alpha_{1-}\right|^{2}\right) \bar{\partial}_{\tau} \Delta+\mathcal{J}^{\prime} \operatorname{im}(\Delta)=0
$$

where $\left|\mathcal{J}^{\prime}\right| \leq \zeta\left(u_{0}\left(1+\sup _{j}\left|\partial_{w} \eta_{j}\right|\right)+u_{0}^{-1}\right)$. In fact, because $\eta$ pulls back to $C_{A}$ as a smooth function of $v$, while $w$ obeys (A.6), it follows that $\sup _{j}\left|\partial_{w} \eta_{j}\right| \leq \zeta\left|w-w_{0}\right|^{-1+1 / k}$ near $w_{0}$. This last bound implies that $\left|\mathcal{J}^{\prime}\right| \leq \zeta|\tau|^{-\lambda \lambda^{\prime} a+\lambda^{\prime}}$ since $u_{0}$ is bounded away from zero near $w_{0}$. Meanwhile, it follows from (A.4) and the first point of (A.7) that $\bar{\tau}_{\bar{w}}\left(1-\left|\alpha_{1-}\right|^{2}\right)=1 / w_{\tau}$, so (A.15) implies (A.12) with $\mathcal{J}=w_{\tau} \mathcal{J}^{\prime}$, and so (A.6) and (A.7) give $|\mathcal{J}| \leq \zeta|\tau|^{\lambda^{\prime}-1}$ as claimed.

Step 6 Equations (A.12) and (A.13) can now be used to analyze the behavior of $\Delta$ as a function of the parameter $\tau$ from Lemmas A.1 and A.2. The results are summarized by:

Lemma A.3 Continue with the assumptions of Lemma A.2. The constant $\zeta$ in said lemma can be chosen so that the following additional conclusion holds: Suppose that $\tau$ does not parametrize a subset of $C$ in $\pi^{*} C$. Where the parameter $\tau$ obeys $|\tau| \leq \zeta^{-1}$, the function $\Delta$ can be written as

$$
\Delta=\operatorname{re}\left(\eta_{0}-\eta_{0}^{\prime}\right)+e^{\kappa} \Delta_{0}
$$

where $\Delta_{0}$ is a non-zero, holomorphic function of $\tau$ with $\operatorname{re}\left(\Delta_{0}(0)\right)=0$. Also, the function $\kappa$ is continuous, and smooth except possibly at $\tau=0$; in any event, $|\nabla \kappa| \leq$ $\zeta|\ln (\tau)|$. In addition, if $\operatorname{im}\left(\eta_{0}-\eta_{0}^{\prime}\right)=0$, then

$$
\Delta_{0}=c_{n} \tau^{n}+\mathcal{O}\left(|\tau|^{n+1}\right),
$$

where $n \geq 1$ is an integer, and where $c_{n}$ is a nonzero constant. Furthermore, in this last case, $\left|\nabla^{j} \kappa\right|$ is bounded for all $j \in\left\{0, \ldots, \lambda^{\prime}-1\right\}$ and vanishes at the origin.

Proof of Lemma A.3 Let $\underline{\Delta} \equiv \Delta-\operatorname{Re}\left(\eta_{0}-\eta_{0}^{\prime}\right)$. Note that im $\underline{\Delta}=\operatorname{im}(\Delta)$. With this understood, choose $\kappa$ to obey the equation $\partial_{\tau} \kappa+\mathcal{J} \cdot \operatorname{im} \underline{\Delta} / \underline{\underline{\Delta}}=0$ near the origin of the $\tau$ plane. This and (A.12) imply that $\Delta_{0}$ is holomorphic and $\operatorname{Re}\left(\Delta_{0}(0)\right)=0$. In this regard, note that the condition $\Delta_{0} \equiv 0$ implies that $\pi_{-} \eta-\pi_{+} \eta$ is constant near $\tau=0$. 
Since $\Delta$ is real analytic on $\pi^{*} C-C$ away from $\pi^{-1}(\Lambda)$, then $\pi_{-} \eta-\pi_{+} \eta$ is constant on the whole of a component of $\pi^{*} C$, and this is forbidden by the given assumptions. As $\Delta_{0}$ is not identically zero, then $\Delta_{0}$ cannot be constant when $\operatorname{im}\left(\eta_{0}-\eta_{0}^{\prime}\right)$ vanishes. This implies the assertion about (A.17).

Now consider the assertions about the derivatives of $\kappa$. To begin remark that the equation in the preceding paragraph for $\kappa$ has infinitely many solutions since any holomorphic function can be added to any one solution. Nonetheless, there is at least one solution with the required behavior, as the following argument shows: Extend the differential equation for $\kappa$ to the whole of the $\tau$ plane by replacing $\mathcal{J}$ with $\chi_{r} \mathcal{J}$, where $\chi_{r}(\tau) \equiv \chi(|\tau| / r)$ with $\chi$ the standard bump function and $r>0$ a small, positive number. The solution for $\kappa$ is $\kappa(\tau)=\pi^{-1} \int\left(\tau^{\prime}-\tau\right)^{-1} \chi_{r} \mathcal{J} \operatorname{im} \underline{\Delta} / \underline{\Delta} d^{2} \tau^{\prime}+\kappa_{0}$, where $\kappa_{0}$ is a polynomial function of the complex variable $\tau$. Here $\kappa_{0}$ is chosen so that $\kappa$ and its derivates to order $\lambda^{\prime}-1$ vanish at the origin. In this regard (A.16) is invoked to estimate the derivatives to order $\lambda^{\prime}-1$ of the function $\pi^{-1} \int\left(\tau^{\prime}-\tau\right)^{-1} \chi_{r} \mathcal{J i m} \underline{\Delta} / \underline{\Delta} d^{2} \tau^{\prime}$ at $\tau=0$.

Note that the procedure just outlined fails to produce the asserted bound for $|\nabla \kappa|$ only in the case when $\lambda^{\prime}=1$. The argument for this case rests on the following claim: The function $\mathcal{J} \operatorname{im} \Delta / \Delta$ can be written as $\nu(\tau, \tau /|\tau|)$, where $\nu(\cdot, \cdot)$ is a smooth function on $\mathbb{C}^{2}$. Granted this claim, differentiate the integral expression for $\kappa$ given in the previous paragraph to find that as long as $\tau \neq 0$, one has $|\nabla \kappa| \leq \zeta \int_{\left|\tau^{\prime}-\tau\right| \leq 2 r}\left|\tau^{\prime}-\tau\right|^{-1}\left|\tau^{\prime}\right|^{-1} d^{2} \tau^{\prime}$. The integral here is bounded by $\zeta|\ln (\tau)|$ which is the required bound.

Argue as follows for the claim in the preceding paragraph: First, say that a function of $\tau$ is two-variable smooth if it can be written as $\nu(\tau, \tau /|\tau|)$, where $\nu$ is a smooth function on $\mathbb{C}^{2}$. A basic fact to be used below is the following: if a function $\sigma$ is 2-variable smooth, and $|\tau|^{-1} \sigma$ is bounded near $\tau=0$, then $|\tau|^{-1} \sigma$ is also two variable smooth. (Use Taylor's theorem to write $\nu=\tau \nu_{+}+\bar{\tau} \nu_{-}$, where $\nu_{ \pm}$are two-variable smooth.)

With the preceding understood, note that $\Delta$ will fail as a smooth function of $\tau$ only when $v^{\prime}$ in (A.7) does not have smooth dependence on $\tau$; and the latter can happen due to the presence of the function $m(\cdot)$. However, it follows from (A.10) that $m$ is two-variable smooth. However, because $\partial \Delta$ is bounded, so it must also be twovariable smooth. This implies the claim directly in the case where $\Delta_{0}(0) \neq 0$. When $\Delta_{0}(0)=0$, then according to (A.17), one has $\Delta_{0}=\tau^{n} \iota(\tau)$, where $\iota$ is complex analytic and non-vanishing at 0 . Thus $\triangleq$ has the form $\tau^{n} \sigma$ where $\sigma$ is 2 -variable smooth and non-vanishing at $\tau=0$. In this case, $\partial \underline{\Delta}=|\tau|^{-1} \tau^{n} \sigma_{1}$, where $\sigma_{1}$ is 2-variable smooth. This last observation implies that $\mathcal{J} \operatorname{im}(\underline{\Delta}) / \underline{\Delta}$ (which equals $\underline{\Delta}^{-1} \bar{\partial} \underline{\underline{\Delta}}$ ) has the form $|\tau|^{-1} \sigma_{2}$ where $\sigma_{2}$ is $2-$ variable smooth. However, $\mathcal{J} \operatorname{im}(\underline{\Delta}) / \underline{\Delta}$ is bounded, so it must also be 2 -variable smooth.

\section{Part II A finiteness proposition for $\Lambda$ under a density assump- tion}

The main result here, Proposition A.7, proves Assertion 3(a) of Proposition 7.1 under an auxiliary assumption whose justification is deferred to Part III of this Appendix. 
This portion of the proof of Assertion 3(a) of Proposition 7.1 is broken into ten steps.

Step 1 To begin, consider a regular point $t_{0} \in Z$ and reintroduce $\delta>0$, the set $A$ and the coordinates $w=t+i \cdot u$ and $\eta=\phi+i \cdot b$ as in Step 1 of Part I. According to Lemma 8.3, the assignment of $w$ to $\left\{\eta_{j}(w)\right\}=\pi^{-1}(w) \subset C$ defines a continuous map from $A$ to $\operatorname{Sym}^{p} \mathbb{C}$, and the continuity of this map facilitates a stratification of a neighborhood of the point $t_{0}$ as $\bigcup_{1<n<p} A_{n}$, where a point $w \in A_{n}$ if $n$ or more elements from $\left\{\eta_{j}(w)\right\}$ coincide. Note that each $A_{n}$ is closed; and that $A_{n}-A_{n+1}$ is relatively open in $A_{n}$. Thus each $w \in A-A_{n+1}$ has an open neighborhood which is also in $A-A_{n+1}$. (This all follows from Lemma 8.3.) Furthermore, without loss of generality, one can assume that $A_{2}$ consists of the union of $\pi(\Lambda)$ with some subset of $Z$.

With $A_{n}$ understood, let $Z_{n} \equiv A_{n} \cap Z$.

This stratification of $Z$ will now be used to formulate an induction proof that $\Lambda$ is finite. Here is how: Suppose that $n \geq 2$ and that for all $m<n$, it has been established that all limit points of $A_{m}$ are in $Z_{n}$. The induction step will prove that all limit points of $\bigcup_{m \leq n} A_{m}$ lie in $Z_{n+1}$. For this purpose it is sufficient to assume that the given point $t_{0}$ lies in $Z_{n}$ and then prove that $t_{0}$ is an isolated point of $A_{n}$. With this understood, remark that $\delta$ is chosen small in Step 1 of Part I, then $A \cap A_{n+1}=\emptyset$ and this implies that it is sufficient to consider only the case $n=p$ in the induction step.

Here is one last remark for this step: By taking $\delta$ smaller if necessary, one can arrange, with no generality lost, that each $\phi_{j}$ takes values only in $(-1 / 4,1 / 4)$ on $A$.

Step 2 This step introduces the following basic assumption:

The set $Z-Z_{p}$ is dense in $Z$ near $t_{0}$.

The justification of this assumption is deferred to Part III of this Appendix.

The assumption in (A.18) is used in the subsequent arguments solely to deduce the existence of points $t_{+} \in(0, \delta / 100]$ and $t_{-} \in[-\delta / 100,0)$ which lie in $Z$ where all $\left\{\phi_{j}\right\}$ are distinct. Since the set of such points is open and since $\Lambda$ has at most countably many elements, there is no obstruction to choosing $t_{ \pm}$and then $s_{0}>0$ so that the line segments where $t=t_{ \pm}, u \in\left[0, s_{0}\right]$ miss all points of $\pi(\Lambda)$ and such that the set of functions $\left\{b_{j}\right\}$ are distinct where $t=t_{ \pm}$and $u \in\left(0, s_{0}\right]$.

Step 3 Now introduce the function $\Delta$ on $\left.\pi^{*} C\right|_{A}$ as in Step 5 of Part I, and denote the real part of $\Delta$ by $\phi$ and the imaginary part by $\underline{b}$. This step considers the behavior of $d \underline{\phi}$ where $\underline{b}=0$. The next lemma summarizes the results.

The statement of the lemma introduces the notion of an embedded graph $\Gamma$ in a manifold. For the present purposes this term is defined as follows: Call the manifold $X$. Then $\Gamma$ is a locally compact subset of $X$ with certain additional properties. First, $\Gamma$ has a distinguished subset $\Gamma_{v}$ which is a locally finite set of points. (Each point $\Gamma_{v}$ is called a vertex.) Second, $\Gamma-\Gamma_{v}$ is a locally finite collection of properly embedded, open intervals in $X-\Gamma_{v}$. (The closure of each component of $\Gamma-\Gamma_{v}$ in $X$ is called 
an edge.) Third, each vertex of $\Gamma$ has a neighborhood in $X$ whose intersection with $\Gamma$ consists of a finite union of properly embedded images of $[0,1)$ by an embedding which sends 0 to the vertex in question. Moreover, these embedded intervals intersect pairwise only at the given vertex.

Lemma A.4 Let $\Gamma \subset \pi^{*} C-\left(C \cup \pi_{+}^{-1}(\Lambda) \cup \pi_{-}^{-1}(\Lambda)\right)$ denote the zero set of the function $\underline{b}$. Then $\Gamma$ is an embedded graph with the following properties.

- The vertices of $\Gamma$ are the $\underline{b}=0$ critical points of $\underline{b}$ and there are at most finitely many of these in any subset of $\pi^{*} C-\left(C \cup \pi_{+}^{-1}(\Lambda) \cup \pi_{-}^{-1}(\Lambda)\right)$ which has compact closure.

- The one form $d \underline{\underline{\phi}}$ restricts without zeros to each edge of $\Gamma$.

- At each point of $\Gamma-\Gamma_{v}$, the two form $d \underline{\phi} \wedge d \underline{b}$ orients $\pi^{*} C$ so that $\pi$ is an orientation preserving map to $A$.

- There are an even number and more than two edges of $\Gamma$ ending in each critical point of $\underline{b}$. These edges are oriented so that $d \phi$ increases moving towards the vertex on precisely half of these edges.

Proof of Lemma A.4 The fact that $\Gamma$ is a graph whose vertices are the critical points of $d \underline{b}$ follows from the assertion that $d \underline{b}$ defines a real analytic function on the complement in $\pi^{*} C-C$ of $\pi_{+}^{-1}(\Lambda) \cup \pi_{-}^{-1}(\Lambda)$. To prove this claim, consider a small radius disk $D \subset A$ where $u>0$ which avoids all points of $\pi(\Lambda)$. Then $\left.C\right|_{D}$ consists of $p$ disjoint copies of $D$, and each $\eta \in\left\{\eta_{j}\right\}$ is a bonafide function on $D$ which obeys (A.2). It then follows from this last point with standard elliptic regularity results (as in Chapters 5 and 6 of [15]) that each such $\eta$ defines a real analytic function on $D$.

Meanwhile, $\left.\pi^{*} C\right|_{D}$ consists of $p^{2}$ copies of $D$ with $p^{2}-p$ copies being disjoint from $C$. With the preceding understood, let $D^{\prime} \subset \pi^{*} C-C$ denote one of the latter copies of $D$. Then $w$ pulls back via $\pi$ as a bonafide coordinate of $D^{\prime}$ and then the analyticity of each $\eta_{j}$ as a function of $w$ implies that $\Delta$ is also an analytic function of $w$.

To see that $\underline{b}$ has at most finitely many critical points on $D^{\prime}$, observe that there will be an infinite number of such critical points only if $\underline{b}=$ constant on $D^{\prime}$. Thus $\underline{b}=$ constant on the component of $\pi^{*} C$ which contains $D^{\prime}$. And this last possibility is ruled out by the assumptions in Step 1.

The analysis of the behavior of $d \phi$ on $\Gamma$ requires the observation that $\Delta$ on $D^{\prime}$ obeys an equation having the schematic form

$$
\bar{\partial} \Delta+\alpha_{1-} \cdot \partial \Delta+\mathcal{R} \cdot \underline{b}=0
$$

where $\alpha_{1-} \equiv \alpha_{1}\left(\pi_{-}^{*} b\right)$ and where $\mathcal{R}$ is a real analytic function on $D^{\prime}$. In particular, at points of $\Gamma$, the complex function $\Delta$ obeys the equation $\bar{\partial} \Delta+\alpha_{1} \cdot \partial \Delta=0$. Since $\left|\alpha_{1}\right| \leq \zeta \cdot u^{2}$, it follows that where $u$ is small, $|\bar{\partial} \Delta|^{2}<<|\partial \Delta|^{2}$, which implies both the second and third points of Lemma A.4.

The final point of (A.19) can be deduced using (A.19) and the analyticity of $\Delta$ to write down a local model for $\Delta$ near a critical point where $\underline{b}=0$. Indeed, it follows from (A.19) that near such a point, $\Delta=\underline{\phi}_{0}+c \cdot\left(w^{m}-\alpha_{1}\left(b_{0}\right) \cdot \bar{w}^{m}\right)+\mathcal{O}\left(|w|^{m+1}\right)$ for 
some $m>1$. (Here $\underline{\phi}_{0}$ is the value of $\underline{\phi}$ at the critical point, and $b_{0}$ is the value of $\pi_{-}(b)$ at the critical point.) It then follows from this last expression that the condition $\operatorname{im}(\Delta)=0$ defines exactly $2 m$ embedded line segments emanating from the critical point, these being approximately the $2 m$ rays where the $w^{m}$ is real. The question of the orientations is left to the reader to verify from this local expression for $\Delta$.

Step 4 This step considers the behavior pf $\Gamma$ near the points of the form $\left(w_{0}, \eta_{0}, \eta_{0}^{\prime}\right)$ where $w_{0} \in \pi(\Lambda)$ and $\eta_{0} \neq \eta_{0}^{\prime}$. The first observation in this regard is that $\Gamma$ will miss this singular point of $\pi^{*} C$ unless the imaginary parts of $\eta_{0}$ and $\eta_{0}^{\prime}$ agree, so suppose that this is the case. Introduce from Lemma A.1 the complex parameter $\tau$ on an irreducible component of a neighborhood of $\left(w_{0}, \eta_{0}, \eta_{0}^{\prime}\right)$ in $\pi^{*} C$. It follows from (A.17) and the bound in Lemma A.3 on $|d \kappa|$ by $\zeta|\ln (\tau)|$ that there are no $\underline{b}=0$ critical points of $\underline{b}$ in some small neighborhood of $\left(w_{0}, \eta_{0}, \eta_{0}^{\prime}\right)$. These same points from Lemma A.3 also imply that there is some non-zero, and even number of edges of $\Gamma$ whose closure contains $\left(w_{0}, \eta_{0}, \eta_{0}^{\prime}\right)$, and that half of these point away from $\left(w_{0}, \eta_{0}, \eta_{0}^{\prime}\right)$ with the $d \underline{\phi}>0$ orientation, while half point towards $\left(w_{0}, \eta_{0}, \eta_{0}^{\prime}\right)$ with this orientation.

Step 5 This step considers the behavior of $\Gamma$ near points $\left(w_{0}, \eta_{0}, \eta_{0}^{\prime}\right)$ with $w_{0} \in \pi(\Lambda)$. For this purpose, let $\tau$ be the local parameter from Lemma A.1 on an irreducible component of a neighborhood of $\left(w_{0}, \eta_{0}, \eta_{0}^{\prime}\right)$ in $\pi^{*} C$. Suppose, in addition, that this irreducible component does not lie in $C$. Once again, let $r>0$ be small and let $\gamma$ denote the circle where $|\tau|=r$. And, for almost all $r$, the circle $\gamma$ will intersect $\Gamma$ transversely. (In fact, it follows from (A.17) and that bound on $|d \kappa|$ in Lemma A.3 that $\gamma$ and $\Gamma$ are transverse whenever $r$ is sufficiently small.)

Now let $\kappa, \kappa^{\prime}, \quad \lambda, \lambda^{\prime}$ and $a$ be as in Lemma A.3. Then it follows from Lemma A.3 and (A.17) that there are as many segments of $\Gamma$ which intersect $\gamma$ and point outward with the $d \underline{\phi}>0$ positive orientation as point inward. Furthermore, there is at least one such segment pointing in each direction with the $d \underline{\phi}>0$ orientation.

Step 6 This step extends the graph $\Gamma$ to allow for vertices and edges on the $u=0$ line. For this purpose it is necessary to first digress to consider the behavior of $\Gamma$ near points in the $u=0$ line which do not lie in $Z_{p}$. Let $t_{1}$ be such a point. It follows from the induction hypothesis that $C$ is smooth near any such point and thus over some semi-disk neighborhood $D^{\prime}=\left\{(t, u): u \geq 0\right.$ and $\left.u^{2}+\left(t-t_{1}\right)^{2}<r^{2}\right\}$ of such a point, $C$ consists of some $p$ sheets, with each being diffeomorphic to $D^{\prime}$ via the projection $\pi$. In particular, the coordinates $(t, u)$ give coordinates on each such sheet. More to the point, over $D^{\prime}$, the functions $\left\{\eta_{1}, \ldots, \eta_{p}\right\}$ are distinguishable and each is analytic. Furthermore, according to Assertion 3(c) of Proposition 7.1, each such $\eta_{j}$ has the form $\eta_{j}=\phi_{0 j}(t)+i \cdot 4^{-1} \phi_{0 j}^{\prime}(t) \cdot u+\mathcal{O}\left(|u|^{2}\right)$ where $\phi_{0 j}(t)$ is an analytic function of $t$ near $t_{1}$. This last observation implies that $\Delta$ restricts to a component of $\left.\pi^{*} C\right|_{D^{\prime}}$ to have the form $\Delta=\underline{\phi}_{0}(t)-8^{-1} \underline{\phi}_{0}^{\prime \prime} \cdot u^{2}+i \cdot 4^{-1} \underline{\phi}_{0}^{\prime}(t) \cdot+\mathcal{O}\left(u^{3}\right)$, where $\underline{\phi}_{0}$ is an analytic function of $t$ near $t_{1}$. Note that $d \underline{\phi} \wedge d \underline{b}=4^{-1}\left|\underline{\phi}_{0}^{\prime}\right|^{2} d t \wedge d u+\mathcal{O}(u)$ and thus even where $u=0$, this form gives the same orientation for $\pi^{*} C$ as does $d t \wedge d u$.

With the preceding understood, agree to extend the graph $\Gamma$ to the portion of the closure $\left.\pi^{*} C\right|_{A}$ which lies over the complement of $Z_{p}$ in the $u=0$ line. The extra 
vertices are the points over the complement of $Z_{p}$ in the $u=0$ line where $\phi_{0}^{\prime}=0$. The interiors of the extra edges are the component intervals of the set of points where $u=0$ both $t \neq 0$ and also $\underline{\phi}_{0}^{\prime} \neq 0$.

Step 7 This step digresses to consider the behavior of $\Gamma$ near one of these new vertices. In the simplest case, $\phi_{0}$ has a non-degenerate critical point over $t_{1} \notin Z_{p}$ on the $u=0$ line. Near such a critical point, Assertion 3(c) of Proposition 7.1 has

$$
\Delta=c \cdot\left[\left(t-t_{1}\right)^{2}-4^{-1} \cdot u^{2}+i \cdot 4^{-1}\left(t-t_{1}\right) \cdot u\right]+\mathcal{O}\left(u^{3}\right)
$$

with $c \neq 0$. Thus $\Gamma$ has three edges which end at the vertex $\left(t_{1}, 0\right)$, these being the segments where

- $u=0$ and $t<t_{1}$

- $u=0$ and $t>t_{1}$

- $u>0$ and $t=t_{1}$ (to leading order in $u$ ).

The $d \phi$ orientations for the latter are equivalent to that given, respectively, by $\operatorname{sign}(c \bar{d} t, \operatorname{sign}(c) d t$ and $-\operatorname{sign}(c) d u$. Thus the positive $d \underline{\phi}$ orientation for the first two segments make both point either towards or away from $t_{1}^{-}$together, and then the third segment points opposite, away or towards $t_{1}$, respectively.

The following lemma states the situation in general.

Lemma A.5 Fix a vertex of $\Gamma$ over the $u=0$ line. This vertex lies over a point $t_{1} \notin Z_{p}$ and is a critical point of $\phi_{0}$. If the order of vanishing of $d \phi_{0}$ at $t_{1}$ is even, then there are at least four and an even number of edges of $\Gamma$ which are incident to this vertex. If the order of vanishing is odd, there are at least three and an odd number of edges of $\Gamma$ which are incident to this vertex. In either case, two of these project to the $u=0$ line, one to where $t<t_{1}$ and the other to where $t>t_{1}$. The remaining edges lie, except for their vertices, where $u>0$. Furthermore, for $r>0$ but small, each such edge intersects the half circle where $\left(t-t_{1}\right)^{2}+u^{2}=r^{2}$ and $u \geq 0$ transversely in exactly one point. By following this half circle from its start at $u=0$ and $t=t_{1}-r$ to its end at $u=0$ and $t=t_{1}+r$, the edges are met consecutively, and consecutive edges point in opposite directions (either towards or away from $t_{1}$ ) when oriented to have $d \phi_{0}>0$. (If the order of vanishing of $d \underline{\phi}_{0}$ at $t_{1}$ is $N$, then it can be proved that there are exactly $N+2$ edges of $\Gamma$ which are incident on the vertex $t_{1}$.)

Proof of Lemma A.5 Introduce polar coordinates $(r, \theta) \in[0,1) \times[0, \pi]$ near $t_{1}$ by writing $t-t_{1}=r \cos \theta$ and $u=r \sin \theta$. Then, as $\Delta$ is analytic near $t_{1}$, it can be written as

$$
\Delta=\underline{\phi}_{0}\left(t_{1}\right)+r^{N+1} \cdot(f(\theta)+i \cdot g(\theta))+\mathcal{O}\left(r^{N+2}\right) .
$$

Here, $f$ and $g$ are real analytic functions on $[0, \pi]$ which, because of (A.2), are constrained to obey

$$
\begin{array}{ll}
\text { - } & \sin ^{-3} \theta\left(\sin ^{3} \theta f_{\theta}\right)_{\theta}+(N+1)(N+4) f=0 \\
\text { - } & f_{\theta}(0)=f_{\theta}(\pi)=0 \\
\text { - } g=f_{\theta} /(N+4) .
\end{array}
$$


Because $f$ is constrained here to be an analytic function on $[0, \pi]$, the first line in (A.23) implies the following:

- The coefficients in a power series solution for $f$ about any point are determined by the value of $f$ and its derivative at that point.

In particular, this last point implies that there is a unique (up to multiplication by real numbers) solution to the first two constraints (A.23). The fact that there is a nontrivial solution is not obvious, but such is indeed the case. It is left to the reader to verify that the first two points in (A.23) are satisfied by a polynomial of order $(N+1)$ in $\cos \theta$ which is either even or odd (depending on the parity of $N+1$ ) under the involution $\theta \rightarrow \pi-\theta$.

Note that (A.24) also precludes both $f_{\theta \theta}$ and $f_{\theta}$ vanishing simultaneously. This implies that the zeros of $g$ are non-degenerate. Then (A.22) implies that for small, but positive $r$, the graph $\Gamma$ has no vertices save that at $t_{1}$, and it implies that each edge of $\Gamma$ which is incident on this vertex has exactly one intersection point with the half circle of constant $r$, and that the intersection there is transverse.

Here is an argument for the equality between the parity of the number of zeros of $f_{0}$ and the parity of the order of vanishing of $d \underline{\phi}_{0}$ at $t_{1}$ : Consider the case where the parity of the latter is odd (the even case is argued along similar lines). Odd order of vanishing of $d \phi_{0}$ implies that $f(0)=f(\pi)$. On the other hand, the analyticity of $f$ and the first two points of (A.23) imply that $f_{\theta \theta}$ and $f$ have opposite signs at both 0 and $\pi$. Thus, $f_{\theta \theta}(0)=f_{\theta \theta}(\pi)$ also. In as much as $f_{\theta}$ has transverse zeros, this last condition implies that $f_{\theta}$ must have an odd number of zeros in the interval $(0, \pi)$.

The following is an argument for the existence of at least three zeros of $f_{\theta}$ (counting those at $\theta=0$ and $\theta=\pi$ ): When $f$ and $f^{\prime}$ are solutions to the first two lines of (A.23) which correspond to different values of $N$, then integration by parts shows that $f_{\theta}$ and $f_{\theta}^{\prime}$ are orthogonal with respect to the measure $\sin ^{3} \theta d \theta$ on $[0, \pi]$. As the $N=0$ solution to the first two lines of (A.23) is $\cos \theta$, this orthogonality implies that each $N>0$ solution $f$ obeys $\int f_{\theta} \sin ^{4} \theta d \theta=0$. Hence $f_{\theta}$ must change sign on the interval $[0, \pi]$ and so it has at least three zeros.

Step 8 This step serves as a digression to introduce the family of maps $\psi$ from either $[0,1]$ or $[0,1)$ or $(0,1]$ into $A \times \mathbb{C} \times \mathbb{C}$ with the following properties:

- $\operatorname{Im}(\psi) \subset \operatorname{closure}(\Gamma)$.

- $\psi$ is $1-1$ onto its image.

- Let $e \subset \Gamma$ be the interior of an edge. Then $\psi^{-1}(e)$ is either empty or connected, and in the latter case, $\psi$ restricts to $\psi^{-1}(e)$ as a smooth, orientation preserving embedding. Here, the orientation on $e$ is defined so that $d \underline{\phi}>0$.

A map $\psi$ which obeys the constraints in (A.25) will be called an oriented, graphical path.

Step 9 To begin the discussion here, introduce $t_{ \pm}$and $s_{0}$ from Step 2. If $s_{0}$ is sufficiently generic, then $\pi(\Lambda)$ has empty intersection with the line segment with $t \in$ $\left[t_{-}, t_{+}\right]$and where $u=s_{0}$. Furthermore, if $s_{0}$ is generic, then $\Gamma$ has no vertices which 
project to the $u=s_{0}$ line segment, nor does it have non-transversal intersections with the inverse image of this segment. Thus, with $s_{0}$ generic, the subset of $\Gamma$ where $u \leq s_{0}$ intersects the level set $u=s_{0}$ in some finite number, say $N$ points, Also by Step 2's assumption, $t \in\left(t_{-}, t_{+}\right)$on $\Gamma$.

The first claim is:

Lemma A.6 Assume that $t_{0}$ is an isolated point of $Z_{p}$. If $N>0$, then there exists a set $\left\{\psi_{j}\right\}$ of no more than $N$ and at least $N / 2$ oriented graphical paths in $A \times \mathbb{C} \times \mathbb{C}$ with the following properties:

- The map $\psi_{j}$ sends any endpoints of its domain to the $u=s_{0}$ line.

- If the domain of $\psi_{j}$ is $[0,1)$ or $(0,1]$, then the closure of the image of $\psi_{j}$ consists of the union of the image of $\psi_{j}$ with a non-empty set of points which map by $\pi$ to $Z_{p}$.

- If the image of $\psi_{j}$ intersects a vertex where $t=t_{1}$ on the $u=0$ line, then precisely one of these possibilities occurs:

a) $\psi_{j}$ both enters and exits this vertex along edges where $u>0$.

b) $\psi_{j}$ both enters and exits this vertex along edges where $u \equiv 0$.

c) $\psi_{j}$ enters along the $u=0$ edge where $|t|>\left|t_{1}\right|$ and it exits an edge where $u>0$.

d) $\quad \psi_{j}$ enters along an edge where $u>0$ and exits along the $u=0$ edge where $|t|<\left|t_{1}\right|$.

- The image of $\psi_{j}$ intersects at most one $\Delta=0$ point of $\pi^{-1}(\Lambda)$. And, if such a point is in the image of $\psi_{j}$, then the domain of $\psi_{j}$ is $[0,1]$.

- Every point of $\Gamma$ on the $u=s_{0}$ line is in the image of some $\psi_{j}$.

- When $j \neq i$, then the image of $\psi_{j}$ intersects that of $\psi_{k}$, if at all, only at vertices or at points of $\pi^{-1}(\Lambda)$.

The remainder of this step is occupied with the proof of Lemma A.6.

Proof of Lemma A.6 The maps $\left\{\psi_{j}\right\}$ will be constructed consecutively starting with $\psi_{1}$. To construct $\psi_{1}$, introduce the subgraph $\Gamma_{1} \subset \Gamma$ which is obtained from the latter by deleting the following subset of $u \equiv 0$ edges:

- An edge where $t>0$ is deleted if the $d \underline{\phi}>0$ orientation agrees with the $d t>0$ orientation.

- An edge where $t<0$ is deleted if the $d \underline{\phi}>0$ orientation disagrees with the $d t>0$ orientation.

Note that $\Gamma$ and $\Gamma_{1}$ agree where $u>0$.

The graph $\Gamma_{1}$ is an example of a subgraph $\Gamma^{\prime} \subset \Gamma$ with the following properties:

- Every vertex of $\Gamma^{\prime}$ intersects an even number of edges, half of which are incoming and half of which are outgoing when oriented so that $d \phi>0$. If a vertex is on the $u=0$ line, say with $t$ value equal to $t_{1}$, then an incident $u \equiv 0$ edge is outgoing if and only if $|t| \leq\left|t_{1}\right|$ on this edge. 
- Every point in $\pi_{+}^{-1}(\Lambda) \cup \pi^{-1}(\Lambda)$ intersects the closure in $A \times \mathbb{C} \times \mathbb{C}$ of an even number of edges of $\Gamma^{\prime}$. Furthermore, half of these are incoming and half are outgoing when they are oriented so that $d \underline{\phi}>0$.

- The closure of $\Gamma^{\prime}$ in $A \times \mathbb{C} \times \mathbb{C}$ is the union of the subgraph, a set of points which map by $\pi$ to $Z_{p}$, and a subset of $\pi^{-1}(\Lambda)$.

- The subgraph $\Gamma^{\prime}$ has non-empty intersection with the $u=s_{0}$ line.

(The first point here follows from Lemmas A.4 and A.5, and the second point from the discussions in Steps 4 and 5.)

Let $\Gamma^{\prime} \subset \Gamma$ be a subgraph which obeys the points of (A.27). The subsequent construction in this step produces an oriented, graphical path $\psi$ which satisfies the first four points in Lemma A.6 and which lies in the closure of $\Gamma^{\prime}$. To begin, select a point where $\Gamma^{\prime}$ intersects that $u=s_{0}$ line segment and follow the corresponding edge of $\Gamma^{\prime}$ to where $u<s_{0}$. This begins to trace out the image of graphical path $\psi:\left[0, \delta^{\prime}\right) \rightarrow \Gamma^{\prime}$ for some $\delta^{\prime}>0$ which is either orientation preserving or reversing, depending on whether the edge is oriented inward or outward along the $u=s_{0}$ segment. For the sake of argument, suppose that this $\psi$ is orientation preserving. (If not, reparametrize the interval $[0,1]$ by inverting about $1 / 2$.) The map $\psi$ can be extended indefinitely by walking down the given edge unless one of the following occur:

- $\quad$ The $u=s_{0}$ segment is intersected.

- A $u>0$ vertex of $\Gamma^{\prime}$ is reached.

- $\quad$ The image of $\pi \cdot \psi$ limits to a point in $\pi_{+}^{-1}(\Lambda) \cup \pi_{-}^{-1}(\Lambda)$.

- $\quad$ A $u=0$ vertex of $\Gamma^{\prime}$ is reached.

If the first case in (A.28) occurs, then stop because up to reparametrization of the domain of definition, a graphical path $\psi$ obeying the first four points of Lemma A.6 has been constructed.

Assume that the second point of (A.28) occurs. In this case, the image of $\psi$ enters the vertex on an inward oriented edge, and therefore the map $\psi$ can be extended to a larger domain so that the extension leaves the given vertex on one of the outward oriented edges. (Use the first point of (A.27) here.) This extends $\psi$ as an oriented, graphical path.

Likewise, if the third point of (A.28) occurs, then $\psi$ can also be extended as an oriented, graphical path. This follows from the second point of (A.27).

If the fourth point of (A.28) occurs, let $t_{1}$ denote the $t$ coordinate of this vertex. Note that $\psi$ entered this vertex from an edge which has $u>0$ on its interior. If there is an outgoing edge with $u>0$ on its interior, then $\psi$ can be extended by continuing along this end. If not, then there is an outgoing edge with $u \equiv 0$ and that one has $|t|<\left|t_{1}\right|$. (Use the second point in (A.27).) Extend $\psi$ by continuing along this last outgoing edge.

Now one can continue to extend $\psi$ by walking along the interior of the edge just chosen until one of the possibilities in (A.28) reoccurs. If the first point occurs, then stop and the resulting $\psi$ is an oriented, graphical path in the closure of $\Gamma^{\prime}$ which obeys the first four points of Lemma A.6. If the second or third points in (A.28) occur, extend $\psi$ as 
before. If the last point in (A.28) occurs, then $\psi$ can also be extended as described previously except in the case where the incoming edge has $u=0$, as this case was not previously considered. Note that in this case, the entering edge has $|t|>\left|t_{1}\right|$ due to the chosen direction for leaving a $u=0$ vertex. In any case, if there is an outgoing edge from this vertex which has $u>0$ on its interior, then extend $\psi$ by preceding along this vertex. If not, then the other $u=0$ edge (which has $|t|<\left|t_{1}\right|$ ) is outgoing, and one can extend $\psi$ by proceeding along the latter.

These last steps can be iterated repeatedly to extend $\psi$ as an oriented, graphical path indefinitely unless point one in (A.28) occurs. In this case, $\psi$ is an oriented, graphical path in the closure of $\Gamma^{\prime}$ which obeys the first four points of Lemma A.6. (Note that the image of $\psi$ can intersect at most one $\Delta=0$ point in $\pi_{+}^{-1}(\Lambda) \cup \pi_{-}^{-1}(\Lambda)$ because $\phi=\operatorname{Re}(\Delta)$ is zero at such points but is increasing along the image of $\psi$. Also, as $\phi$

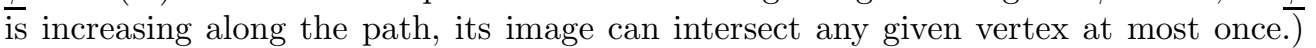
If the first point in (A.28) never occurs, then the construction just described proceeds without end to construct a map, $\psi$, from $[0,1)$ into $A \times \mathbb{C} \times \mathbb{C}$. However, in this last case, the closure of the image will be the union of the image of $\psi$ with a non-empty subset of $Z_{p}$. Thus, in this case, the first four points of Lemma A.6 are also obeyed. (In this regard, note that if the domain of $\psi$ is equal to $[0,1)$, then no $\Delta=0$ points are in the $\psi$ 's image as $\Delta=0$ at the points which project by $\pi$ to $Z_{p}$ and $\phi=\operatorname{re}(\Delta)$ is increasing along the image of $\underline{\phi}$.)

With the preceding construction understood, apply it to the case where $\Gamma^{\prime}=\Gamma_{1}$ and let $\psi_{1}$ denote the corresponding $\psi$. Now consider the construction of $\psi_{2}$ and, sequentially, $\psi_{j}$ for $j \geq 3$. The construction here is inductive. Given that $\psi_{j-1}$ for $j \geq 2$, as has been constructed, set $\Gamma_{j}$ to be the subgraph of $\Gamma_{j-1}$ which is obtained by deleting all interiors of edges which intersect the image of $\psi_{j-1}$. The construction just described of $\psi_{j-1}$ insures that $\Gamma_{j}$ obeys all the points of (A.27) except possibly the last one. If the last point is also obeyed, then repeat the recipe of the preceding paragraphs with $\Gamma^{\prime}=\Gamma_{j}$ and take $\psi_{j}$ to be the corresponding $\psi$. Since each $\psi_{j}$ maps into $\Gamma_{j}$, the final requirement for the set $\left\{\psi_{j}\right\}$ is automatically satisfied.

Step 10 Consider the following proposition:

Proposition A.7 Suppose that $Z-Z_{p}$ is dense in $Z$ near the point $t_{0}$. Then $\Lambda$ is finite.

The remainder of this step proves the preceding proposition.

Proof of Proposition A.7 Assume, to the contrary, that $\Lambda$ has an infinite number of points. This assumption will be seen to imply a contradiction to the fact that $\phi$ is increasing along any oriented, graphical path. To obtain such a contradiction, it is necessary to digress for a moment to introduce the subgraph $\Gamma^{\prime} \subset \Gamma$ which is obtained from the latter by deleting various edges and vertices. A vertex $v \in \Gamma$ is missing from $\Gamma^{\prime}$ when all edges which intersect $v$ either satisfy one of the conditions of (A.26) or else intersect the image of some $\psi_{j}$ in their interior. Meanwhile, an edge $e \in \Gamma$ is missing from $\Gamma^{\prime}$ if either of the conditions of (A.26) hold, or if the interior of said edge intersects 
the image of some $\psi_{j}$. Note that $\Gamma^{\prime} \neq \emptyset$ if $\Lambda$ is infinite because each $\psi_{j}$ intersects no more than one point in the set $\pi_{+}^{-1}(\Lambda) \cup \pi_{-}^{-1}(\Lambda)$ where $\Delta=0$, and there are infinitely many such points. Furthermore, the local picture of $\Gamma$ near a $\Delta=0$ point as given in Step 5 indicates that each such point is in the closure of a non zero and even number of edges of $\Gamma$. In particular, $\Gamma^{\prime}$ here satisfies all but the last condition in (A.27).

With the preceding understood, choose a $\Delta=0$ point which is in the closure of an edge from $\Gamma^{\prime}$. It follows from the discussion in Step 5 that there is at least one outgoing edge and one incoming edge (both with the $d \phi>0$ orientation) of $\Gamma^{\prime}$ which have the given point in their closure. Select such an outgoing edge and proceed along it starting from the given $\Delta=0$ point. Then the construction in the previous step produces a map $\psi$ from $[0,1)$ into $A \times \mathbb{C} \times \mathbb{C}$ which is a graphical embedding whose image is in the closure of $\Gamma^{\prime}$. By construction, $\psi$ sends 0 to the chosen $\Delta=0$ point. Note that the first point in (A.28) cannot occur becuase $\Gamma^{\prime}$ is disjoint from the $u=s_{0}$ line. It then follows from the construction that the closure of the image of $\psi$ is the union of the image of $\psi$ with some non-empty subset of points which project by $\pi$ to $Z_{p}$. However, this is impossible as $\Delta=0$ at any such point, while $\phi$ is already zero at $\psi(0)$ and then increases along the image of $\psi$.

\section{Part III The density of $Z-Z_{p}$ in $Z-Z_{p+1}$}

The purpose of this last section of the appendix is to prove the following.

Proposition A.8 Let $t_{0}$ be a regular point of $Z$ which lies in $Z-Z_{p+1}$. Then $Z-Z_{p}$ has non-empty intersection with any given neighborhood of $t_{0}$.

Note that this proposition justifies the assumption in (A.18).

The proof of this proposition is broken into fourteen steps.

Step 1 This step outlines the proof. To begin, reintroduce the domain $A$ and then the coordinates $w=t+i \cdot u$ and $\eta=\phi+i \cdot b$ as in Step 1 of part I. Thus $t_{0}$ corresponds to the point $w=0$. Next, introduce the data $\left\{\eta_{j}\right\}$ as in Step 1 of part I and let $\underline{\eta} \equiv p^{-1} \sum_{j} \eta_{j}$. Note that $\underline{\eta}$ is a bonafide, complex valued function on $A$. Set $\left\{\underline{\eta}_{j} \equiv \eta_{j}-\underline{\eta}\right\}$ and observe that all elements in $\left\{\underline{\eta}_{j}\right\}$ vanish at each point of $A_{p}$.

Fix $s_{0}>0$ but much less than the number $\delta$ which defines the size of the half-square $A$ and let $D \subset A$ denote the $u \geq 0$ radius $s_{0}$ half disk with center at $w=0$. For positive integer $n$, define data $\left\{\underline{\eta}_{j, n}\right\}$ on $D$ by dilating and rescaling $\left\{\underline{\eta}_{j}\right\}$ according to the rule

$$
\underline{\eta}_{j, n}(w)=\underline{\eta}_{j}\left(2^{-n} w\right) / \lambda_{n}
$$

Here $\lambda_{n}$ is a suitably chosen real number. Up to this factor $\left\{\underline{\eta}_{j, n}\right\}$ is obtained from $\left\{\underline{\eta}_{j}\right\}$ by pulling the latter back from the ball of radius $2^{-n} s_{0}$. In any event, the data $\left\{\underline{\eta}_{j, n}\right\}$ defines a continuous map from the radius $s_{0}$ half-disk about $w=0$ to $\mathrm{Sym}^{p} \mathbb{C}$. This construction in (A.29) should be done for each positive integer $n$ to obtain a sequence $\left\{\left\{\underline{\eta}_{j, n}\right\}\right\}_{n=1,2, \ldots}$. For the purposes of the next lemma, this sequence should 
be thought of as a sequence of maps into the space $\mathrm{Sym}^{p} \mathbb{C}$. And, for the purposes of the next lemma, $\operatorname{Sym}^{p} \mathbb{C}$ will be identified with $\mathbb{C}^{p}$ via the homeomorphism from the latter to $\operatorname{Sym}^{p} \mathbb{C}$ which associates the $p$ roots of a monic, $p^{\text {th }}$ order polynomial on $\mathbb{C}$ with the coefficients of its $p$ lower order terms.

With the preceding understood, here is the key property of this sequence:

Lemma A.9 The numbers $\left\{\lambda_{n}\right\}_{n=1,2, \ldots}$ in (A.29) can be chosen so as to be strictly decreasing to zero and so that the resulting sequence $\left\{\left\{\underline{\eta}_{j, n}\right\}\right\}_{n=1,2, \ldots}$ has an infinite subsequence which converges weakly in the Sobolev $L_{1}^{2}$ topology and strongly in the $C^{0}$ topology on the $u \geq 0$ radius $s_{0} / 16$ half-disk with center $w=0$ to a continuous non-trivial map into $\mathrm{Sym}^{p} \mathbb{C}$.

Let $D^{\prime}$ denote the radius $s_{0} / 16$ half-disk in the lemma. Also, let $\left\{\left\{\underline{\eta}_{j, \infty}\right\}_{1 \leq j \leq p}\right.$ denote the limit in Lemma A.9. Then let $C^{\prime} \subset D^{\prime} \times \mathbb{C}$ denote the closure of the set of points of the form $(w, \eta)$ where both $\left.\eta \in\left\{\underline{\eta}_{j, \infty}\right\}\right|_{w}$, and $\eta \neq 0$. Now use this $C^{\prime}$ to define the function $\tau$ on $D^{\prime}$ whose value at $w$ is $\tau(w) \equiv \prod_{(w, \eta) \in C^{\prime}} \eta$. Here is the key property of $\tau$ :

Lemma A.10 The function $\tau$ as defined above is not identically zero, it is continuous and Sobolev class $L_{1}^{2}$. Furthermore, it obeys an equation having the schematic form

$$
\bar{\partial} \tau+\frac{3 i}{4 u} p^{\prime}(1-\mathcal{P}) \tau=0
$$

where $p^{\prime} \in\{1, \ldots, p\}$ and where $\mathcal{P}$ is continuous almost everywhere on $D^{\prime}$ and has norm less than or equal to 1.

The preceding two lemmas are proved below starting in Step 3, so accept them for now.

The proof of Proposition A.8 proceeds from this point with a study of the function $\tau$. In particular, consider:

Lemma A.11 Let $\tau$ be a non-trivial solution to (A.30) on $D^{\prime}$ which is continuous and Sobolev class $L_{1}^{2}$. Then $\tau$ has isolated zeros where $u>0$ and $\tau \neq 0$ on a dense, open subset of the $u=0$ line near the origin.

This last lemma is proved below in Step 2.

With the previous two lemmas understood, the proof of Proposition A.8 ends with the following two remarks: First, given $\varepsilon>0$, there is, according to Lemma A.11, a point $t^{\prime}$ on the $u=0$ line with distance $\varepsilon$ or less from the origin where $\tau \neq 0$. Thus, $|\tau|>c>0$ at this point. Second, according to Lemma A.9, there is some (infinitely many) value of $n$ for which there is a $j$ with $\left|\underline{\eta}_{j, n}\right|>c /(2(1+c))$ at the point $t^{\prime}$. Thus at $2^{-n} t^{\prime}$ on the $u=0$ line, one has $\left|\underline{\eta}_{j}\right|>c \lambda_{n} /(2(1+c))$. Hence the point $2^{-n} t^{\prime}$ on the $u=0$ line is not in $A_{p}$.

Step 2 This step contains the proof of Lemma A.11. 
Proof of Lemma A.11 Write $\tau=u^{-3 p^{\prime} / 2} \lambda$. Then (A.30) implies the following equation for $\lambda$ :

$$
\bar{\partial} \lambda+\frac{3 i}{4 u} p^{\prime}(1-\mathcal{P}) \lambda=0 .
$$

This last equation can be used to analyze $\lambda$ by writing the latter as $e^{\kappa} \lambda_{0}$, where $\kappa$ is a solution to the equation

$$
\bar{\partial} \kappa=\frac{3 i}{4 u} p^{\prime} \mathcal{P} .
$$

and where $\lambda_{0}$ is holomorphic where $u>0$ on $A$. Of particular interest is the solution to (A.32) which is given by the integral expression

$$
\left.\kappa\right|_{w}=\frac{3 i}{4 \pi} p^{\prime} \int_{\mathbb{C}} \underline{\theta}\left(\left(w-w^{\prime}\right)^{-1}-\left(w-\bar{w}^{\prime}\right)^{-1}\right) u^{\prime-1} \mathcal{P} d^{2} w^{\prime} .
$$

Here $\underline{\theta}$ denotes the characteristic function of the radius $s_{0} / 16$ half disk on which $\tau$ is defined.

This last expression for $\kappa$ can be used to estimate $|\kappa|$. For this purpose, first write $\left(w^{\prime}-w\right)^{-1}-\left(\bar{w}^{\prime}-w\right)^{-1}=2 u^{\prime} /\left(\left(w^{\prime}-w\right)(\bar{w}-w)\right)$ and then take absolute values to find that

$$
|\kappa|_{w} \leq 3 p^{\prime}(2 \pi)^{-1} \int_{\mathbb{C}} \underline{\theta} 1 /\left(\left|w^{\prime}-w\right|\left|\bar{w}^{\prime}-w\right|\right) d^{2} w^{\prime} .
$$

In particular, suppose that the $t$ coordinate of $w$ is less than $s_{0} / 64$. Then it is an exercise to use (A.34) to bound

$$
|\kappa|_{(t, u)} \leq \zeta_{1}+\frac{3}{2} p^{\prime}|\ln (u)| .
$$

where $\zeta_{1}$ is independent of $t$ and $u$. With regard to the derivation of (A.35), note that the $\ln (u)$ term in (A.35) arises from that part of the integral in (A.34) where the coordiantes $t^{\prime}$ and $u^{\prime}$ of $w^{\prime}$ are constrained by $\left|t^{\prime}-t\right|^{2}+u^{\prime 2} \geq 100 u^{2}$. Indeed, this portion of the integral differs from

$$
3 p^{\prime}(2 \pi)^{-1} \int_{\substack{\delta \geq\left|w^{\prime}\right| \geq 10 u \\ 0 \leq \arg \left(w^{\prime}\right) \leq \pi}}\left|w^{\prime}\right|^{-2} d^{2} w^{\prime}
$$

by a $u$-independent constant. Likewise, the remaining portion of the integral is also bounded by a $u$-independent constant. Here, this last assertion follows from the fact that $1 /\left|\bar{w}^{\prime}-w\right| \leq 1 / u$ on $A$.

In any event, with (A.35) understood, it follows that $|\lambda| \geq \zeta^{-1} u^{3 p / 2}\left|\lambda_{0}\right|$ where $\zeta \geq 1$ is a fixed, $u$-independent constant. On the other hand, because $|\tau|=u^{-3 p / 2}|\lambda|$, it follows that

$$
|\tau| \geq \zeta^{-1}\left|\lambda_{0}\right|
$$

To end the story, note that (A.37) implies that $\tau$ can not vanish on a non-trivial line segment of the $u=0$ line near $(0,0)$. Indeed, if it did, then the holomorphic function $\lambda_{0}$ would vanish on a non-trivial line segment and thus vanish identically. Then, $\tau$ would vanish identically, which is assumed not to be the case. 
Step 3 This step and the subsequent steps contain the arguments which lead to the proof of Lemmas A.9 and A.10. This step serves to identify the number $\lambda_{n}$ in (A.29). For this last purpose, introduce, for positive and integral $n$, the half disk, $D(n)$, of radius $2^{n} s_{0}$ and center at $w=0$. Now, define $\lambda_{n}$ by the equality

$$
\lambda_{n}^{2} \equiv \int_{D(n)} \sum_{j}\left(\left|d \underline{\eta}_{j}\right|^{2}+u^{-2}\left|\operatorname{im}\left(\underline{\eta}_{j}\right)\right|^{2}\right) .
$$

Note that the sequence $\left\{\lambda_{n}\right\}$ is strictly decreasing to zero as advertised in Lemma A.9.

Step 4 Here are some key properties of $\left\{\underline{\eta}_{j, n}\right\}$ :

Lemma A.12 For each positive integer $n$, define $\left\{\underline{\eta}_{j, n}\right\}$ as in (A.29) with $\lambda_{n}$ as in (A.38). There is a constant $\zeta \geq 1$ which is independent of $n$ and is such that for all $n$ sufficiently large, the following is true:

- $1=\int_{D(0)} \sum_{j}\left(\left|d \underline{\eta}_{j, n}\right|^{2}+u^{-2}\left|\operatorname{im}\left(\underline{\eta}_{j, n}\right)\right|^{2}\right)$

- $\int_{D(4)} \sum_{j}\left(\left|d \underline{\eta}_{j, n}\right|^{2}+u^{-2}\left|\operatorname{im}\left(\underline{\eta}_{j, n}\right)\right|^{2}\right) \leq \zeta \int_{D(2)}\left|\underline{\eta}_{j, n}\right|^{2}$

- Let $s \in\left(0, s_{0} / 4\right)$ and let $D^{\prime} \subset D(0)$ denote the intersection of $D(0)$ with a disk of radius $s<s_{0} / 4$ and center in $D(2)$. Then, $\int_{D^{\prime}} \sum_{j}\left|d \underline{\eta}_{j, n}\right|^{2} \leq \zeta \cdot s^{1 / \zeta}$.

- The function $\theta_{n} \equiv\left(\sum_{j}\left|\underline{\eta}_{j, n}\right|^{2}\right)^{1 / 2}$ is bounded by $\zeta$ on $D(2)$ and on $D(2)$ satisfies a Holder estimate of the following form: $\left|\theta_{n}(w)-\theta_{n}\left(w^{\prime}\right)\right| \leq \zeta \cdot\left|w-w^{\prime}\right|^{1 / \zeta}$ when both $w$ and $w^{\prime}$ are in $D(2)$.

- Each $\underline{\eta}_{j, n}$ can be viewed as a well defined function on some neighborhood of all but countably many $u>0$ points of $D(1)$. On such a neighborhood, each $\underline{\eta}_{j, n}$ obeys an equation of the form

$$
\bar{\partial} \underline{\eta}_{j, n}-\frac{3}{2} u^{-1} i m\left(\underline{\eta}_{j}\right)+\sigma_{j, n} \cdot \partial \underline{\eta}_{j} \cdot \operatorname{im}\left(\underline{\eta}_{k, n}\right)=0
$$

where $\left|\sigma_{j, n}\right| \leq \zeta \cdot 2^{-n}$ and where $\left\{\sigma_{j, n}^{k^{\prime}}\right\}$ also tend to zero as $n$ tends to infinity, but in the following weaker sense: Let $s>0$ and let $D^{\prime} \subset D(0)$ be the intersection of $D(0)$ with a disk of radius $s$ in $A$. Then, $\int_{D^{\prime}} \sum_{j, k}\left|\sigma_{j, n}^{k}\right|^{2} \leq \zeta s^{1 / \zeta} 2^{-n / \zeta}$.

Proof of Lemma A.12 The first point of the lemma follows directly from the scaling relation. The proof of the second and third points are lengthy and given below. Meanwhile, the fourth point follows from the fifth point and the first point using Theorem 3.5.2 of [15]. And, the fifth point follows driectly from (A.2) with some straightforward algebraic manipulations and with the appropriate rescaling. (Note that (8.4) and (8.12) have been invoked to obtain the estimates for $\left\{\sigma_{j, n}\right\}$ and $\left\{\sigma_{j, n}^{k^{\prime}}\right\}$.) The fifth point of the lemma is used in the proof of the second and third points.

To prove the second point of the lemma, introduce a bump function $\chi$ which is 1 on $D(4)$, and zero on $D(0)-D(2)$ and which is non-increasing. Multiply (A.39) by $\underline{\chi}$. Write the term $\underline{\chi}^{\bar{\partial}} \underline{\eta}_{j, n}$ as $\bar{\partial}\left(\underline{\chi \eta}_{j, n}\right)-\bar{\partial} \underline{\chi \eta}_{j, n}$ and likewise write $\underline{\chi}^{\partial} \underline{\eta}_{j, n}$ in terms of 
$\partial\left(\underline{\chi \eta}_{j, n}\right)$. Put the terms with derivatives of $\underline{\chi}$ on the right hand side. Then, square the resulting equation and after an integration by parts, one obtains the inequality

$$
\begin{aligned}
\int_{D(1)} \sum_{j}\left(\left|d\left(\underline{\chi \eta}_{j, n}\right)\right|^{2}\right. & \left.\left.+u^{-2}\left|\operatorname{im}\left(\underline{\chi \eta}_{j, n}\right)\right|^{2}\right)-\zeta \int_{D(1)} \sum_{j, k}\left|\sigma_{j, n}^{k}\right|^{2} \sum_{m} \mid \operatorname{im} \underline{\chi \eta}_{m, n}\right)\left.\right|^{2} \\
& \leq \zeta \cdot s_{0}^{-2} \int_{D(1)}\left|\underline{\eta}_{j, n}\right|^{2}
\end{aligned}
$$

(There are no extraneous boundary terms in the integration by parts due to the presence of points in $\Lambda$. The argument here for the absence of such terms is the same as that given in the proof of Lemma 8.3 for the absence of similar terms in (8.14).)

The next claim is that the right most term on the left side of (A.40) obeys the bound

$$
\int_{D(1)} \sum_{j, k}\left|\sigma_{j, n}^{k}\right|^{2} \sum_{j^{\prime}}\left|\operatorname{im}\left(\underline{\chi \eta}_{j^{\prime}, n}\right)\right|^{2} \leq \zeta 2^{-n / \zeta} \int_{D(1)} \sum_{j}\left(\left|d\left(\underline{\chi \eta}_{j, n}\right)\right|^{2}\right.
$$

where $\zeta \geq 1$ is independent of $n$. Note that when $n$ is large, this last claim with (A.40) gives the second point of Lemma A.13. Meanwhile, the claim in (A.41) follows from Lemma 5.4.1 of [15] using the estimates for the integrals of $\sum_{j, k}\left|\sigma_{j, n}^{k}\right|^{2}$ over disks in $D(0)$. With regard to this appeal to Morrey's lemma, take for Morrey's domain $G$ the half disk $D(1)$ in the $w$-plane, and take Morrey's function $u$ to equal $\left(\sum_{j}\left|\operatorname{im}\left(\underline{\chi} \eta_{j, n}\right)\right|^{2}\right)^{1 / 2}$. (Note that the norm of the differential of the latter is no greater than $\left(\sum_{j}\left|d\left(\underline{\chi \eta}_{j, n}\right)\right|^{2}\right)^{1 / 2}$.)

The proof of the third point of Lemma A.12 requires a preliminary digression. To start the digression, take $s$ positive but less than $s<s_{0} / 4$. Then, let $S(s)$ denote a $u \geq 0$ half-disk with diameter $s$ and center at some point $\left(t^{\prime}, 0\right)$ on the $u=0$ line in $D(2)$. The purpose of this digression is to prove that

$$
\int_{S(s)} \sum_{j}\left(\left|d \underline{\eta}_{j, n}\right|^{2}+u^{-2}\left|\operatorname{im}\left(\underline{\eta}_{j, n}\right)\right|^{2} \leq \zeta \cdot s^{1 / \zeta}\right.
$$

where $\zeta \geq 1$ is independent of $s, n$ and the center point of the half disk.

What follows next is the proof of (A.42): Write $\underline{\varphi}_{j, n}$ for the real part of $\underline{\eta}_{j, n}$ and $\underline{b}_{j, n}$ for the imaginary part. Now, square both sides of (A.39), sum over $j$ and integrate the result over $S(s)$ to find (after an integration by parts) that

$$
\begin{aligned}
& \int_{S(s)} \sum_{j}\left(\left|d \underline{\eta}_{j, n}\right|^{2}+9 u^{-2}\left|\underline{b}_{j, n}\right|^{2}+6 u^{-1} \underline{b}_{j, n} \partial_{u} b_{j, n}-6 u^{-1} \underline{b}_{j, n} \partial_{t} \underline{\phi}_{j, n}\right) \\
& \leq-\int_{\partial S(s)} \sum_{j} \underline{b}_{j, n} d \underline{\phi}_{j, n}+\zeta \cdot\left(2^{-2 n} \int_{S(s)} \sum_{j}\left|d \underline{\eta}_{j, n}\right|^{2}+\int_{S(s)} \sum_{j, k}\left|\sigma_{j, n}^{k}\right| \cdot \sum_{j}\left|\underline{b}_{j, n}\right|^{2}\right) .
\end{aligned}
$$

As with the manipulations in Section 8 for the proof of Lemma 8.2, the points in $\Lambda$ do not contribute extraneous boundary terms to (A.43). Next, integrate by parts on the 
third term from the left on the top line in (A.43) to replace the latter by

$$
\int_{S(s)} 3 u^{-2} \sum_{j}\left|\underline{b}_{j, n}\right|^{2}+\int_{\partial S(s)} 3\left(\left(t-t^{\prime}\right)^{2}+u^{2}\right)^{-\frac{1}{2}} \sum_{j}\left|\underline{b}_{j, n}\right|^{2} .
$$

Thus, an application of the triangle inequality shows that the top line of (A.43) is no greater than

$$
\zeta^{-1} \cdot \int_{S(s)} \sum_{j}\left(\left|d \underline{\eta}_{j, n}\right|^{2}+u^{-2}\left|\underline{b}_{j, n}\right|^{2}\right) .
$$

Here, $\zeta$ is an $n$-independent constant.

Meanwhile, for all but a measure zero set of values for $s$, the left most term on the bottom line of (A.43) is no greater than

$$
\zeta \cdot s \cdot \int_{\partial S(s)} \sum_{j}\left(\left|d \underline{\eta}_{j, n}\right|^{2}+u^{-2}\left|\underline{b}_{j, n}\right|^{2}\right)
$$

Indeed, this follows directly by writing $\underline{b}_{j, n}=u \cdot\left(u^{-1} \underline{b}_{j, n}\right)$ and then completing the square. To analyze the right most term on the second line in (A.43), introduce $\theta_{n} \equiv$ $\left(\sum_{j}\left|\underline{b}_{j, n}\right|^{2}\right)^{1 / 2}$. As $\left|d \theta_{n}\right| \leq \sum_{j}\left|d \underline{\eta}_{j, n}\right|$, it follows from the estimate for $\left\{\sigma_{j, n}^{k}\right\}$ and from Lemma 5.4.1 in [15] that this term is no greater than $\zeta \cdot 2^{-n / \zeta} \int_{S(s)}\left(\left|d \underline{\eta}_{j, n}\right|^{2}+u^{-2}\left|\underline{b}_{j, n}\right|^{2}\right)$. Here, one should apply Lemma 5.4.1 of Morrey using $G$ to denote the half disk $S^{\prime}$ which is concentric with $S(s)$ but has twice the radius. Also, this lemma from Morrey is stated for a function $u$; and to apply this lemma, take $u$ to be a compactly supported function on $S^{\prime}$ which equals the given $\theta_{n}$ on $S(s)$ and whose $L_{1}^{2}$ norm is no greater than $\zeta \int_{S(s)}\left(\left|d \theta_{n}\right|^{2}+u^{-2}\left|\theta_{n}\right|^{2}\right)$ for some $s$ and $n$ independent constant $\zeta$. (Such a function can be obtained by reflecting $\theta_{n}$ through the boundary of $S(s)$ and then using a radial cut-off function.)

With all of the preceding understood, then (A.43) is seen to imply (for large $n$ ) the following inequality for almost all values of $s$ :

$$
\int_{S(s)} \sum_{j}\left(\left|d \underline{\eta}_{j, n}\right|^{2}+u^{-2}\left|\underline{b}_{j, n}\right|^{2}\right) \leq \zeta s \int_{\partial S(s)} \sum_{j}\left(\left|d \underline{\eta}_{j, n}\right|^{2}+u^{-2}\left|\underline{b}_{j, n}\right|^{2}\right) .
$$

Here, $\zeta$ is independent of $s$ and of $n$. This last equation has the form $F \leq \zeta s F^{\prime}$ for a function $F$ on $\left[0, s_{0}\right]$ which is almost everywhere differentiable; and such an equation can be integrated to find that $F(s) \leq \zeta \cdot s^{1 / \zeta} F\left(s_{0}\right)$. Finally, given the first point of the lemma, this last expression is (A.42).

End the digression.

With the estimate in (A.42) understood, turn now to the proof of the third point of Lemma A.12. For this purpose, there are two cases to consider. In the first case, the center point $w_{1}=t_{1}+i \cdot u_{1}$ for a disk satisfies $u_{1} \leq s / 4$. Here, the required estimate for the fourth point of Lemma A.13 follows directly from (A.42). 
The second case to consider has $u_{1} \geq 4 s$. Here, save for one major point and a few minor ones, the argument follows that part of the proof of Lemma 8.2 which begins in the paragraph after $(8.17)$. (These arguments from Section 8 can be borrowed when $n$ is large and so both $\left|\sigma_{j, n}\right|$ and $\left|\sigma_{j, n}^{k}\right|$ from (A.39) are small in the sense indicated in the statement of Lemma A.12.) Here are the necessary modifications: First, replace $\left(\varphi_{j}, \nu_{j}\right)$ with $\left(\underline{\varphi}_{j, n}, \underline{b}_{j, n}\right)$. Second, the $\zeta \cdot s_{1}^{2}$ term in $(8.18)$ should be replaced by

$$
\zeta \cdot\left(u_{1}^{-2} \int_{D} \sum_{j}\left|\underline{b}_{j, n}\right|^{2}+\int_{D} \sum_{j, k}\left|\sigma_{j, n}^{k}\right|^{2} \sum_{m}\left|\underline{b}_{m}\right|^{2}\right) .
$$

The claim now is that right most term in (A.48) is no greater than $\zeta s_{1}^{1 / \zeta}$ for some constant $\zeta \geq 1$ which is independent of $u_{1}, s_{1}$ and $n$. Accept this claim for the moment and then the $\zeta s_{1}^{2}$ term in (8.18) can ultimately be replaced by $\zeta s_{1}^{1 / \zeta}$. With this last replacement understood, then $(8.21)$ will be replaced by

$$
f \leq \zeta s_{1} f^{\prime}+\zeta s_{1}^{1 / \zeta}
$$

This last equation can be integrated from $s$ to $u_{1} / 4$ to find that

$$
f(s) \leq \zeta\left(\left(s / u_{1}\right)^{1 / \zeta} f\left(u_{1} / 4\right)+s^{1 / \zeta}\left|\ln \left(s / u_{1}\right)\right|+1\right) .
$$

Since (A.42) already bounds $f\left(u_{1} / 4\right)$ by $\zeta^{\prime} u_{1}^{1 / \zeta^{\prime}}$, for constant $\zeta^{\prime} \geq 1$ which is independent of $n$ and $u_{1}$, this last equation implies Lemma A.12's third point.

Thus, it remains only to establish the bound of (A.48) by $\zeta s_{1}^{1 / \zeta}$ for some constant $\zeta$ which is independent of $u_{1}, s_{1}$ and $n$. Here is the argument for such a bound on the left most term in (A.48): To begin, let $\theta \equiv\left(\sum_{j}\left|\underline{b}_{j, n}\right|^{2}\right)^{1 / 2}$. As $\theta$ vanishes on the $u=0$ line, this function is no greater than $\int_{0 \leq u^{\prime} \leq u}|d \theta| d u^{\prime}$ at a point $(t, u)$. Of course, this last expression is no greater than $u^{1 / 2}\left(\int_{0 \leq u^{\prime} \leq u}|d \theta|^{2} d u^{\prime}\right)^{1 / 2}$. And, since $|d \theta| \leq$ $\left(\sum_{j}\left|d \underline{b}_{j, n}\right|^{2}\right)^{1 / 2}$, this last expression is no greater than $u^{1 / 2}\left(\int_{0 \leq u^{\prime} \leq u} \sum_{j}\left|d \underline{b}_{j, n}\right|^{2}\right)^{1 / 2}$. Now, substitute the latter into the left most term (A.48) to bound said term by

$$
\zeta u_{1}^{-1} s_{1} \int_{S\left(2 u_{1}\right)} \sum_{j}\left|d \underline{b}_{j, n}\right|^{2} .
$$

(Remember that the disk $D$ is contained in the half disk $S\left(2 u_{1}\right)$.) Finally, as $s_{1} \leq u_{1} / 4$, one can use (A.42) to bound this last expression by the desired $\zeta s_{1}^{1 / \zeta}$.

Now consider the right most term in (A.48). Here, Lemma 5.4.1 of Morrey can be invoked once again given the estimates for $\sum_{j, k}\left|\sigma_{j, n}^{k}\right|^{2}$ in the fifth point of Lemma A.12. In this case, take the domain $G$ in the statement of Morrey's lemma to be the disk which is concentric to $D$ but has radius $u_{1} / 2$. Moreover, take the function $u$ in the statement of Morrey's lemma to equal the product of $\theta$ with a standard cut-off function; here, the cut-off function should equal one on the disk which is concentric to $D$ but has radius $u_{1} / 4$ and it should vanish near the boundary of the radius $u_{1} / 2$ 
disk. Furthermore, its derivatives should be bounded by $\zeta / u_{1}$. Finally, note that the $L_{1}^{2}$ norm of this function $u$ over $G$ here can then be bounded apriori using (A.42).

Step 5 The purpose of this step is to offer a proof of Lemma A.9:

Proof of Lemma A.9 As remarked previously, the number $\lambda_{n}$ which appears in (A.29) should be given as in (A.38).

To control the convergence of the sequence $\left\{\underline{\eta}_{j, n}\right\}$ as maps into $\mathrm{Sym}^{p} \mathbb{C}$, it proves useful to digress for a moment to consider the standard parametrization of the latter as $\mathbb{C}^{p}$. Here, $a=\left(a_{1}, \ldots, a_{p}\right) \in \mathbb{C}^{p}$ determines the $p$ 'th order, monic polynomial

$$
\mathcal{P}_{a}(\phi)=\phi^{p}+a_{1} \phi^{p-1}+\ldots+a_{p}
$$

and the roots of the latter give the corresponding point in $\operatorname{Sym}^{p} \mathbb{C}$. Conversely, a point $\gamma=\left\{\gamma_{j}\right\}_{1 \leq j \leq p} \in \operatorname{Sym}^{p} \mathbb{C}$, determines the polynomial $\mathcal{P}_{a}$, where the coefficients are given by

$$
a_{m}=\sum_{K \in \Theta(m)} \prod_{j \in K} \gamma_{j}
$$

Here the sum runs over the set $\Theta(m)$ of $m$-element subsets of $\{1, \ldots, p\}$.

Now consider the case where the coefficients in (A.52) are functions on $D(0)$ given by setting $\gamma_{j} \equiv \underline{\eta}_{j, m}$ in (A.53). Denote these functions by $\left\{a_{m, n}\right\}_{1 \leq m \leq p}$. (In this regard, note that $a_{1, n} \equiv 0$.) The fourth point of Lemma A.12 implies that for each $m \in\{1, \ldots, p\}$, the resulting sequence $\left\{a_{m, n}\right\}$ is bounded as $n$ tends to $\infty$ and satisfies a uniform bound on its Holder norm over $D(2)$. Moreover, because of the first point of Lemma A.12, this same sequence enjoys a uniform bound on its Sobolev $L_{1}^{2}$ norm.

The existence of the uniform $L_{1}^{2}$ bound implies that any infinite subsequence of the sequence $\left\{a_{m, n}\right\}$ has, itself, an infinite subsequence which converges weakly in $L_{1}^{2}$ over the half disk $D(0)$ to an $L_{1}^{2}$ function, $a_{m, \infty}$. By taking a so-called diagonal subsequence, one can arrange that the same subsequence converges for each of the possible values for $m$.

Note that the existence of the uniform Holder bound over $D(2)$ for each sequence $\left\{a_{m, n}\right\}$ implies that these subsequences converge strongly in the $C^{0}$ topology on $D(2)$ to give Holder continuous functions on $D(2)$. With the preceding understood, it follows that the limiting data $\left\{a_{m, \infty}\right\}_{1 \leq m \leq p}$ defines a Holder continuous and Sobolev class $L_{1}^{2}$ map from $D(2)$ into $\mathrm{Sym}^{p} \mathbb{C}$.

Given the preceding, the proof of Lemma A.9 will be complete with a demonstration that there exists $m \in\{1, \ldots, p\}$ and an infinite subsequence of $\left\{a_{m, n}\right\}$ whose limit, $a_{m, \infty}$, is not identically zero. This demonstration requires the following crucial lemma:

Lemma A.13 There exists a constant $c$ and an infinite subset $\Omega \subset\{1, \ldots\}$ with the following property: If $n \in \Omega$, then $0<\lambda_{n} \leq c \lambda_{n+4}$.

The proof of this lemma is provided in Steps 8-12. Assume its validity for now. 
Given Lemma A.13, here is how to complete the proof of Lemma A.9: To begin, take $a_{m, \infty}$ to be a limit of the sequence $\left\{a_{m, n}\right\}_{n \in \Omega}$. Now, suppose that $a_{m, \infty} \equiv 0$ for each $m$. This assumption generates a contradiction. Indeed, were all $a_{m, \infty}$ identically zero, then the sequence $\left\{\sum_{j}\left|\underline{\eta}_{j, n}\right|^{2}\right\}_{n=1,2, \ldots}$ would have a infinite subsequence which converged to zero uniformly over $D(2)$. However, if for some large $n, \sum_{j}\left|\underline{\eta}_{j, n}\right|^{2}$ is small on $D(2)$, say less than $\varepsilon$ everywhere, then, because of the second point of Lemma A.12,

$$
\int_{D(4)} \sum_{j}\left(\left|d \underline{\eta}_{j, n}\right|^{2}+u^{-2}\left|\operatorname{im}\left(\underline{\eta}_{j, n}\right)\right|^{2} \leq \zeta \cdot \varepsilon\right.
$$

where $\zeta$ is independent of both $n$ and $\varepsilon$. But, if (A.54) is true, then Lemma A.13 forces $\int_{D(0)} \sum_{j}\left(\left|d \underline{\eta}_{j, n}\right|^{2}+u^{-2}\left|\operatorname{im}\left(\underline{\eta}_{j, n}\right)\right|^{2}\right) \leq c \cdot \zeta \cdot \varepsilon$. And, this last condition contradicts the first point of Lemma A.12 when $\varepsilon$ is small.

Step 6 The purpose of this step and Step 7 is to present the proof of Lemma A.10.

Proof of Lemma A.10 Define a map, $q: D(2) \rightarrow\{1, \ldots, p\}$ by assigning to $w \in D(2)$ the number of distinct roots of $\mathcal{P}^{w}(\lambda) \equiv \phi^{p}+a_{1, \infty}(w) \phi^{p-1}+\ldots+a_{p, \infty}(w)$. Say that $w$ is a regular point of $D(2)$ when $q(w)$ maximizes the function $q(\cdot)$ on some neighborhood of $w$. Since $q$ maps into a finite set, it follows that the set of regular values in $D(2)$ is both open and dense. Note that on some neighborhood of a regular value, the roots, $\left\{\underline{\eta}_{j, \infty}\right\}$, of the polynomial $\mathcal{P}^{(\cdot)}$ can be viewed as a set of $p$ honest, complex valued functions. In fact, it follows from Lemma A.9 that each such $\underline{\eta}_{j, \infty}$ is Holder continuous and Sobolev class $L_{1}^{2}$ near a regular value point.

Now, let $w \in D(2)$ be a regular point. The claim here is that each $\left\{\underline{\eta}_{j, \infty}(w)\right\}$ obeys the equation

$$
\bar{\partial} \underline{\eta}_{j, \infty}-\frac{3}{2} u^{-1} \operatorname{im}\left(\underline{\eta}_{j, \infty}\right)=0
$$

on some neighborhood of a regular point in $D(2)$. This claim will be proved in Step 7, below, so accept it for now.

The proof of Lemma A.10 is completed as follows: Let $w \in D(2)$ be a regular point. Then, on some open neighborhood $U$ of $w$ which consists solely of regular points, the function $\tau$ is the product of those elements in $\left\{\underline{\eta}_{j, \infty}\right\}$ which are not identically zero on $U$. This identifies $\tau$ on a neighborhood of $w$ with one of the function $a_{m, \infty}$. Indeed, $\left.\tau\right|_{U}=a_{m, \infty}$ where $m$ is the smallest of the integers $m^{\prime} \in\{2, \ldots, p\}$ for which $a_{m^{\prime}, \infty}$ is not identically zero on $U$. Note that $\tau$ is continuous since it extends continuously as zero to the complement of the set of regular points. Furthermore, one can prove that $\tau$ is Sobolev class $L_{1}^{2}$ with

$$
\int_{D(2)}|d \tau|^{2} \leq \zeta \int_{D(2)} \sum_{1 \leq m \leq p}\left|d a_{m, \infty}\right|^{2} .
$$

(To prove (A.56), it is sufficient to exhibit a sequence of $L_{1}^{2}$ functions $\left\{\tau_{k}\right\}_{k=1,2, \ldots}$ which converges to $\tau$ in $L^{2}$ and such that each $\tau_{k}$ has $L_{1}^{2}$ norm bounded by the right 
side of (A.56) for some $k$ independent constant $\zeta$. To construct such a sequence, let $\chi$ denote the standard bump function on $[0, \infty)$ and set $\tau_{k} \equiv \chi(|\ln | \tau \mid / k) \cdot \tau$. Thus, $\left|\tau_{k}\right| \leq|\tau|$ and when $k$ is large, then $\tau_{k}=\tau$ where $|\tau| \geq e^{-k}$ and $\tau_{k}=0$ where $|\tau| \leq e^{-2 k}$. Thus, $\left\{\tau_{k}\right\}$ converges to $\tau$ in the $L^{2}$ topology. Furthermore, $\left|d \tau_{k}\right|=0$ where $|\tau| \leq e^{-2 k}$, and $\left|d \tau_{k}\right| \leq|d \tau|(1+\zeta / k)$ otherwise. This last inequality implies the $\tau_{k}$ version of (A.55) since $|d \tau| \leq \sum_{m}\left|d a_{m, \infty}\right|$ where $|\tau|>0$.) Next, remark that (A.55) implies that $\tau$ obeys (A.30) on the open set of regular points with the proviso that the integer $p^{\prime}$ and the function $\mathcal{P}$, may not extend continuously from this set to the whole of $D(2)$. In any event, define a function $f$ on $D(2)$ by setting $f=0$ at non regular points, and by setting $f=\frac{3 i}{4 u} p^{\prime}(1-\mathcal{P})$ on the set of regular points. Thus, $f$ is bounded, and measurable on any subset of $D(2)$ where $u$ is bounded away from zero.

Now, let $D \subset D(2)$ be a disk on which $u$ is bounded away from zero, and let $\kappa$ be a continuous and Sobolev class $L_{1}^{2}$ solution to the equation $\bar{\partial} \kappa=-f$ on $D$. Since $f$ is bounded and measurable on $D$, a standard construction with the kernel $\pi^{-1}\left(w-w^{\prime}\right)^{-1}$ for the operator $\bar{\partial}^{-1}$ will give a continuous and $L_{1}^{2}$ function on $D$ which solves this last equation.

With $\kappa$ understood, observe that $\tau=e^{\kappa} \tau_{0}$ on $D$, where $\tau_{0}$ is a continuous and Sobolev class $L_{1}^{2}$ function on $D$ which obeys the equation $\bar{\partial} \tau_{0}=0$ at all points where $\tau_{0} \neq 0$. Since $\tau_{0}$ is Sobolev class $L_{1}^{2}$, this last condition implies that $\tau_{0}$ is holomorphic everywhere on $D$. Thus, $\tau_{0}$ vanishes at no more than a finite set of points on any compact domain in $D$. And, since the zeros of $\tau_{0}$ are the same as those of $\tau$ in $D$, it follows that all but finitely many points of $D$ are regular points. In particular, this implies that the integer $p^{\prime}$ is in (A.30) is constant over $D(2)$ and that the function $\mathcal{P}$ is continuous on the complement of a set which is at worst countable, and which has no accumulation points where $u>0$.

Step 7 This step completes the proof of Lemma A.10 with a proof of (A.56). To begin the argument, let $w$ denote the regular point in question. By definition, the polynomial $\mathcal{P}^{w}$ has $q \equiv q(w)$ distinct roots, and it has this same number at all points $w^{\prime}$ which are close to $w$. Each root of $\mathcal{P}^{w}$ also has a multiplicity and this multiplicity is also constant on some neighborhood of $w$. With the preceding understood, let $\underline{\eta}_{\infty}$ be a root of $\mathcal{P}^{(\cdot)}$ thought of as a function, $\mathrm{k}$ on a neighborhood, $D$, of $w$. Let $e$ denote the multiplicity of this root. Then, for large $n$ which labels Lemma A.9's convergent subsequence, there will be precisely e elements of $\left\{\underline{\eta}_{j, n}\right\}$ whose values at points in $D$ are very close to the corresponding values of $\underline{\eta}_{\infty}$. Furthermore, all other elements in $\left\{\underline{\eta}_{j, n}\right\}$ will have values at the points of $D$ which differ substantially from the corresponding values of $\underline{\eta}_{\infty^{\prime}}$. In particular, the $e$ elements of $\left\{\underline{\eta}_{j, n}\right\}$ which are close to $\underline{\eta}$ form an unambiguous subset $\left\{\underline{\eta}_{j, n}\right\}$ over $D$ which one can declare, unambiguously, consists of the first $e$ elements of $\left\{\underline{\eta}_{j, n}\right\}$. Then, $\gamma_{n} \equiv \sum_{1 \leq j \leq e} \underline{\eta}_{j, n}$ defines an unambiguous, complex valued function on $\bar{D}$.

It then follows from (A.39) that the function $\gamma_{n}$ obeys the equation

$$
\bar{\partial} \gamma_{n}-\frac{3}{2} u^{-1} \operatorname{im}\left(\gamma_{n}\right)+\sigma_{j, n} \cdot \partial \gamma_{n}+\sum_{1 \leq j \leq e} \sum_{k} \sigma_{j, n}^{k} \cdot \operatorname{im}\left(\underline{\eta}_{k, n}\right)=0
$$


It then follows from the fourth and fifth assertions of Lemma A.12 that the last two terms on the right side of (A.57) converge to zero (in the $L^{2}$ topology) as $n$ tends to infinity, and thus (A.55) holds.

Step 8 This step begins the proof of Lemma A.13.

Proof of Lemma A.13 The fact that $\lambda_{n}>0$ follows from the assumption that the $\left\{\eta_{j}\right\}$ are not identical over the region $A$. Meanwhile, the argument used below to find the set $\Omega$ and the constant $c$, is a modified version of the argument used by Aronszajn in the proof of his unique continuation theorem in [1].

The argument given below is a proof by contradiction, so for this purpose. assume at the outset that there is no constant $c$ which makes Lemma A.13 true. This means that for any $c>0$, the numbers $\left\{\lambda_{n}\right\}$, though not identically zero, obey the inequality $\lambda_{n} \geq c \cdot \lambda_{n+4}$ when $n$ is sufficiently large. In particular, this last inequality implies that for any $c>0$ and sufficiently large $n$,

$$
0<\lambda_{n} \leq c^{-n}
$$

A contradiction will be shown to follow from this assumption.

Step 9 The contradiction to the assumption in (A.58) is derived with the help of (A.2) which is obeyed by each $\eta \in\left\{\eta_{j}\right\}$. The first remark with regard to (A.2) is that the term with $\alpha_{1}$ in (A.2) complicates the arguments that follow. The approach taken here for dealing with this term involves the replacement of the coordinate $w=t+i \cdot u$ by a new complex coordinate, $\varpi$. This particular step serves as a digression to introduce this new coordinate.

In particular, the coordinate $\varpi$ is chosen to have the following properties:

- $|\varpi-w| \leq \zeta \cdot|w|^{2}$ where $\zeta$ is independent of $w$ and $\delta$.

- $|\partial \varpi-1| \leq \zeta \cdot|w|$ where $\zeta$ is independent of $w$ and $\delta$ when $\delta$ is small.

- $\operatorname{Im}(\varpi)=0$ when $u=0$.

- $\bar{\partial} \varpi+\alpha_{1}(\eta) \cdot \partial \varpi=0$ where $\alpha_{1}$ appears in (A.2) and where

$$
\underline{\eta} \equiv \bar{p}^{-1} \cdot \sum_{j} \eta_{j}
$$

The point of introducing $\varpi$ is as follows: When $\eta$ in (A.2) is written in terms of $\varpi$, then the latter equation is equivalent to

$$
\begin{aligned}
& \bar{\partial}_{\varpi} \eta-\frac{3}{2} \operatorname{Im}(\varpi)^{-1} \cdot\left(1+\alpha_{3}\right) \cdot b \\
& \quad+\left(1-\alpha_{1}^{2}\right)^{-1} \cdot(\overline{\partial \varpi})^{-1} \cdot\left(\alpha_{1}(\eta)-\alpha_{1}(\underline{\eta})\right) \cdot\left(\partial_{w} \eta\right)=0,
\end{aligned}
$$

where $\alpha_{3}=-1+\left(1+\alpha_{2}\right) \cdot\left(1-\alpha_{1}^{2}\right)^{-1} \cdot(\overline{\partial \varpi})^{-1} \cdot \operatorname{im}(\omega) / b$ with $\alpha_{1}$ and $\alpha_{2}$ as in (A.2). (Here, $w, b, \partial_{w} \eta$ and $\partial \varpi$ are considered as functions of $\varpi$ by inverting the map $w \rightarrow \varpi(w)$.) Note that $\alpha_{3}$ obeys

$$
\left|\alpha_{3}\right| \leq c^{\prime} \cdot|\varpi|
$$


for some constant $c^{\prime}$ whichis independent of $\varpi$.

Here is an existence proof for the complex function $\varpi$ : To begin, reintroduce the bump function $\chi$ and then, with $\delta>0$ chosen to define the set $A$ as in Step 1 of the first section of this Appendix, set $\chi_{1}$ to denote the function on $\mathbb{C}$ given by $\chi(2|w| / \delta)$. Next, introduce $\alpha \equiv \alpha_{1}(\underline{\eta})$. Then, consider writing $\varpi=w \cdot e^{\sigma}$, where $\sigma$ is constrained to obey the equation

$$
\bar{\partial} \sigma+\chi_{1} \cdot w^{-1} \cdot(\alpha \cdot \partial \sigma+\alpha)=0 .
$$

(Note that $|\alpha| \leq \zeta \cdot|w|^{2}$ by virtue of (8.4) and (A.1). Thus, the coefficients in (A.62) are bounded.)

To be more precise, use the Greens kernel for the operator $\bar{\partial}$ to find a solution to (A.62) as a fixed point of the mapping $T$ which sends a complex valued function $\sigma$ to

$$
\begin{aligned}
& \pi^{-1} \cdot w \cdot \int_{u \geq 0} d^{2} \lambda\left[\left(\chi_{1} \cdot(\alpha \cdot \partial \sigma+\alpha) \cdot \lambda^{-2}(\lambda-w)^{-1}\right.\right. \\
& \left.+\left(\chi_{1} \cdot(\alpha \bar{\partial} \sigma+\alpha) \cdot \bar{\lambda}^{-2}(\bar{\lambda}-w)^{-1}\right)\right] .
\end{aligned}
$$

Note that $\bar{\partial} T(\sigma)=-\chi_{1} \cdot w^{-1} \cdot(\alpha \cdot \partial \sigma+\alpha)$ where $u>0$ so that $\varpi=w \cdot e^{\sigma}$ satisfies the fourth point in (A.59) when $\sigma$ is a fixed point of $T$. Also, $T(\sigma)$ is real where $u=0$ so that $\varpi$ will also satisfy the third point of (A.59). Thus, it remains only to find a fixed point $\sigma$ to (A.63) for which the corresponding function $\varpi$ obeys the first and second points of (A.59).

The contraction mapping theorem will be used to find a fixed point of $T$ with the required properties. In this regard, introduce the Banach space $\mathcal{H}$ which is defined by completing the compactly supported functions on $\mathbb{C}$ with the norm $\|\cdot\|_{*}$ whose square sends $\sigma$ to

$$
\sup _{\mathbb{C}}|\sigma|^{2}+\sup _{\mathbb{C}} \delta^{2}|d \sigma|^{2}+\sup _{0<r<2 d} \sup _{w \in \mathbb{C}}(\delta / r)^{c} \cdot \delta^{2} \cdot \int_{|\lambda-w| \leq r}|\nabla d \sigma|^{2} d^{2} \lambda .
$$

Here, $c \in(0,1)$ is half the value of the exponent $1 / \zeta$ which appears in $(8.12)$.

Since the map $T$ is linear inhomogeneous, the contraction mapping theorem will find a unique fixed point for $T$ on $\mathcal{H}$ given that

$$
\begin{aligned}
T(0) & \in \mathcal{H} . \\
\|T(\sigma)-T(0)\|_{*} & <2^{-1} \cdot\|\sigma\|_{*} .
\end{aligned}
$$

Moreover, to prove the first point in (A.65) it is sufficient to verify that $\|T(0)\|_{*}$ is finite; while the second point need be tested only with smooth functions having compact support.

Given that (A.65) holds then the contraction mapping theorem finds a fixed point $\sigma$ to $T$ with $\|\sigma\|_{*} \leq 2 \cdot\|T(0)\|_{*}$. Furthermore, if

$$
\|T(0)\|_{*} \leq \zeta \cdot \delta
$$


then it follows from the contraction mapping theorem that the fixed point $\sigma$ obeys $\sigma(0)=0$ and $|d \sigma| \leq \zeta$. These last conditions imply that $|\sigma| \leq \zeta \cdot|w|$ and thus the first two points in (A.59) will also be satisfied.

Both of the points in (A.65) and (A.66) can be addressed with the help of the following lemma:

Lemma A.14 Let $\tau$ be a continuous, Sobolev class $L_{1}^{2}$, complex valued function on $\mathbb{C}$ which vanishes where $u \leq 0$ and which has compact support on the disk of radius $\delta$ about the origin. Suppose that

$$
E^{2} \equiv \sup _{\mathbb{C}}|\tau|^{2}+\sup _{0<r \leq \delta} \sup _{w \in \mathbb{C}}(\delta / r)^{2 c} \int_{|\lambda-w| \leq r}|d \tau|^{2}
$$

is finite. Then,

$$
Q \equiv(4 \pi)^{-1} \cdot w \cdot \int_{u \geq 0}\left[\tau \cdot \lambda^{-1}(\lambda-w)^{-1}+\bar{\tau} \cdot \bar{\lambda}^{-1}(\bar{\lambda}-w)^{-1}\right] d^{2} \lambda
$$

is in $\mathcal{H}$ and satisfies $\|Q\|_{*} \leq \delta \cdot E$.

Note that Lemma A.14 with $\tau=\chi_{1} \cdot w^{-1} \alpha$ gives the first point in (A.68) for in this case, $E$ is bounded by $\zeta \cdot \delta^{c}$ by virtue of (A.1), (8.4) and (8.12). Indeed, as $\alpha=\zeta \cdot b^{2}+\mathcal{O}\left(b^{4}\right)$, the latter imply that $|\alpha| \leq \zeta \cdot|w|^{2}$ and $|d \alpha| \leq \zeta \cdot|w| \cdot \sum_{j}\left|d \nu_{j}\right|$. Thus, $|\tau|$ and $|d \tau|$ have support where $|w| \leq \delta$, while the former is bounded by $\zeta \cdot|w|$ and the latter is bounded by $\zeta \cdot\left(1+\sum_{j}\left|d \nu_{j}\right|\right)$. In particular, these last remarks with Lemma A.14 imply that $\|T(0)\|_{*} \leq \zeta \cdot \delta^{1+c}$ for some $\delta$ independent constant $\zeta$. This gives (A.66) for small $\delta$.

Meanwhile, Lemma A.14 with $\tau=\chi_{1} \cdot w^{-1} \cdot \alpha \cdot d \sigma$ gives the second point in (A.65) (for small $\delta$ ). In this case, $|\tau| \leq \zeta \cdot \delta \cdot|d \sigma|$ while $|d \tau| \leq \zeta \cdot(|d \sigma|+\delta \cdot|\nabla d \sigma|$ ) and both have support in the radius $\delta$ disk on $\mathbb{C}$ with center the origin. Thus, $E \leq \zeta \cdot \delta \cdot\|\sigma\|_{*}$ in this case.

Thus, the verification of (A.59) requires only the proof of Lemma A.14.

Proof of Lemma A.14 To begin, use the definition of $Q$ as an integral to find the pointwise bound $|Q| \leq \zeta \cdot \delta \cdot \sup _{\mathbb{C}}|\tau|$. Note also that where $|w|>4 \delta$, the definition of $Q$ gives $|d Q|+\delta \cdot|\nabla d Q| \leq \zeta \cdot\left(\sup _{\mathbb{C}}|\tau|\right) \cdot \delta^{2} /|w|^{2}$.

To estimate the last two terms in (A.64) where $|w| \leq 4 \cdot \delta$, it proves useful to employ the equation $\bar{\partial} Q=-\tau$ which is valid where $u>0$. Where $u \leq 0, \bar{\partial} Q$ is equal to the function $\tau^{\prime}$ who value at $t+i u$ is that of $-\bar{\tau}$ at the conjugate point $t-i \cdot u$. Differentiating these equations finds that $\bar{\partial}(d Q)=-d \tau$ where $u>0$ and $\bar{\partial}(d Q)=-d \tau^{\prime}$ where $u<0$. Note that there is no "delta function" mass for $\bar{\partial}(d Q)$ along $u=0$ because $\tau$ is zero there by assumption. With this last equation for $\bar{\partial}(d Q)$ understood, a bound on $\int_{|\lambda-w| \leq r}|\nabla d Q|^{2} d^{2} \lambda$ by $\zeta \cdot(\delta / r)^{2 c} \cdot \int_{|\lambda-w| \leq r}|d \tau|^{2} d^{2} \lambda$ follows using Morrey's Theorem 5.4.1 from [15]. Note that this same bound plus the aforementioned bound on $|d Q|$ at points where $|w| \geq 4 \delta$ implies the bound $|\nabla Q| \leq \zeta \cdot E$ at all points. Indeed, 
in this regard, one need only appeal to Theorem 3.5.2 in [15]. (Note that the bound for $|d Q|$ can be obtained directly from the integral equation for $Q$.)

Step 10 With the function $\underline{\eta}=p^{-1} \sum_{j} \eta_{j}$ understood, now re-introduce the difference functions $\underline{\eta}_{j}=\eta_{j}-\underline{\eta}$.

Remark first that (A.60) implies that the $\left\{\underline{\eta}_{j}\right\}$ obey an equation which has the schematic form

$$
\bar{\partial}_{\varpi} \underline{\eta}_{j}-\frac{3}{2} \operatorname{im}(\varpi)^{-1} \operatorname{im}\left(\underline{\eta}_{j}\right)+\sum_{k} R_{j k} \underline{\eta}_{k}=0,
$$

where $\left|R_{j k}\right| \leq \zeta \cdot \sum_{j}\left(\left|b_{j}\right|+\left|b_{j}\right| \cdot\left|d \eta_{j}\right|\right) \leq \zeta \operatorname{im}(\varpi)\left(1+\sum_{j}\left|d \eta_{j}\right|^{2}\right)$. Here, the 1-form norm can be taken using the Euclidean metric from the $\varpi$-plane. (This is equivalent to the Euclidean metric from the $w$-plane.)

To make use of this last equation, introduce the polar coordinates $(r, \theta)$ on the $\varpi-$ plane by writing $\varpi=r e^{i \theta}$. Thus, $2 \bar{\partial}_{\varpi}=e^{i \theta}\left(\partial_{r}+i \cdot r^{-1} \cdot \partial_{\theta}\right)$. In particular, (A.69) is equivalent to

$$
\partial_{r} \underline{\eta}_{j}+i r^{-1} \partial_{\theta} \underline{\eta}_{j}+i \frac{3}{2} r^{-1}(\cot \theta-i)\left(\underline{\eta}_{j}-\underline{\eta}_{j}\right)=-2 e^{-i \theta} \sum_{k} R_{j k} \underline{\eta}_{k} .
$$

Now, write $\underline{\eta}_{j} \equiv r^{-3 / 2} \sin ^{-3 / 2} \theta \alpha_{j}$ in which case (A.70) is equivalent to

$$
\partial_{r} \sigma_{j}+i r^{-1} \partial_{\theta} \sigma_{j}-i \frac{3}{2} r^{-1}(\cot \theta-i) \bar{\sigma}_{j}=-2 e^{-i \theta} \sum_{k} R_{j k} \sigma_{k} .
$$

To proceed with (A.71), fix $\varepsilon \in(0, \delta / 100)$ and a positive integer $m$ and then introduce the bump function $\chi_{\varepsilon, m}$ whose value at $\omega$ is $\chi(r / \varepsilon)\left[1-\chi\left(2^{m+1} r / \delta\right)\right]$. This function equals 1 where $2^{-m} \delta \leq r \leq \varepsilon$ and it vanishes where $r \geq 2 \cdot \varepsilon$ and where $r \leq 2^{-m-1} \delta$. Next, introduce $f_{j} \equiv \chi_{\varepsilon, m} \varpi^{-N} \sigma_{j}$, and then multiply both sides of (A.69) by $\chi_{\varepsilon, m} \varpi^{-N}$ to obtain the following equation:

$$
\begin{aligned}
& \partial_{r} f_{j}+i r^{-1} \partial_{\theta} f_{j}-i \frac{3}{2} r^{-1}(\cot \theta-i) e^{2 i N \theta} \bar{f}_{j} \\
& =-2 e^{-i \theta}\left(\sum_{k} R_{j k} f_{k}-\left(\bar{\partial} \chi_{\varepsilon, m}\right) \varpi^{-N} \sigma_{j}\right) .
\end{aligned}
$$

This "master equation" for $f_{j}$ will be analyzed further below. However, during the subsequent discussion, keep in mind that

$$
\left|f_{j}\right|=\chi_{\varepsilon, m} r^{-N+3 / 2} \sin ^{3 / 2} \theta\left|\underline{\eta}_{j}\right| .
$$

Step 11 With (A.72) understood, square both sides, sum over the index $j$ and integrate the result over $A$. The left hand side of the resulting equality can be manipulated with an integration by parts to read:

$$
\int_{A} \sum_{j}\left(\left|\partial_{r} f_{j}\right|^{2}+r^{-2}\left|\partial_{\theta} f_{j}-i \frac{3}{2} r^{-1}(\cot \theta-i) e^{2 i N \theta} \bar{f}_{j}\right|^{2}\right) .
$$


(When driving (A.74), remember that $f_{j}$ has support only where $2^{-m-1} \delta \leq r \leq 2 \varepsilon$, and $f_{j}$ also vanishes at $\theta=0, \pi$. As with the integration by parts in the proof of Lemma 8.2, there are no anomalous boundary terms from the points in $\pi(\Lambda)$ here.)

Because of the form of the right hand side of (A.72), the integral in (A.74) is no greater than

$$
8 \int_{A}\left(\sum_{j, k}\left|R_{j, k}\right|^{2}\right)\left(\sum_{i}\left|f_{i}\right|^{2}\right)+2 \int_{A}\left|d \chi_{\varepsilon, m}\right|^{2} r^{-2 N}\left(\sum_{j}\left|\sigma_{j}\right|^{2}\right) .
$$

The task now is to manipulate the expressions in (A.74) and (A.75) to obtain a useful inequality. For this purpose, the task ahead is to replace (A.74) by smaller terms and (A.75) by larger terms.

The first step in this strategy concerns the left most integral on the right side of (A.74). Here is the key lemma for treating this term:

Lemma A.15 There exists $\zeta \geq 1$ which is independent of $N$, and $m$ and such that when $\varepsilon<\zeta$, then the left most integral in (A.75) is no greater than $1 / 1000$ of the integral in (A.74).

This lemma is proved in Step 14, below.

Step 12 To continue with the argument for Lemma A.13, observe that Lemma A.15 has the following consequence: Let $\gamma \equiv\left(\sum_{j}\left|f_{j}\right|^{2}\right)^{1 / 2}$. As long as $\varepsilon$ is less than $\zeta^{-1}$ from Lemma A.5, then

$$
\int_{A}\left|\partial_{r} \gamma\right|^{2} \leq \zeta \int_{A}\left|d \chi_{\varepsilon, m}\right|^{2} r^{-2 N}\left(\sum_{j}\left|\sigma_{j}\right|^{2}\right) .
$$

where $\zeta$ is independent of $m$ and $N$ and $\varepsilon$. (This follows because of the inequality $\left|\partial_{r} \gamma\right| \leq\left(\sum_{j}\left|\partial_{r} f_{j}\right|^{2}\right)^{1 / 2}$.) Moreover, because $\gamma$ has support where $r<2 \varepsilon$, the left hand side of (A.76) is no greater than

$$
\zeta^{-1} \varepsilon^{-2} \int_{A} \gamma^{2}=\zeta^{-1} \varepsilon^{-2} \int_{A} r^{-2 N} \sum_{j}\left|\sigma_{j}\right|^{2}
$$

where $\zeta \geq 1$ is independent of $\varepsilon, N$ and $m$.

Meanwhile, the right hand side of (A.76) has an integrand whose support (for large $m$ ) is located in two disjoint regions. The first region has $r \in[\varepsilon, 2 \varepsilon]$ and the contribution from this region to the right side of (A.76) is no greater than

$$
\zeta \varepsilon^{-2} \int_{A \cap\{r, \varepsilon \leq r \leq 2 \varepsilon} r^{-2 N} \sum_{j}\left|\sigma_{j}\right|^{2} .
$$

The other region that contributes to the right hand side of (A.76) has $r$ restricted to $\left[2^{-m-2} \delta, 2^{-m} \delta\right]$. The claim now is that the contribution to the right hand side of (A.76) from this second region tends to zero as $m$ is taken to infinity with $N$ and $\varepsilon$ 
fixed. To see that such is the case, first note that the contribution from the region in question is no greater than

$$
\zeta \cdot 2^{2(N+1) m} \delta^{-2(N+1)} \int_{A \cap\left\{r_{*} 2^{-m-2} \delta \leq r \leq 2^{-m} \delta\right\}} \sum_{j}\left|\underline{\eta}_{j}\right|^{2} r \sin ^{3} \theta d r d \theta .
$$

Now let $\kappa \equiv\left(\sum_{j}\left|\underline{\eta}_{j}\right|^{2}\right)^{1 / 2}$. Note that $\kappa$ vanishes at $r=0$. Thus, the fundamental theroem of calculus finds that for any constant $r^{\prime}$, one has

$$
\int_{0 \leq \theta \leq \pi}\left|\kappa\left(r^{\prime}, \theta\right)\right|^{2} \sin ^{3} \theta d \theta \leq \int_{0 \leq \theta \leq \pi}\left(\int_{0 \leq r \leq r^{\prime}} \partial_{r} \kappa d r\right)^{2} \sin ^{3} \theta d \theta .
$$

And, Holder's inequality asserts that the right hand side in (A.80) is no greater than

$$
\int_{A \cap\left\{r \leq r^{\prime}\right\}}|d \kappa|^{2} r \sin ^{3} \theta d r d \theta
$$

In particular, since $|d \kappa| \leq\left(\sum_{j}\left|d \underline{\eta}_{j}\right|^{2}\right)^{1 / 2}$, these last two inequalities imply that (A.79) is no greater than

$$
\zeta \cdot 2^{2(N+1) m} \delta^{-2(N+1)} \lambda_{m-1}^{2}
$$

when $m$ is large. This last expression tends to zero as $m$ tends to infinity because of the assumption that (A.58) holds for any $c$ if $n$ is sufficiently large.

Step 13 With (A.79) seen to vanish as $m$ tends to infinity, one obtains from (A.77) and (A.78) and Lemma A.15 the following conclusion: There exists $\zeta \geq 1$ such that when $\varepsilon \leq \zeta^{-1}$, then

$$
\begin{aligned}
& \int_{A \cap\{r \leq \varepsilon / 2\}} r^{-2 N} \sum_{j}\left|\underline{\eta}_{j}\right|^{2} r \sin ^{3} \theta d r d \theta \\
& \leq \zeta \int_{A \cap\{\varepsilon \leq r \leq 2 \varepsilon\}} r^{-2 N} \sum_{j}\left|\underline{\eta}_{j}\right|^{2} r \sin ^{3} \theta d r d \theta .
\end{aligned}
$$

This last inequality can be exploited to complete the proof of Lemma A.13 as follows: Multiply both sides of (A.83) by $(3 \varepsilon / 4)^{2 N}$ to find that

$$
\begin{aligned}
& \int_{A \cap\{r \leq \varepsilon / 2\}} \sum_{j}\left|\eta_{j}\right|^{2} r \sin ^{3} \theta d r d \theta \\
& \leq \zeta \cdot(15 / 16)^{N} \cdot \int_{A \cap\{r \leq \varepsilon\}} \sum_{j}\left|\eta_{j}\right|^{2} r \sin ^{3} \theta d r d \theta .
\end{aligned}
$$

Since $N$ here can be arbitrarily large, it follows that all $\eta_{j} \equiv 0$. As this is assumed not to be the case, Lemma A.13 is proved.

Step 14 This step contains the proof of Lemma A.15. 
Proof of Lemma A.15 To begin the argument, recall that $\left|R_{j, k}\right| \leq \zeta r \sin \theta(1+$ $\left.\sum_{j}\left|d \eta_{j}\right|\right)$, and thus the left most term in (A.75) is no greater than

$$
\zeta \int_{A} r^{2} \sin ^{2} \theta\left(\sum_{j}\left|f_{j}\right|^{2}\right)+\zeta \int_{A} \sum_{j} r^{2} \sin ^{2} \theta\left(\sum_{j}\left|d \eta_{j}\right|^{2}\right)\left(\sum_{j}\left|f_{j}\right|^{2}\right)
$$

To deal with the left most term in (A.85), reintroduce $\gamma \equiv\left(\sum_{j}\left|f_{j}\right|^{2}\right)^{1 / 2}$. As previously remarked, $\left|\partial_{r} \gamma\right|^{2} \leq \sum_{j}\left|\partial_{r} f_{j}\right|^{2}$ and $\int_{A}\left|\partial_{r} \gamma\right|^{2} \geq \zeta^{-1} \varepsilon^{-2} \int_{A} \gamma^{2}$. Thus,

$$
\int_{A} \sum_{j}\left|f_{j}\right|^{2} \leq \zeta \varepsilon^{2} \int_{A} \sum_{j}\left|\partial_{r} f_{j}\right|^{2}
$$

where $\zeta$ is independent of $\varepsilon, m$ and $N$. Thus, the left most term in (A.85) is no greater than

$$
\zeta \varepsilon^{4} \int_{A} \sum_{j}\left(\left|\partial_{r} f_{j}\right|^{2}+r^{-2}\left|\partial_{\theta} f_{j}-i \frac{3}{2} r^{-1}(\cot \theta-i) e^{2 i N \theta} \bar{f}_{j}\right|^{2}\right)
$$

where $\zeta$ is independent of $\varepsilon, m$ and $N$.

The strategy for bounding the right most term in (A.85) calls on Lemma 8.2 and Lemma 5.4.1 in [15]. However, certain manipulations must be done first. In this regard, the first step is to produce some special function partitions for $(0, \pi)$ and also for $(0,2 \varepsilon)$.

With regard to the interval $(0, \pi)$, introduce, for each $k \in\{0,1, \ldots\}$, the angle $\theta_{k}=$ $2^{-k}(\pi / 2)$. Then, let $\chi$ denote the standard bump function which is zero on $[2, \infty)$ and 1 on $[0,1])$. However, here, assume $\chi \equiv 0$ on $[3 / 2, \infty)$. Now, set $\chi_{k}^{\theta}(\theta) \equiv$ $\chi\left(\left|\theta-\theta_{k}\right| / \theta_{k+1}\right)$. Thus, $\chi_{k}^{\theta}=0$ if $\theta<\theta_{k} / 4$ or if $\theta>7 \theta_{k} / 4$. On the other hand, $\chi_{k}^{\theta}=1$ if $\theta_{k} / 2 \leq \theta \leq 3 \theta_{k} / 2$. (Note that $\theta_{k} / 2=\theta_{k+1}$.) With the preceding understood, set $\theta_{k}$ for $k \in\{\ldots,-2,-1\}$ to equal $\pi-\theta_{-k}$, and define $\chi_{k}^{\theta}(\theta)$ by reflecting $\chi_{-k}^{\theta}$ through the point $\pi / 2$. Thus defined, the set $\left\{\chi_{k}^{\theta}\right\}_{k \in \mathbb{Z}}$ defines a partition of $(0, \pi)$ with a uniform bound on the number of elements which are non-zero at any one point, but with at least one element equal to one at each point.

As for the interval $(0,2 \varepsilon)$, introduce $r_{k} \equiv 2^{-k+1} \varepsilon$, and set $\chi_{k}^{r}(r) \equiv \chi\left(\left|r-r_{k}\right| / r_{k+1}\right)$. The set $\left\{\chi_{k}^{r}\right\}_{k \geq 0}$ defines a partition of $(0,2 \varepsilon)$ with a uniform bound on the number of elements which are non-zero at each point, but with at least one element equal to one at each point.

With the preceding understood, it follows that

$$
\sum_{j}\left|f_{j}\right|^{2} \leq \sum_{k, k^{\prime}}\left|\chi_{k}^{\theta} \chi_{k^{\prime}}^{r} f_{j}\right|^{2} .
$$

Thus, the second term in (A.85) is no greater than

$$
\zeta \sum_{k, k^{\prime}} r_{k^{\prime}}^{2} \sin ^{2} \theta_{k} \int_{A}\left(\sum_{j}\left|d \eta_{j}\right|^{2}\right) \gamma_{k, k^{\prime}}^{2},
$$

Geometry $8 \mathcal{G}$ Topology, Volume 2 (1998) 
where $\gamma_{k, k^{\prime}} \equiv\left(\sum_{j}\left|\chi_{k}^{\theta} \chi_{k^{\prime}}^{r} f_{j}\right|^{2}\right)^{1 / 2}$.

Now, use Lemma 8.2 and Lemma 5.4.1 in [15] on each $\left(k, k^{\prime}\right)$ term in (A.89) to find a constant $\zeta \geq 1$ which is independent of $k, k^{\prime}, N, m$ and $\varepsilon$ and is such that (A.89) is no greater than

$$
\zeta \sum_{k, k^{\prime}} r_{k^{\prime}}^{2+1 / \zeta} \sin ^{2+1 / \zeta} \theta_{k} \int_{A}\left|d \gamma_{k, k^{\prime}}\right|^{2} .
$$

Then, differentiate $\gamma_{k, k^{\prime}}$ to find that this last expression is no greater than

$$
\begin{aligned}
& \zeta \sum_{k, k^{\prime}} r_{k^{\prime}}^{2+1 / \zeta} \sin ^{2+1 / \zeta} \theta_{k}\left\{\int _ { A } \left(r_{k^{\prime}}^{-2} \theta_{k}^{-2}\left|\chi_{k}^{\prime \theta}\right|^{2} \chi_{k}^{r}\right.\right. \\
& \left.\left.+r_{k}^{-2} \chi_{k}^{\theta}\left|\chi_{k^{\prime}}^{\prime r}\right|^{2}\right) \sum_{j}\left|f_{j}\right|^{2}+\int_{A} \chi_{k}^{\theta} \chi_{k^{\prime}}^{r} \sum_{j}\left|d f_{j}\right|^{2}\right\} .
\end{aligned}
$$

Here, $\chi^{\prime}$ denotes the derivative of the function $\chi$.

Proceeding further, the triangle inequality applied to $\left|d f_{j}\right|^{2}$ finds the latter no less than

$$
\begin{aligned}
& \zeta\left(\left|\partial_{r} f_{j}\right|^{2}+r^{-2}\left|\partial_{\theta} f_{j}-i \frac{3}{2} r^{-1}(\cot \theta-i) e^{2 i N \theta} \bar{f}_{j}\right|^{2}\right. \\
& \left.+r_{k^{\prime}}^{2} \sin ^{-2} \theta_{k}\left|f_{j}\right|^{2}\right) .
\end{aligned}
$$

Together, (A.91) and (A.92) imply that the second term in (A.85) is no greater than

$$
\begin{aligned}
& \zeta\left(\sum_{k, k^{\prime}} r_{k^{\prime}}^{1 / \zeta} \sin ^{1 / \zeta} \theta_{k}\right)\left(\int_{A} \sum_{j}\left|\partial_{r} f_{j}\right|^{2}\right. \\
& \left.+r^{-2}\left|\partial_{\theta} f_{j}-i \frac{3}{2} r^{-1}(\cot \theta-i) e^{2 i N \theta} \bar{f}_{j}\right|^{2}+\int_{A} \sum_{j}\left|f_{j}\right|^{2}\right) .
\end{aligned}
$$

(Here, as in the previous lines, $\zeta$ is independent of $\varepsilon, m$ and $N$.) Since the double sum here is no greater than

$$
\zeta \varepsilon^{1 / \zeta}\left(\sum_{k=1,2, \ldots} 2^{-k / \zeta}\right)^{2} \leq \zeta^{\prime} \varepsilon^{1 / \zeta^{\prime}}
$$

the second term in (A.85) can not be greater than

$$
\begin{aligned}
& \zeta \varepsilon^{1 / \zeta} \int_{A} \sum_{j}\left(\left|\partial_{r} f_{j}\right|^{2}+r^{-2} \mid \partial_{\theta} f_{j}-\right. \\
& \left.\left.i \frac{3}{2} r^{-1}(\cot \theta-i) e^{2 i N \theta} \bar{f}_{j}\right|^{2}+\int_{A} \sum_{j}\left|f_{j}\right|^{2}\right) .
\end{aligned}
$$

Here, $\zeta$ is independent of $\varepsilon, m$ and $N$.

Together, (A.86), (A.87) and (A.95) imply the claim in Lemma A.15. 